\title{
Anomalous Diffusion in Ecology
}

\author{
Dissertation
}

zur Erlangung des mathematisch-naturwissenschaftlichen

Doktogrades

"Doctor rerum naturalium"

der Georg-August-Universität Göttingen

im Promotionsprogramm Physics of Biological and Complex Systems der Georg-August University School of Science (GAUSS)

vorgelegt von

\section{Mirko Luković}

aus Belgrad, Serbien

Göttingen 2013 


\section{Betreuungsausschuss}

Prof. Dr. Theo Geisel

Abteilung Nichtlineare Dynamik, Max-Planck-Institut für Dynamik und Selbstorganisation \& Georg-August-Universität Göttingen

Prof. Dr. Marc Timme

Abteilung Netzwerk-Dynamik, Max-Planck-Institut für Dynamik und Selbstorganisation

Prof. Dr. Fred Wolf

Abteilung Nichtlineare Dynamik, Max-Planck-Institut für Dynamik und Selbstorganisation

\section{Mitglieder der Prfungskommission}

\section{Referent: Prof. Dr. Theo Geisel}

Abteilung Nichtlineare Dynamik, Max-Planck-Institut für Dynamik und Selbstorganisation \& Georg-August-Universität Göttingen

Korreferent: Prof. Dr. Marc Timme

Abteilung Netzwerk-Dynamik, Max-Planck-Institut für Dynamik und Selbstorganisation

Prof. Dr. Fred Wolf

Abteilung Nichtlineare Dynamik, Max-Planck-Institut für Dynamik und Selbstorganisation

Prof. Dr. Ulrich Parlitz

Abteilung Biomedizinische Physik, Max-Planck-Institut für Dynamik und Selbstorganisation

\section{Dr. Eleni Katifori}

Abteilung Physik Biologischer Organisation, Max-Planck-Institut für Dynamik und Selbstorganisation

\section{Prof. Dr. Reiner Kree}

Institut für Theoretische Physik, Georg-August-Universität Göttingen

Tag der mündlichen Prüfung: 6. Februar, 2014. 
I hereby declare that this dissertation has been written independently and with no other sources or aids than those quoted.

Mirko Luković

Göttingen, December 2013. 


\section{Contents}

1 Introduction $\quad 5$

2 Anomalous Diffusion and Random Search Strategies 9

2.1 Anomalous Diffusion . . . . . . . . . . . . . . . . . . . 9

2.2 Random Search in Foraging . . . . . . . . . . . . . . . . . . . 10

2.2.1 An Idealised Model . . . . . . . . . . . . . . . . . . . . . . . . 11

2.2.2 Random Search on a Lattice . . . . . . . . . . . . . . . . 13

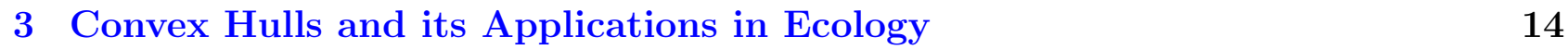

3.1 Convex Hulls of Planar Brownian Paths . . . . . . . . . . . . . . . . 16

3.2 Convex Hulls of Lévy Walks . . . . . . . . . . . . . . . . . . . . . . 35

3.3 Discriminating between normal and anomalous diffusion . . . . . . . . . 38

3.4 Discussion . . . . . . . . . . . . . . . . . . . 47

4 Convex Hulls of Continuous Time Random Walks $\quad 51$

4.1 Subordinated Brownian Motion . . . . . . . . . . . . . . . . 52

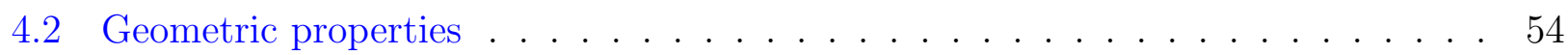

4.2 .1 Average perimeter . . . . . . . . . . . . . . 56

4.2 .2 Average Area . . . . . . . . . . . . . . . . . . 56

4.2 .3 Discussion . . . . . . . . . . . . . . . . . . . 59

4.3 Subordinated Lévy flights . . . . . . . . . . . . . . . . . . . . 59

4.4 Conclusion . . . . . . . . . . . . . . . . . . . . . 62

$\begin{array}{ll}\text { Summary \& Outlook } & 64\end{array}$

$\begin{array}{ll}\text { Bibliography } & 65\end{array}$ 


\section{Chapter 1}

\section{Introduction}

As measurement techniques improve and increasingly sophisticated analysis methods are more common, biology becomes subject to the wide range of treatments coming from physics. In this thesis, we consider a specific application of this trend, applying the theory of stochastic processes, and of random walks in particular, to the field of ecology. This chapter serves to introduce the main concepts and motivate the application. We end the chapter with organisational remarks.

\section{Movement Ecology}

Animals have to search for resources such as food, partners and shelter in order to reproduce and survive. The geographic area in which they do this is known as the home range. The home range is also the territory in which the animals perform their daily routines such as sleeping and socializing $[1,2]$.

Depending on the sensory and cognitive abilities of a foraging animal, the adopted search strategy can range from systematic to completely random. Strong cognitive abilities, such as a well developed memory, allow the animal to avoid visiting the same place more than once over a relevant period of time. Only in extreme cases, however, the animal can perform a purely systematic search. In some cases, animals may use cues available in their environment in order to restrict the territory to be searched. Depending on the nature of the cue, the animals can use their senses to move more directly towards the target. An example of such biased movement is chemotaxis [3]. Information or cues available to the animal might be weak or intermittent so that in some cases, the systematic search strategy would have to be replaced by a more random strategy.

At the other end of the spectrum are the purely random search strategies that neither rely on memory nor on information. In such cases the randomly moving forager can detect a target only from a short range, much shorter than the typical extension of the home range. This is the class of random searches that will be dealt with in this thesis. Considering the movement of the forager as random can be regarded as a first-order approximation or a limiting model of 
an animal with limited cognitive capabilities. It allows us to minimize the role of cognition and concentrate on the statistical properties of the movement [4].

\section{Stochastic Processes in Nature}

A theoretical approach to the study of animal foraging is beneficial because we do not consider a specific animal species, but rather the classes of tasks to be performed and their corresponding movement strategies. When considering random foraging strategies, the appropriate models are stochastic processes in the form of random walks and their extensions [5].

The simplest model of random foraging is a random walker moving on a plane in search of targets that are randomly distributed. A random walk on a plane is characterised by a sequence of independent jumps that are randomly sampled from a bivariate probability distribution of finite variance. The process is isotropic so that at every jump the searcher is equally likely to move in any direction. Finally, the jumps are also uncorrelated, meaning that the directions chosen are independent from the previous ones.

This simple model has been extended in many ways to better reflect specific characteristics of foraging animals such as the their tendency to move forward or towards the source of a particular cue [6]. The former is a form of persistence and it is enough to relax the assumption of independence in the random walk. The latter, on the other hand, is a global bias towards a particular direction and this type of movement can be modelled by assuming anisotropy.

Given that the jumps are sampled from a distribution with finite variance, the diffusive component of the corresponding mean square displacement will depend linearly on the number of jumps taken.

A further extension can be made by sampling the jump lengths randomly from a distribution that is broad-tailed with a diverging variance. Random walks constructed in such a way are know as Lévy flights and they have been used extensively to model transport phenomena that are faster then normal diffusion. They are characterised by a lack of scale and self-similar properties.

It has been proposed that under certain conditions, Lévy flights represent the optimal search strategy since they minimize the mean first passage time to a target. This proposal is known in the scientific community as the Lévy flight foraging hypothesis [4].

\section{Lévy Flight Foraging Hypothesis}

The idea that Lévy flights might give animals a slight evolutionary advantage over variants of the normal random walk while foraging in resource-scarce environments was first put forward by Shlesinger and Klafter in 1986 [7]. The probability of returning to a previously visited site is smaller than for a Gaussian distribution, thus avoiding oversampling. There is an ongoing debate within the scientific community as to whether there truly exist cases in nature where Lévy flights are used as the foraging strategy [8, 9]. 
One of the goals of the present thesis is to develop a method for determining whether a given movement pattern is truly Lévy-like. The standard method is based on identifying the turning points from the data and collecting the obtained jump lengths into a distribution, which, for example, is then subjected to a maximum likelihood test and an Akaike weight analysis. The test determines the likelihood that the distribution is a power law and therefore that the processes are Lévy-like $[10,11,12]$.

We take a new approach and propose the use of convex hulls (minimum convex polygon enclosing the recorded points [13]) of the animal home range. The method is robust and accurately discriminates between different foraging patterns. Moreover, the method is simple and robust even in the case where the data available is sparse. In addition, no prior knowledge of the temporal order of in which the animals visited the registered points is required. For the many foraging datasets that lack such information, the standard method cannot be applied, and therefore the method we propose is preferable.

\section{The Convex Hulls of Stochastic Processes}

In the often encountered two-dimensional environment it is desirable to quantify the geometric properties of the area covered by a random walker. A simple and widely employed approach makes use of the convex hull of the trajectory. In both experimental and theoretical ecology there is interest in the estimation of the geographic range over which single or groups of animals forage in order to better plan habitat conservation. Since the motion of many foraging animals is approximately random, the average area of the convex hull enclosing their trajectories can be used as a good estimate of the geographic range [14]. Other applications include determining the spatial extent of an epidemic outbreak among animals and potentially, outside of biology, assessing the area affected by spreading contaminants.

Convex hulls of Markovian processes such as Brownian motion have only recently been taken into consideration. In particular, previous methods are based on average properties such as the average perimeter and area. In this thesis we go beyond, by considering the distributions of these properties. Moreover, we also examine the geometric properties of more general Lévy-like and non-Markovian processes. 


\section{Organisation and Results}

The thesis is organised as follows:

Chapter 2 - Fundamentals of stochastic processes in Ecology: Through a selective review and replication of previous work, we motivate the study of anomalous diffusion in the context of ecology through the random search strategies of foraging animals. We introduce Lévy walks and the Lévy flight foraging hypothesis and show that there are conditions under which Lévy flights outperform normal random walks as a search strategy.

Chapter 3 - Convex hulls as indicators of foraging strategies: We introduce convex hulls and consider them as a model for the home range of foraging animals. We use numerical methods and scaling considerations to determine the properties of convex hulls of Lévy walks. Motivated by the ongoing debate regarding whether or not there exist animals that perform a Lévy walk, we propose a new robust method for discriminating between random walks and Lévy walks. We provide an intuitive explanation as to why the central limit theorem does not hold in the case of the probability distribution of the perimeter of a planar random walk.

Chapter 4 - Convex hulls of sub-diffusive processes: We discuss continuous time random walks and their role in ecology. We derive exact analytical expressions for the evolution of the average perimeter and area of the convex hull of this class of non-Markovian sub-diffusive processes. We also provide a method simpler than those available in the literature to determine the exact expression of the distribution of the maximum excursion of a continuous time random walks.

We conclude the thesis with a summary of the results and an outlook into future work. 


\section{Chapter 2}

\section{Anomalous Diffusion and Random Search Strategies}

\subsection{Anomalous Diffusion}

Anomalous diffusion is associated to a general class of random processes that do not follow Gaussian statistics. More formally, they are random processes that do not obey Fick's second law. They are characterised by a mean square displacement that does not depend linearly on time. This is usually due to the non-Markovian nature of the process or because of the lack of conditions necessary for the central limit theorem to hold true. Consequently, a general random walker with position $x(t)$ will have a mean square displacement that can often be expressed as a power law in the form $\left\langle x^{2}(t)\right\rangle \sim D_{\gamma} t^{\gamma}$, where $D_{\gamma}$ is the generalized diffusion with dimension $\left[D_{\gamma}\right]=\left[L^{2}\right] /\left[T^{\gamma}\right]$ and $0 \leq \gamma \leq 2$. Whenever $0 \leq \gamma<1$ the transport is typically slower than normal diffusion and therefore the term sub-diffusion is used. On the other hand, when $1<\gamma \leq 2$ one speaks of super-diffusion. Finally, when $\gamma=2$, the underlying process is said to be ballistic.

The simplest Markovian model that shows super-diffusive behaviour is the Lévy flight, a term coined by Mandelbrot in 1982 [15]. Lévy flights are simple random walks with jump lengths $l$ that are sampled from a probability distribution with a with a power law tail $p(l) \sim l^{-1-\alpha}$, where $0<\alpha<2$. A power law distribution is characterised by diverging variance for $0<\alpha<2$ and a diverging mean for $0<\alpha<1$. Such a heavy tailed distribution implies that the probability of having arbitrarily large jump lengths is significant, leaving the process without a properly defined scale and a mean square displacement that grows super-linearly with time.

The fact that the jumps lack a proper scale and occur instantaneously, renders the Lévy flight model unrealistic for most natural phenomena, particularly animal movement. We cannot assume that a forager is able to cover a broad scale of distances within the same time interval. The problem with the scale can be solved by introducing a cut-off in the jump distribution and creating what is known in the literature as the truncated Lévy flight. However, even truncated Lévy flights cannot overcome the problem of instantaneous jumps over long distances. The 
problem can be overcome by correlating the jump length with the travel time. One such model, introduced by Geisel et al. [16], is the Lévy walk, whereby a random walker moves with a constant velocity $\mathbf{v}$ between the turning points of its trajectory.

The Lévy walk is a specific type of random velocity model where the random travel times $\tau$ between two consecutive turning points are distributed according to an inverse power law. In that case the displacement between two turning points, which from here onwards we will refer to as the jump length (technically it is a run or flight), is given by $\lambda=\tau \mathbf{v}$. Consequently, the mean square displacement of the Lévy walk remains finite. Indeed, a snapshot in time of the trajectory of a Lévy walk will look very much like the trajectory of a Lévy flight.

\subsection{Random Search in Foraging}

The Lévy flight foraging hypothesis was first posed by Cole [17] in 1995, followed by an attempt to confirm it in a natural environment by Viswanathan et al. [18] in 1996. The argument is that because of oversampling, the normal planar random walk should be less efficient than a Lévy walk as a strategy for searching for resources. Whether there is compelling evidence in nature for this has been discussed controversially. Are there foraging animals in nature that perform Lévy walks rather than normal random walks in order to improve their chances of finding resources and hence improve their likelihood of survival? Since 1995 several examples of Lévy behaviour have been found in nature [4]. Ironically, the first example involving the wandering albatross was later revealed to be flawed [10].

The Lévy flight foraging hypothesis should be viewed in the context of a limiting model where the animals are assumed to move randomly on a plane with no internal states [4].

There are cases where strong evidence was found in favour of Lévy walks. For example Sims et al. found strong support for Lévy search patterns across 14 species of open-ocean predatory fish, with some individuals switching between Lévy and Brownian movement as they traversed different habitat types [19, 20]. Another example where Lévy walk patterns can be found in the work by Ramos-Fernández on the movement habits of spider monkeys [21].

Within the physics community, there are two main approaches to modelling animal movement and its efficiency. One approach, endorsed by Viswanathan et al. [4] is the use of Lévy walks as the most general form of random walk on a continuous plane with an agent that can detect a target that is within its visibility radius. The radius and the exponent $\alpha$ of the jump distribution of the Lévy walk are then used as the parameters in the optimisation problem.

On the other hand, Bénichou et al. [22] advocate the use of intermittent search strategies to model the movement of foraging animals. In such a scenario, there exist two alternating phases, the search/scanning phase and the motion/relocation phase. During the motion phase, targets cannot be detected. They show that there exists a global minimum for the mean search time $\langle t\rangle$ and that this can be achieved by selecting the correct distributions for the duration of each of the two phases. This model accounts for the degraded perception in animals due to 
velocity and it also takes into account situations where the target is hidden so that searching and moving become incompatible.

\subsubsection{An Idealised Model}

The first to notice the advantage of Lévy walks over simple random walks as a random search strategy were Shlesinger and Klafter [7]. The general argument was that the probability of returning to a previously visited site is smaller than for a Gaussian distribution and that the number of new visited sites is much larger for $N$ Lévy walkers than for $N$ Brownian walkers $[23,24]$. In other words, with Lévy walks oversampling of a territory is avoided.

This idea was formalised by Viswanathan et al. with a simple, idealized model that can account for the special role of $\alpha=1$ and for the fact that Lévy walks are optimal only when the target density is very low [25]. The model basically consists of a random searcher that moves on an Euclidean plane with randomly distributed targets. The searcher performs a Lévy walk on the plane with $0<\alpha \leq 2$, a fixed velocity $\boldsymbol{v}$ and a predefined detection radius $r_{v}$ within which a target can be seen. As soon as a target falls within the detection range, the searcher moves straight towards it, interrupting the Lévy walk. Once the target is reached, the searcher resumes the Lévy walk in search for other targets. The interruptions of the Lévy walk by the targets induce a cut-off in the jump distribution of the Lévy walk. As a result, the longest jumps that can be made during the Lévy walk are of the order of the mean free path $\lambda$ of the searcher between successive target encounters. The usual definition of the mean free path gives $\lambda=\left(2 r_{v} \rho\right)^{-1}$, where $\rho$ is the target density. Consequently, for high enough densities, i.e. when $\lambda \sim r_{v}$, the jump distribution will be heavily truncated such that its average value $\langle l\rangle$ becomes comparable to $\lambda$. Therefore, according to this model the random search efficiency in resourcerich environments does not depend on the parameter $\alpha$. However, when the target density is very low, $\langle l\rangle$ will strongly depend on $\alpha$. The authors of [25] define the search efficiency $\eta(\alpha)$ as the ratio between the number of sites visited and the total distance covered by the searcher, so that

$$
\eta(\alpha)=\frac{1}{\langle l\rangle \nu}
$$

where $\nu$ is the average number of jumps between two successive target sites. They argue that low values of $\eta$ are the result of high values of $\langle l\rangle$ (i.e., small $\alpha$ ) or because of high values of $\nu$ (i.e., large $\alpha$ ) and that it is therefore reasonable to conclude that there could be a maximum in $\eta$ for intermediate values of $\alpha$.

If we assume that the jump length distribution is given by $p(l) \sim l^{-1-\alpha}$, the mean jump length is give by

$$
\langle l\rangle \approx \frac{\int_{r_{v}}^{\lambda} l^{\alpha} \mathrm{d} l+\lambda \int_{\lambda}^{\infty} l^{-1-\alpha} \mathrm{d} l}{\int_{r_{v}}^{\infty} l^{-1-\alpha} \mathrm{d} l}=\frac{\alpha}{1-\alpha} r_{v}^{\alpha}\left(\lambda^{1-\alpha}-r_{v}^{1-\alpha}\right)+\lambda^{1-\alpha} r_{v}^{\alpha} .
$$

The targets can either be renewable so that they can be visited over and over again by the forager or they can be destructive in which case the available resources get depleted as the 
search progresses. Renewable targets include those that are either destroyed and then replaced or regrown in a relatively short amount of time or those that are not destroyed at all. Both cases yield very similar results [26]. Depending on whether the search is destructive or not, the average number of jumps necessary to achieve an average displacement $\lambda$ between successive target sites scales respectively as $\nu_{d} \approx\left(\lambda / r_{v}\right)^{\alpha}$ and $\nu_{n} \approx\left(\lambda / r_{v}\right)^{\alpha / 2}$ [25]. Therefore, by fixing the values of $r_{v}$ and $\rho$ it is possible to study the dependence of $\eta$ on $\alpha$ alone. The graphs of $\eta(\alpha)$ for the destructive and non-destructive case are shown in figure 2.1. It is only in the latter case that the graph is non-monotonic with a maximum value for at $\alpha \approx 1-\left[\ln \left(\lambda / r_{v}\right)\right]^{-1}$. For large enough $\lambda$, the most efficient search strategy is a Lévy walk with stability parameter close to $\alpha=1$. When the search is destructive, then the most efficient strategy is to move in a ballistic fashion, i.e., with the stability exponent $\alpha=1$.
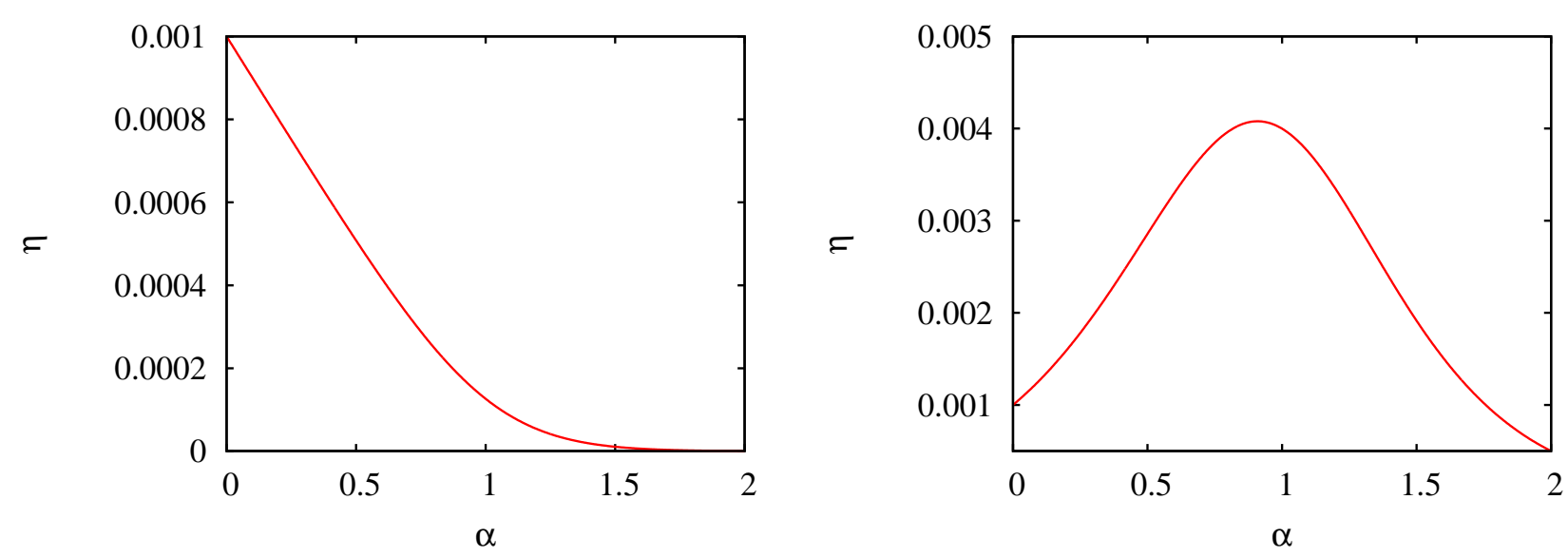

Figure 2.1: The efficiency function $\boldsymbol{\eta}(\boldsymbol{\alpha})$ for a destructive search (left panel) and nondestructive search (right panel) with $r_{v}=1$ and $\lambda=1000$.

In the example above, the quantity to be minimized by changing the parameter $\alpha$ is the average distance covered between two consecutive targets. If we assume that the searcher moves with constant velocity throughout the search process, then minimizing the time to find the necessary targets becomes equivalent to minimizing the total distance covered. Generally, random search processes rely on the calculation of the mean first passage time to a target. This is the probability $f\left(\boldsymbol{x}, t ; \boldsymbol{x}_{\mathbf{0}}\right)$ that a target located at $\boldsymbol{x}$ is found for the first time after time $t$, given that the search process started at $\boldsymbol{x}_{\mathbf{0}}$. In most cases it is difficult to obtain the entire distribution of the first passage times [27], so most of the results reported in the literature are based on the mean values. Although such models based on mean values give ample insight into the optimisation problem, much detail is lost and the complete picture cannot be seen.

Consider a foraging animal that during its daily search for food has to find a minimum amount in order to survive. Let us say that this amount is $N$ units or targets. Besides the food requirement, another restriction that is involved is the amount of resources available to 
the forager. The amount of resources will determine how much time $T$ can be dedicated to the search. It could be in the form of energy available to move continuously for a certain time or it could be the amount of daylight time available for a productive search. What is the probability of finding all the $N$ units or targets in the available time $T$ and which strategy maximises this probability? Another example would be a rescue mission in which a helicopter searches for survivors. The task is to find all of the $N$ survivors in a limited amount of time $T$ with probability 1, i.e., with certainty. In such cases, the time restrictions would be related to the amount of resources available for the search and the time the survivors can remain under the adverse conditions without assistance.

\subsubsection{Random Search on a Lattice}

A random search on a lattice, which can also be considered as a discrete search, implies that there are restrictions in the type of movements that can be performed by the searcher. In the simplest case of a square lattice, the one that we shall use, the searcher can only jump to its four nearest neighbours so that only $90^{\circ}$ turns are possible. A levy walk is approximated on the square lattice by assuming that between two consecutive turns the searcher makes a series of $n$ nearest-neighbour jumps in the same direction, where $n$ is an integer sampled from a Lévy stable distribution. The targets are discovered when the searcher lands on the lattice point containing them; there is no need to define a detection or visibility radius as in the continuous case.

The lattice model might seem oversimplified and unrealistic, yet there exists at least one case in the animal kingdom where it does apply. It is know that the common fruit fly (Drosophila melanogaster) explores its environment using straight flight paths that are interrupted by rapid $90^{\circ}$ turns, or body-saccades [28]. A detailed study of the foraging habits of the fruit fly was recently done by Reynolds and Frye [29]. They performed experiments and recorded the trajectories of the flies while they explored the space allocated to them. By analysing the trajectories, they concluded that the fruit flies adopt a Lévy search strategy with stability parameter $\alpha \approx 2.1$. They also use numerical simulations to show that the optimum search strategy corresponds to turning angles that are equal to or greater than $90^{\circ}$. 


\section{Chapter 3}

\section{Convex Hulls and its Applications in Ecology}

In the previous chapter we have given evidence of the existence of Lévy walks in nature and we have pointed out their relevance. There exist circumstances under which the Lévy walk is the most efficient uncorrelated random search strategy. At this point we can ask ourselves how we can identify a Lévy walk strategy from a given data set.

The convex hull of a set of points $X$ in the Euclidean plane is defined as the smallest convex polygon that contains all the points in $X$. Consequently, the set of points that form the convex hull will be a subset of $X$. A convex set is one in which the line segment joining any two points of the set is itself fully contained in the set. If $X$ consists of a countable number of points, then its convex hull will be the minimum convex polygon that encloses all of them. If we randomly hammer some nails onto a wooden board and then place a rubber band so that it surrounds all of the nails, then the rubber band will create the minimum convex polygon around the nails. Alternatively, one can think of a drunken gardener [30] dropping seeds while moving around the garden. The minimum length of fencing required to enclose all the flowers once they have bloomed is the convex hull around the flowers. The concept of a convex hull can be generalised to more than two dimensions in which case one has to look for the minimum convex polytope. In case the points in $X$ are randomly distributed on the plane, then we speak of random convex hulls whose geometric characteristics such as the perimeter, area, number of vertices, etc. are themselves random variables. The random points may either be independently chosen from an identical distribution or they could be correlated, like the turning points of a planar random walk of $N$ steps.

It is often desirable to quantify the geometry of the space covered by the sample path of a random process. In ecology, for example, one is interested in the estimation of the home range of an animal or a group of animals, defined as the space that they use for their regular activities. Accurate home range estimates are important in habitat conservation planning [31, 32, 33] and for understanding a species' spatial and behavioural tendencies [34]. For this, one requires information about the geometry of the home range and how it evolves in time. Since the 
motion of many foraging animals is approximately random, one is naturally interested in the geometric properties of two-dimensional stochastic processes $[6,35,5]$. A simple and widely employed approach to quantify the area covered by a random two-dimensional motion involves the use of convex hulls. This method presents some limitations, mainly that of not being able to distinguish between areas with high and low visit density. However, there are many cases where it is the preferred and more reliable method [14]. An alternative approach is using kernel estimators. However, according to Boyle et al. not everyone in the field agrees that they represent the best method for determining home range sizes [14].

The convex hull of a random process is strictly related to the extreme values because it is comprised of the points that lie on the boarder created by the process. Hence, when we study the evolution of the convex hull, we actually study how the boarders spread out over a surface. Such dynamics is very important in the study of the spread of diseases or contaminants, since in these cases it is important to know how the front of the disease or contamination spreads and not the mean square displacement for example. In the context of branching Brownian motion, convex hulls have been proposed as a way to characterize the spatial extent of epidemics in animals at the early outbreak stage [36].

While the calculation of properties of a convex hull of uncorrelated random points is rather an old problem, much effort has recently been put into the investigation of the convex hull of one or more Brownian motions and Lévy flights [37]. For Brownian motion it is possible to analytically evaluate the average perimeter length and the average area of the random convex hull (for a review see [13]). An important tool that facilitates the process of working out the average properties of random convex hulls in general is a formula due to Cauchy. We will discuss the formula in more detail in chapter 4 . Nevertheless, it seems that even with this formula, determining the distributions of quantities such as the perimeter or area remains a challenge. In the case of simple planar random walks, for example, the analytical expressions for the distribution of the perimeter and area have still not been determined. Moreover, seemingly simple problems such as that of calculating the distribution of the diameter of the smallest circle entirely containing a random walk, appears still to be unresolved [38].

In this chapter, one of our goals is to develop new insights regarding the distributions of the properties of convex hulls. Where possible, we use an analytical approach. Otherwise, we consider the properties of convex hulls from computer generated trajectories of planar random walks (normal diffusion) and scale-free Lévy walks (super-diffusion).

In order to numerically determine the convex hull of a single trajectory we use a simple and well known algorithm known as the Graham scan [39]. The Graham scan is restricted to two dimensions, but there exist algorithms such as QuickHull [40] that work also in higher dimensions.

As was discussed in the previous chapter, there is growing evidence that there exist cases in which certain animal species adopt Lévy walks as a search strategy in order to maximise their chances of finding enough food for their survival, especially in regions where food sources are 
scarce and sparsely distributed [20]. We propose an algorithm that makes use of convex hulls of random processes as a way to determine whether the random search pattern of a forager can be modelled with a Lévy type walk or a normal random walk.

\subsection{Convex Hulls of Planar Brownian Paths}

The exact expression for the time evolution of the mean perimeter and area of the convex hull of $N$ independent planar Brownian paths was presented recently by Majumdar et al. [37]. Using the standard scaling property of Brownian motion, whereby the length scale increases as the square root of the time scale, it turns out that the mean perimeter and area of the global convex hull of $N$ independent Brownian paths will, respectively, behave as $\left\langle L_{N}\right\rangle=\alpha_{N} \sqrt{T}$ and $\left\langle A_{N}\right\rangle=\beta_{N} T$ for all $T$. Recently, the authors of [37] derived the exact expressions for $\alpha_{N}$ and $\beta_{N}$, while the exact expression for the average perimeter of a single Brownian path was calculated earlier, in 1980, by Takács [41] and the average area, in 1983, by El Bachir [42].

For the sake of clarity in the discussion that is to follow, we will use discrete random walks as our model for normal diffusion instead of Brownian motion. The random walk tends to Brownian motion as the diffusive limit is approached. We shall also use the term Brownian convex hull for a convex hull that encloses a planar random walk.

As far as the probability distribution of the perimeter of the Brownian convex hull is concerned, the most recent developments are presented in the paper by Wade and Xu [30]. The authors studied how the distribution of the perimeter of the Brownian convex hull evolves as a function of the number of steps taken by the underlying random walker. It is important to note, however, that their results are valid only for random walks with a drift, where the jump lengths are sampled from a bivariate Gaussian distribution that is not centred at zero. They proved a central limit theorem for the convex hull perimeter of a biased random walk: in the presence of drift, the distribution of the perimeter of the Brownian convex hull converges to a Gaussian as the number of steps tends to infinity. However, the paper does not offer any insight on why the theorem is not valid for random walks without drift. In this section we present a compelling argument, in terms of the trapping times inside the instantaneous convex hull, that explains why the perimeter distribution of a Brownian convex hull does not tend to a Gaussian.

\section{Perimeter Distribution of the Brownian Convex Hull}

Consider a sequence of independent, identically distributed (i.i.d) random variables $X_{1}, X_{2}, \ldots$ in $\mathbb{R}^{2}$ with finite mean $\boldsymbol{\mu}=\left\langle X_{i}\right\rangle=\left(\mu_{x}, \mu_{y}\right)$ and variance $\sigma^{2}=\left\langle\left\|X_{i}\right\|^{2}\right\rangle-\|\boldsymbol{\mu}\|^{2}$. The sum of such random variables corresponds to a planar random walk $S_{n}=\sum_{i=1}^{n} X_{i}$, where $S_{n}$ is the position of the random walker in $\mathbb{R}^{2}$ after $n$ steps. The presence or absence of drift in the random walk depends on whether $\boldsymbol{\mu} \neq 0$ or $\boldsymbol{\mu}=0$ respectively. We will use $L_{n}$ to denote the length of the perimeter of the convex hull enclosing the random walk after $n$ steps. Notice that $L_{n}$ is itself a random variable in $\mathbb{R}$ and is such that $\Delta L=L_{n+1}-L_{n} \geq 0 \forall n \in \mathbb{N}$. In other 
words $L_{n}$ is a non-negative and non-decreasing function of $n$. The exact expression for the expectation of $L_{n}$ is known and it is given by [43]

$$
\left\langle L_{n}\right\rangle=2 \sum_{k=1}^{n} \frac{1}{k}\left\langle\left\|S_{k}\right\|\right\rangle,
$$

from which it follows that in the limit of large $n,\left\langle L_{n}\right\rangle \sim n$ when $\boldsymbol{\mu} \neq 0$ and $\left\langle L_{n}\right\rangle \sim \sqrt{n}$ when $\boldsymbol{\mu}=0$. The expression for the variance of $L_{n}$ can be determined only in the limiting case $(n \rightarrow \infty)$ with $\boldsymbol{\mu} \neq 0$ [30]. Figure 3.1 shows the distribution of $L_{n}$ in the absence of drift (left panel) and in the presence of drift (right panel). The results of the simulation resemble the ones presented in figure 3 of [30], confirming that the distribution converges to a Gaussian in the presence of drift and that it is uncertain what the limiting form is in the absence of drift.
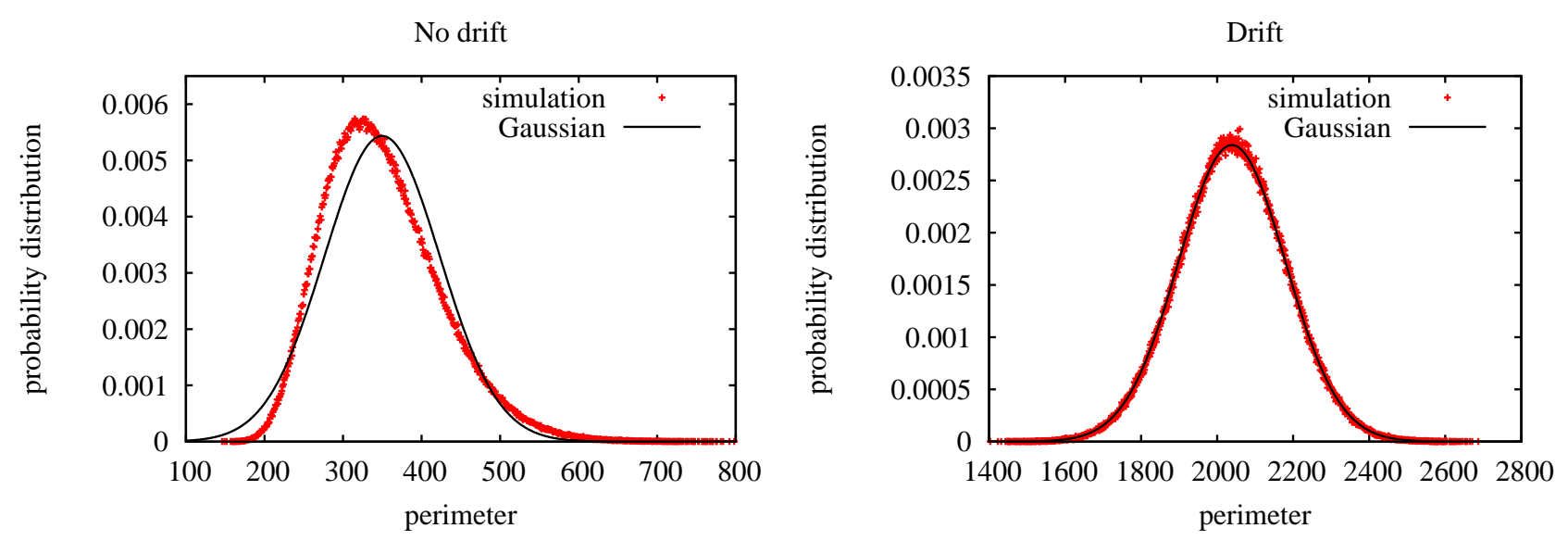

Figure 3.1: Perimeter of a convex hull of a planar random walk in the absence of drift (left) and in the presence of drift (right). In both panels the black line is the Gaussian distribution with mean and variance obtained from the simulations. The underlying random walk consists of a total of $n=5000$ steps with $\sigma^{2}=2$ and $\boldsymbol{\mu}=(0,0)$ (left panel) and $\boldsymbol{\mu}=(0.2,0)$ (right panel). After $n=5000$ steps of the planar random walk, the corresponding perimeter distribution remains asymmetric and non-Gaussian when there is no drift. In the presence of drift, a theorem by Wade and Xu [30] states that the perimeter distribution converges to a Gaussian distribution for $n \rightarrow \infty$. This is confirmed by the right panel.

Let us assume that after making the $n^{\text {th }}$ jump, the random walker increases the perimeter $L_{n}$ by some random amount $\Delta L$. Let us further assume that the $(n+1)^{\text {th }}$ jump takes the walker away from the hull, towards the centre so that the perimeter length remains unchanged, i.e. $\Delta L=0$. We can ask ourselves the following question: After how many jumps $\tau$ will the walker escape from the convex hull $\mathcal{C}_{n}$ created after the $n^{\text {th }}$ jump? For how long will $L_{n}$ remain constant after the $n^{\text {th }}$ step? We will use the properties of these trapping times, or waiting times $\tau$, to present an intuitive explanation for the results shown in figure 3.1. 

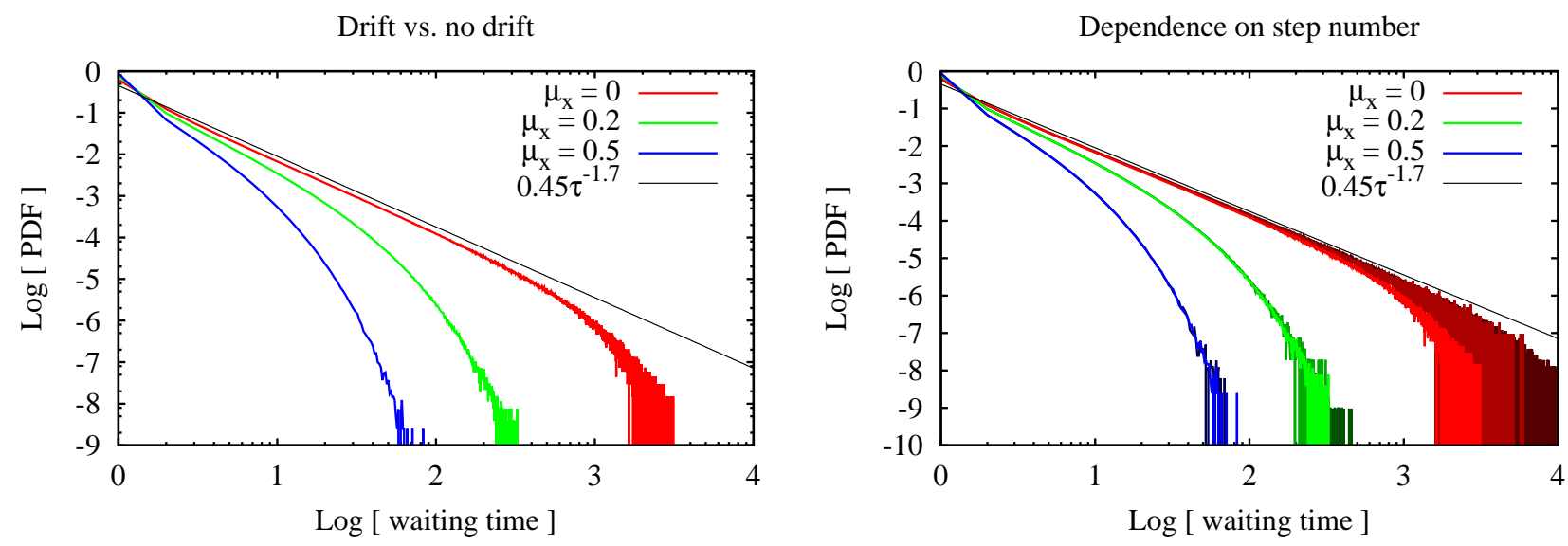

Figure 3.2: Distribution of trapping or waiting times $\tau$ between consecutive increments of the convex hull of a planar random walk. The underlying random walk is composed of jumps sampled from a Gaussian distribution with $\sigma^{2}=2$. Left panel: The distribution depends on the drift strength $\boldsymbol{\mu}=\left(\mu_{x}, 0\right)$. In the absence of drift (red curve), the distribution resembles a truncated inverse power law. The straight black line serves as a guide. In the presence of drift (green and blue curves) the distributions decay rapidly without showing signs of a power law with exponent $\gamma=-1.7$. In all three cases, the number of steps is the same, $n=5000$. Right panel: Dependence of the distribution on the total number of steps $n$. The different colours represent different values of $\boldsymbol{\mu}$ and the different shades of the same colour represent different values of $n: 5000,10000$ and 20000. In the presence of drift (green and blue curves), the distributions are the same for the three different values of $n$ and the cut-off is around $\tau=60$ for $\mu_{x}=0.5$ and $\tau=400$ for $\mu_{x}=0.2$. In the absence of drift (red curves) the domain of the power law increases with $n$.

We start by considering the distribution of the waiting times. Figure 3.2 shows some examples based on numerical simulations of waiting time distributions for the case where the jumps are sampled from a Gaussian distribution, i.e. $X_{i} \sim \mathcal{N}\left(\boldsymbol{\mu}, \sigma^{2}\right)$. When $\boldsymbol{\mu}=0$ the results presented in the figure indicate that the waiting times $\tau$ are distributed according to a truncated power law with the exponent $\gamma=-1.7$. As the number of steps is increased (right panel of figure 3.2) the cut-off point shifts to larger values of $\tau$, thereby extending the domain of the power law. This suggests that the cut-off is introduced by the total number of steps $n$ that take place, which we will also refer to as the observation period. Since $\tau$ is broadly distributed, the trapping times can be of the order of the observation time. On the other hand, when $\boldsymbol{\mu} \neq 0$ a genuine (intrinsic) cut-off is introduced in the waiting time distribution that is independent of the observation time. This creates a well-defined characteristic waiting time of the order of $\langle\tau\rangle$. In support of this argument is the numerical result shown in the right panel of figure 3.2. The green and blue coloured curves correspond to cases where $\boldsymbol{\mu} \neq 0$ (drift present) and the 
different shades correspond to different values of $n$. This suggests that in the case where drift is present, the waiting time distribution is independent of $n$, which is not the case for $\boldsymbol{\mu}=0$ (curves with shades of red).

Let us now go back to the derived stochastic process $\left\{L_{n}\right\}$. We have already established that it is always positive with positive increments. However, the random variables $L_{1}, L_{2}, \ldots$ are not independent, regardless of the value assigned to $\boldsymbol{\mu}$. The number of steps $i$ that have to take place in order to lose the correlation between two random variables $L_{n}$ and $L_{n+i}$ is of the order of the mean waiting time $\langle\tau\rangle$. Therefore, if we observe the process for a very large number of steps, $n \gg\langle\tau\rangle$, then we can assume statistical independence and that $p\left(L_{n}\right)$ is a Gaussian distribution. The problem arises when $\boldsymbol{\mu}=0$ and therefore $\langle\tau\rangle$ is necessarily of the order of the observation time $n$. In that case the transient period is never overcome, it persists throughout the observation period. For this reason $p\left(L_{n}\right)$ assumes a shape that is not Gaussian for all values of $n$. The fact is that the greater the value of $n$ the bigger the convex hull and we expect that the chances of the random walker getting trapped for a very long time increases.

In the particular case where $\boldsymbol{\mu}=(0.2,0)$ and $\sigma^{2}=2$ the simulations show that the average value of the waiting time is $\langle\tau\rangle=7.01$. Figure 3.3 shows an example of $p\left(L_{n}\right)$ during the transient phase $(n \approx\langle\tau\rangle)$. From the figure, we can also see that as soon as $n$ is greater than the maximum value of $\tau$, then $p\left(L_{n}\right)$ starts taking the form of a Gaussian distribution.

We have explained under what conditions and why there is convergence of $p\left(L_{n}\right)$ to a Gaussian distribution, but we have not discussed why the waiting time distribution has a cutoff that is independent of $n$ when $\boldsymbol{\mu} \neq 0$. For the sake of argument let us consider a random walk with a bias towards the positive $x$ direction on a plane, i.e. at every jump the random walker is more likely to move right rather than in any other direction. Consequently, most of the escapes will occur over the right-most edge of the convex hull. When $n \gg\langle\tau\rangle$, the probability that the random walker wanders off to the centre of the hull and then exits through the left-most edge will tend to zero. Note that because of the bias, the hull will be elongated along the $x$-axis for any $n$ (See the right panel of figure 3.4). Thus, for large $n$ the random walker will reach a stationary state in which the exit time distribution does not change because it experiences only the right-most edges of the convex hull, unaffected by the borders on the opposite side.

Every time the convex hull increases in size, the random walker will be found on one of the vertices of the newly created hull before it continues moving further. This situation resembles significantly the problem of random walks inside a wedge domain with absorbing boundaries and an opening angle $\theta$ (figure 3.5). Since the hull is convex, the angle of the wedge can only be in the range $(0, \pi)$. The trapping times $\tau$ within the wedge are distributed according to [44]

$$
\psi(\tau)=\frac{\pi}{2 \theta}\left(\frac{r_{0}}{\sqrt{D}}\right)^{\pi / \theta} \tau^{-1-\frac{\pi}{2 \theta}}
$$

where $r_{0}$ is the starting position of the random walk and $D$ is the diffusion constant. For our 


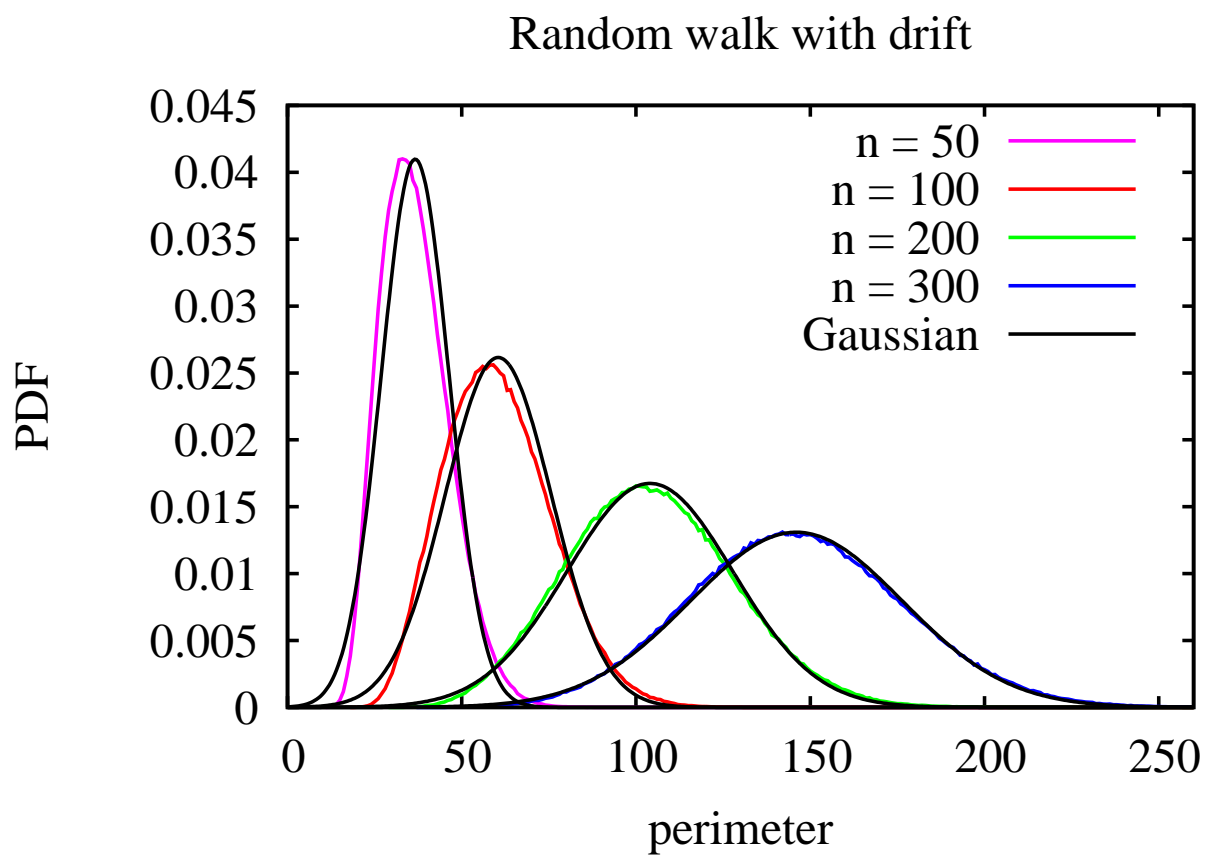

Figure 3.3: Convergence of the convex hull perimeter distribution to a Gaussian

- Random walk with drift. Each coloured curve corresponds to a different step number n. The jump lengths are sampled from a Gaussian distribution with mean $\boldsymbol{\mu}=(0.2,0)$ and variance $\sigma^{2}=2$. The black curves are Gaussian distributions with the mean and variance obtained from the numerical data. As $n$ increases the distribution changes shape from a skewed distribution to a symmetric Gaussian distribution. The time of convergence is of the order of the cut-off time of the distribution corresponding to $\mu_{x}=0.2$ shown in figure 3.2 (green curve). 
purposes we will only consider random walks that start from the apex of the wedge which can be approximated by the condition $r_{0}=1$ for a discrete random walk. If $\theta$ is greater than $\pi / 2$ the exponent of the inverse power law in 3.2 will be smaller than 2, causing the mean trapping time to diverge.

In the presence of drift, we make the assumption that the trapping time statistics of the wedge domain is similar to that of the convex hull. This is based on the fact that the drift keeps the random walker close to the vertex of the hull or close to the apex in the case of the wedge. As mentioned earlier, at a certain point the trapping times become independent of the hull size. The only difference is that as the hull grows the random walker will move from an old vertex to a newly created one with a different opening angle. Consequently it is reasonable to assume that in the presence of drift the waiting time statistics of a hull reduces to the wedge problem with randomly changing opening angles $\theta$. In the presence of drift, the waiting time distribution is not an inverse power law, but instead it decays very rapidly. Figure 3.6 shows the results of numerical simulations in which a random walker was confined to move in an infinite wedge domain. Each panel of the figure corresponds to a different opening angle $\theta$. The rapidly decaying curve that corresponds to the case with drift (blue) is very similar to the green curve representing the waiting times related to the convex hull growth shown in figure 3.2. These two cases are shown together in figure 3.7 where the waiting time distributions obtained for a random walker confined to move in a growing hull is compared to a the same random walker in an infinite wedge domain. In the driftless case, the waiting time distribution of the hull decays with an inverse power exponent of $\gamma=1.7$ (see figure 3.2), which according to 3.2 corresponds to a wedge domain with the angle $\theta=0.5 \pi / 0.7$. Since the the the hull vertex angles are random, we can use this result to form a conjecture which states that the average or most dominant apex angle is around $\theta=0.5 \pi / 0.7$.

In the absence of drift, the correspondence holds to a certain degree if we limit the extent of the wedge by adding an arced boundary in front of the apex, forming a sort of a pie wedge. In particular, this creates a cut-off in the tail of the power law in (3.2). When there is an absence of drift $(\boldsymbol{\mu}=0)$ or during the transient period ( $n$ of the order of $\langle\tau\rangle$ ) when the size of the hull is small, the random walker has the chance to explore the entire convex hull with a good probability of escaping through one of the opposite edges from where it started. In these cases the waiting time distribution is a truncated inverse power law with an exponent of 1.7, which means that for time scales below the cut-off limit it is practically scale-free. Consequently, it should be expected that very large waiting times, compared to the average waiting time present due to the cut-off, become frequent. This argument can help explain the presence of asymmetry in the perimeter length distribution $p(L, T)$, which is biased towards smaller values of $\tau$ with respect to the Gaussian distribution with the same mean and variance (see figure 3.1). In the case of drift, the skewness is lost when the waiting time starts decaying exponentially while in the absence of drift, the heavy tail persists and consequently so does the skewness of the propagator. In contrast, the propagator fails to converge to a Gaussian distribution when the 
drift vanishes.
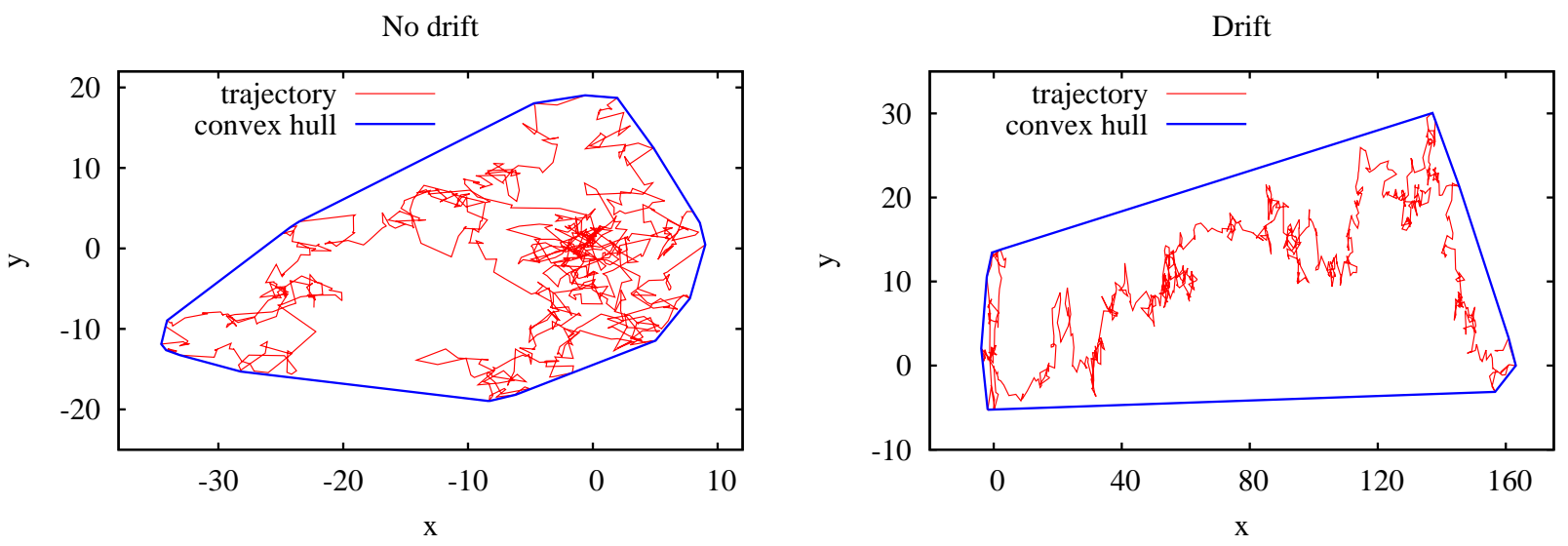

Figure 3.4: Convex hull of a planar random walk. A typical example is shown for the case in the absence of drift (left panel) and in the presence of drift (right panel). As a result of the drift, the convex hull becomes visibly elongated along the x-axis.

\section{Distribution of the Span of a One-dimensional Random Walk}

In order to obtain a clearer picture, we will now study the one-dimensional equivalent of what was considered above. We consider the simplest possible case; the convex hull of a one-dimensional random walk. One might argue whether it makes sense to talk about convex hulls in one dimension, so to avoid any ambiguity we will consider its span or extent, which is closely related to the maximum excursion. We will examine how the span of a random walk evolves with time, just as we did for the planar random walk and its convex hull. The evolution of the span can be related to the well established problem of first passage times of a random walker constrained to move on a finite interval. We shall use the results obtained in the onedimensional case to justify the intuitive explanations offered for the two-dimensional process earlier in this chapter.

If $x(t)$ is the trajectory of the random walk, then the span of the process at time $T$ is defined to be the difference between $\max _{t \in[0, T]}\{x(t)\}$ and $\min _{t \in[0, T]}\{x(t)\}$. The study of the span of a one-dimensional random walk was initiated by H.E. Daniels [45], who provided the exact expression for its probability distribution. Subsequently, the problem of the span became very popular in the context of polymer configurations [46]. More recently, Weiss and Rubin [38] expanded the idea to continuous time random walks and Lévy flights. In the case of two or more dimensions, the spans are defined as the dimensions of the smallest box with sides parallel to the coordinate axes that entirely contain the random walk.

Before proceeding, we would like to stress again that even in the one dimensional case the stochastic process $\left\{L_{n}\right\}$ departs from a simple Gaussian process in two significant ways. 

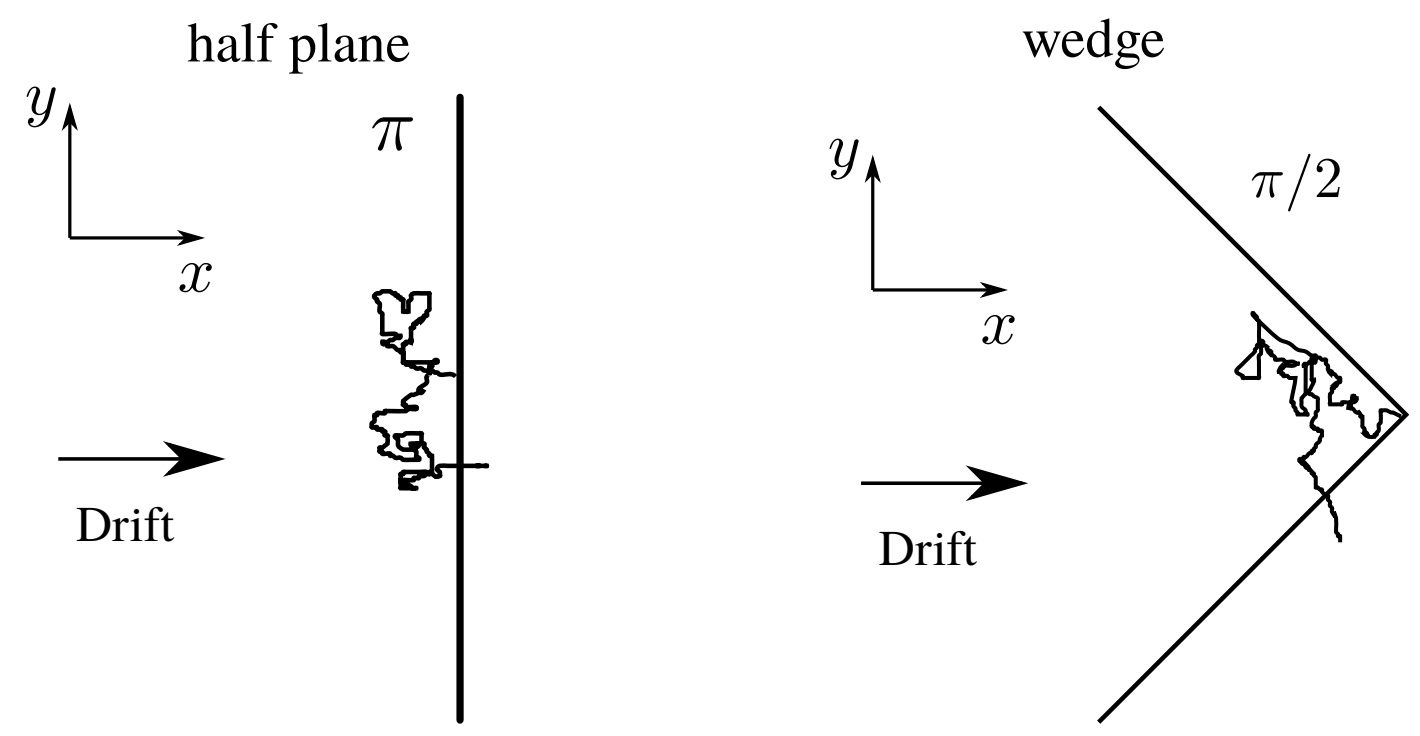

Figure 3.5: Random walk in a wedge domain. Examples of wedge domains for two different values of $\theta$ are shown. In the presence of drift, the neighbourhood of the vertex of a convex hull can be approximated by an infinite wedge domain. The drift keeps the random walker in the "vicinity" of the wedge apex before it hits one of the edges. Similarly, a random walker inside its convex hull moves in the "vicinity" of one of the vertices because of the drift.

Plane $-\vartheta=\pi$

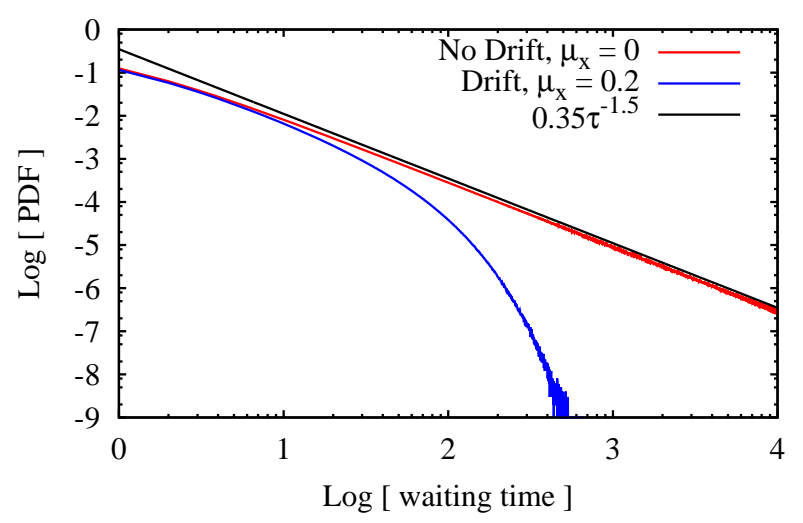

Wedge $-\vartheta=\pi / 2$

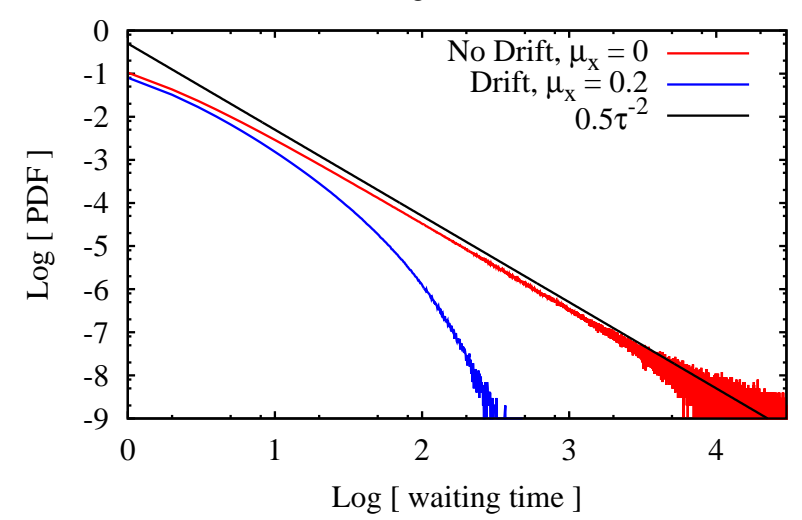

Figure 3.6: Waiting time distributions for different wedge domains. The coloured curves are results of numerical simulations. The two panels correspond to wedge domains with different opening angles. The underlying process is a random walk with $\sigma^{2}=2$ and $\boldsymbol{\mu}=0$ (red curve - no drift) or $\boldsymbol{\mu}=(0.2,0)$ (blue curve - drift). The black curves are the theoretical results for random walks without drift starting from the apex of an infinite wedge domain (see (3.2)). 

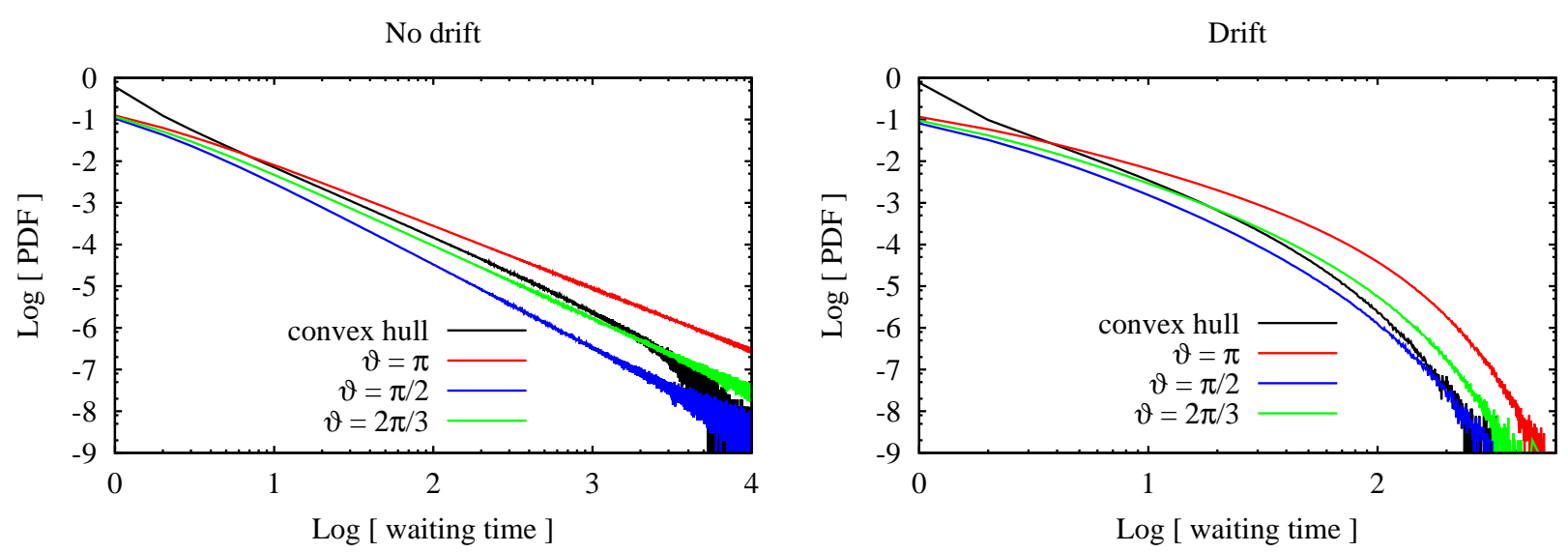

Figure 3.7: Waiting time distributions for the expanding convex hull (black curve) and various wedge domains (coloured curves). For every curve, the underlying process is a random walk with $\sigma^{2}=2$. In the case of the expanding convex hull the underlying random walk is comprised of $n=20,000$ steps.

First, the waiting times are governed by an inverse power law and secondly, their average value increases with the elapsed time of the process.

\section{Random walk in the presence of drift}

Consider a one-dimensional random walker with a drift that is confined to move within the interval $[0, L]$ with absorbing boundaries. If the jump lengths are sampled from a normal distribution with mean $\mu>0$ and variance $\sigma^{2}$, then, in the diffusive limit, the equation governing the evolution of propagator $p(x, t)$ of the random walk is the convection-diffusion equation of the form

$$
\frac{\partial p(x, t)}{\partial t}+v \frac{\partial p(x, t)}{\partial x}=D \frac{\partial^{2} p(x, t)}{\partial x^{2}}
$$

where $v=\mu / \tau$ and $D=\sigma^{2} / \tau$ and where $\tau=1$ is the characteristic time taken for each step. It can be shown (see for example reference [44]) that the probability that the random walker remains inside the interval upto a time $t$ is, in the asymptotic limit, given by an exponentially decaying function with characteristic time $\tau_{c}$, where

$$
\tau_{c}=\frac{L^{2}}{\pi^{2} D} \cdot \frac{1}{1+\left(\frac{v L}{2 \pi D}\right)^{2}} .
$$

We assume that the process stops when one of the boundaries is reached. From (3.4) we can deduce that the characteristic survival time is independent of $L$ whenever $\left(\frac{v L}{2 \pi D}\right)^{2} \gg 1$ or, more simply, when $v \gg D / L$. Therefore, if we keep $v$ constant, then for large enough $L$ we will have $\tau_{c}=D / v^{2}$, which is characteristic of the strongly biased regime. Furthermore, if we assume 
that $v$ is positive, then the random walker will exit at $x=0$ only if it is initially within a skin depth $D / v^{2}$ of it. If it is further away from $x=0$, then there is enough time for convection to prevail over diffusion, which is the only mechanism to bring the walker towards the left extreme.

The one-dimensional equivalent of a growing convex hull is the time dependent span $\mathcal{S}(T)=$ $x_{\max }(T)-x_{\min }(T)$, where $x_{\min }$ is the minimum and $x_{\max }$ the maximum reached by the random walker in the time interval $[0, T]$. If we assume that the random walker starts at $x=0$, then at $T=0$ we will have that $x_{\max }=x_{\min }=0$ and therefore $\mathcal{S}=0$. At every time step, the interval $\left[x_{\min }, x_{\max }\right]$ can either remain constant or increase. At the early stage of evolution, during the transient period, $\mathcal{S}$ will be smaller than the skin depth, allowing the random walker to escape from both ends of the interval. Consequently, the characteristic trapping time will depend on the size of the interval in the form $\tau_{c}=\mathcal{S}^{2} / D$ so that some memory of the span length will be present in the process. As the span increases, at some point $v \gg D / \mathcal{S}(T)$, and therefore $\tau_{c}$ will become constant and independent of $\mathcal{S}$. All exits will take place to the right so that only $x_{\max }$ increases. This is when the memory is lost and the process becomes Markovian with the right conditions for the probability distribution of the span to converge to the Gaussian distribution.

\section{Random walk in the absence of drift}

It is a well known property of unrestricted one-dimensional random walks that in the absence of drift, the distribution of first passage times follows an inverse power law with exponent $\gamma=1.5$ [44]. In other words, a random walker that starts from an arbitrary point, say $x=0$, will return to that very point for the first time after a random number of steps $\tau$, where $p(\tau) \sim \tau^{-1.5}$. Since the exponent is less than 2 , the average value of $\tau$ diverges so that there is no meaningful characteristic return time. If we place an absorbing boundary at $x=L$ and use $x=0$ as the starting point of the process then the inverse power waiting time distribution acquires an exponential cut-off and hence a characteristic waiting time that depends on $L$ (figure 3.10). Actually, in the case where the random walker starts close to the boarder, the average exit time is of the order of $L$ and of the order of $L^{2} / D$ when away from the boundaries [44]. Therefore, using the second argument, we can estimate that the exponential cut-off will occur at around $\tau=L^{2} / D$.

Finally, as mentioned earlier, the analytical expression for the distribution of the span of a one-dimensional random walk is known $[45,38]$. In the absence of drift, the limiting distribution of the span for large $n$ has the form

$$
p_{\text {span }}(r, n) \approx \frac{8}{\sqrt{2 \pi n \sigma^{2}}} \sum_{j=1}^{\infty}(-1)^{j+1} j^{2} \exp \left(-\frac{j^{2}(r+1)^{2}}{2 n \sigma^{2}}\right)
$$

with mean

$$
\langle r\rangle=\sqrt{\frac{8 n \sigma^{2}}{\pi}} .
$$


In the presence of a bias $\mu$ (drift), we have

$$
p_{\text {span }}(r, n) \approx \frac{8}{\sqrt{2 \pi n \sigma^{2}}} \sum_{j=1}^{\infty}(-1)^{j+1} j^{2} \exp \left(-\frac{(j(r+1)-\mu n)^{2}}{2 n \sigma^{2}}\right)
$$

with mean

$$
\langle r\rangle \sim \mu n
$$

Figures 3.8 and 3.9 show how the equations above compare with numerical results. Equations (3.5), (3.6) and (3.8) are in good agreement with the numerics. However, computer simulations (not shown) suggest deviations from the distribution in (3.7).

In conclusion, the persistent skewness of $p(L, T)$ is a consequence of the inverse power law waiting times. We can draw parallels between a growing two-dimensional convex hull of a planar random walk and a growing one-dimensional span of a simple random walk. The mechanism responsible for keeping the propagator $p(L, T)$ away from the basin of attraction of the Gaussian distribution is the same in both cases.
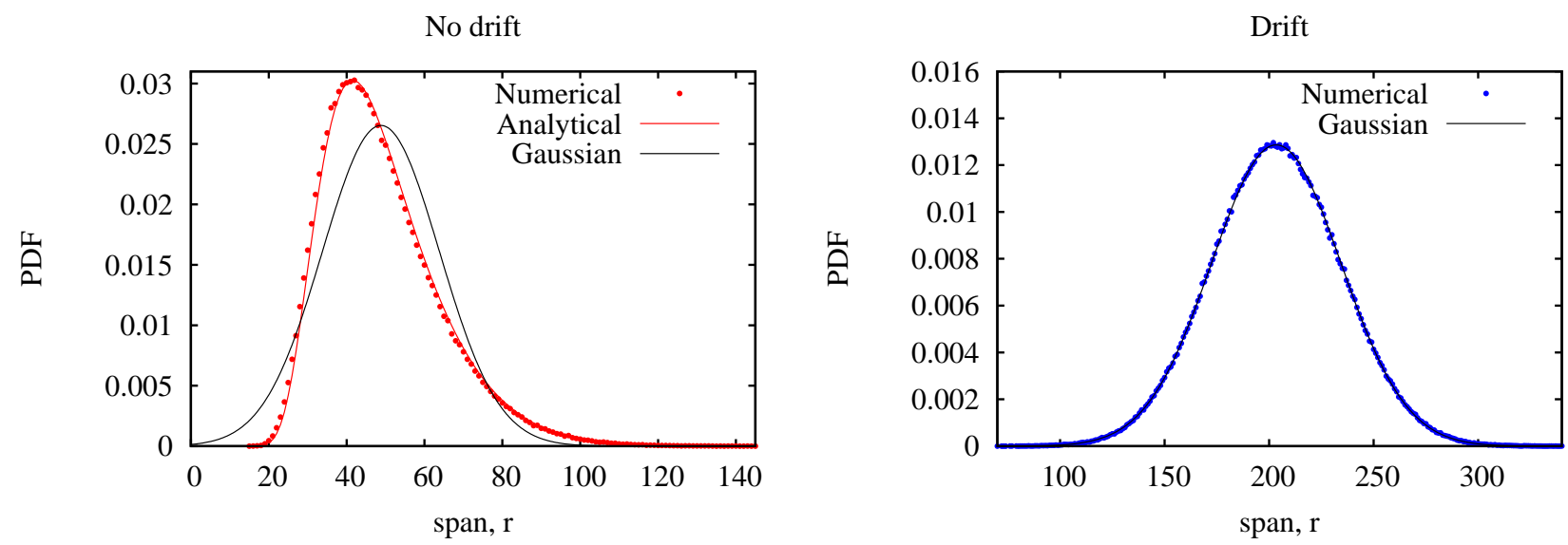

Figure 3.8: Distribution of the span of a random walk. In both panels $n=1000$ steps and $\sigma=1$. Left panel: Shows the case without drift. The red dots were obtained numerically, while the red line is (3.5). The black line is a Gaussian distribution with the mean and variance obtained from the numerical data. Similar to the two-dimensional convex hull case, the span does not tend to a Gaussian distribution. Right panel: Shows the case with drift $(\mu=0.2)$. The blue dots correspond to numerical simulations and fit well the Gaussian distribution (black line). 

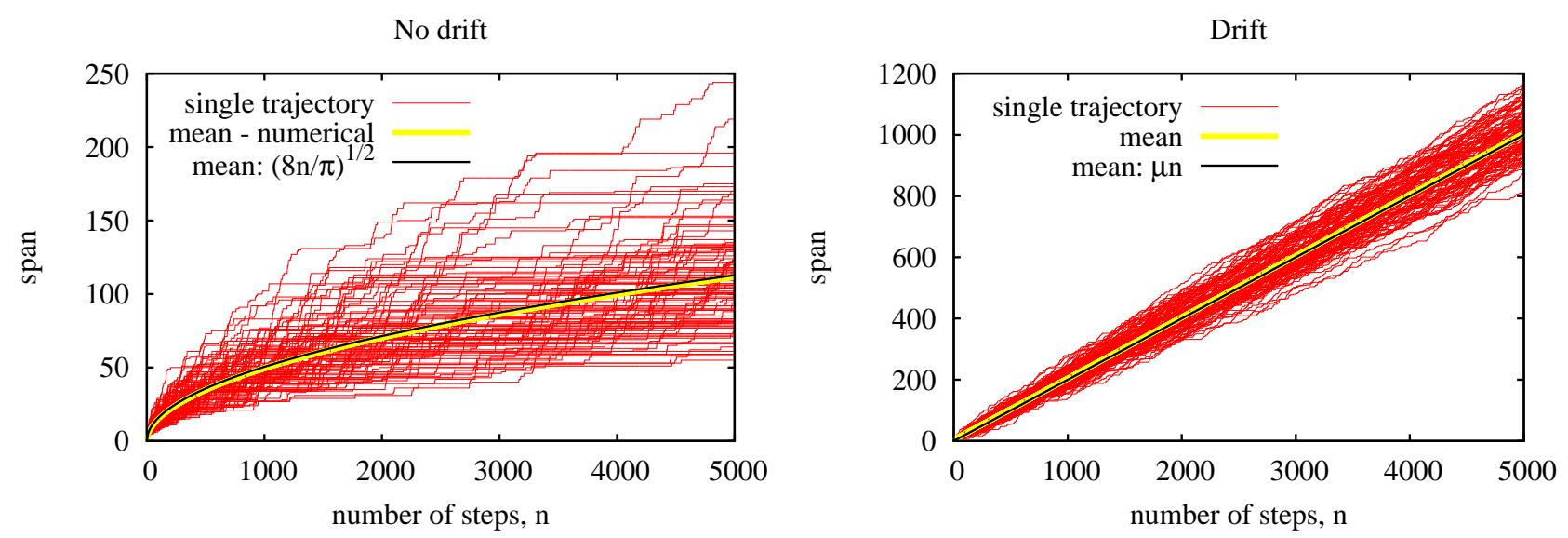

Figure 3.9: The span as a function of the number of steps. In both panels $\sigma=1$. The thin red lines correspond to single trajectories obtained numerically. The thick yellow line is the mean value of the span obtained numerically. The black lines corresponds to the mean values calculated analytically. The left panel is the case without drift and the the right panel is with drift $(\mu=0.2)$.

\section{Approximations of Brownian Convex Hulls}

\section{Perimeter distribution}

In order to better understand the perimeter distribution and to understand the origins of its functional form we try to fit it with some regular functions (note that no exact forms are known). Since the perimeter distribution is related to the problem of first passage times, we use the log-normal (LN) and the inverse Gaussian (IG) distributions for the fit. They have both been found in a variety of first passage time problems. The log-normal model used is

$$
f_{L N}(x)=\frac{1}{x \sqrt{2 \pi} \bar{\sigma}} \exp \left(\frac{-(\ln x-\nu)^{2}}{2 \bar{\sigma}^{2}}\right)
$$

where $\nu \in \mathbb{R}$ and $\bar{\sigma}$ are, respectively, the mean and standard deviation of the variable's natural logarithm; they are the distribution parameters to be determined by fitting. A log-normal distribution with mean $\mu$ and variance $\sigma^{2}$ has parameters $\nu=\ln \left(\mu^{2} / \sqrt{\sigma^{2}+\mu^{2}}\right)$ and $\bar{\sigma}=$ $\sqrt{\ln \left(1+\sigma^{2} / \mu^{2}\right)}$

The inverse Gaussian used is

$$
f_{I G}(x)=\sqrt{\frac{\lambda}{2 \pi x^{3}}} \exp \left(-\frac{\lambda(x-\tilde{\mu})^{2}}{2 \tilde{\mu}^{2} x}\right)
$$

where $\tilde{\mu}>0$ is the mean and $\lambda>0$ the shape parameter. The variance is given by $\tilde{\mu}^{3} / \lambda$.

The results of the fitting are shown in figure 3.11. The mean square difference between the log-normal curve and the data points of the simulation is $1.35004 \times 10^{-8}$. In the case 


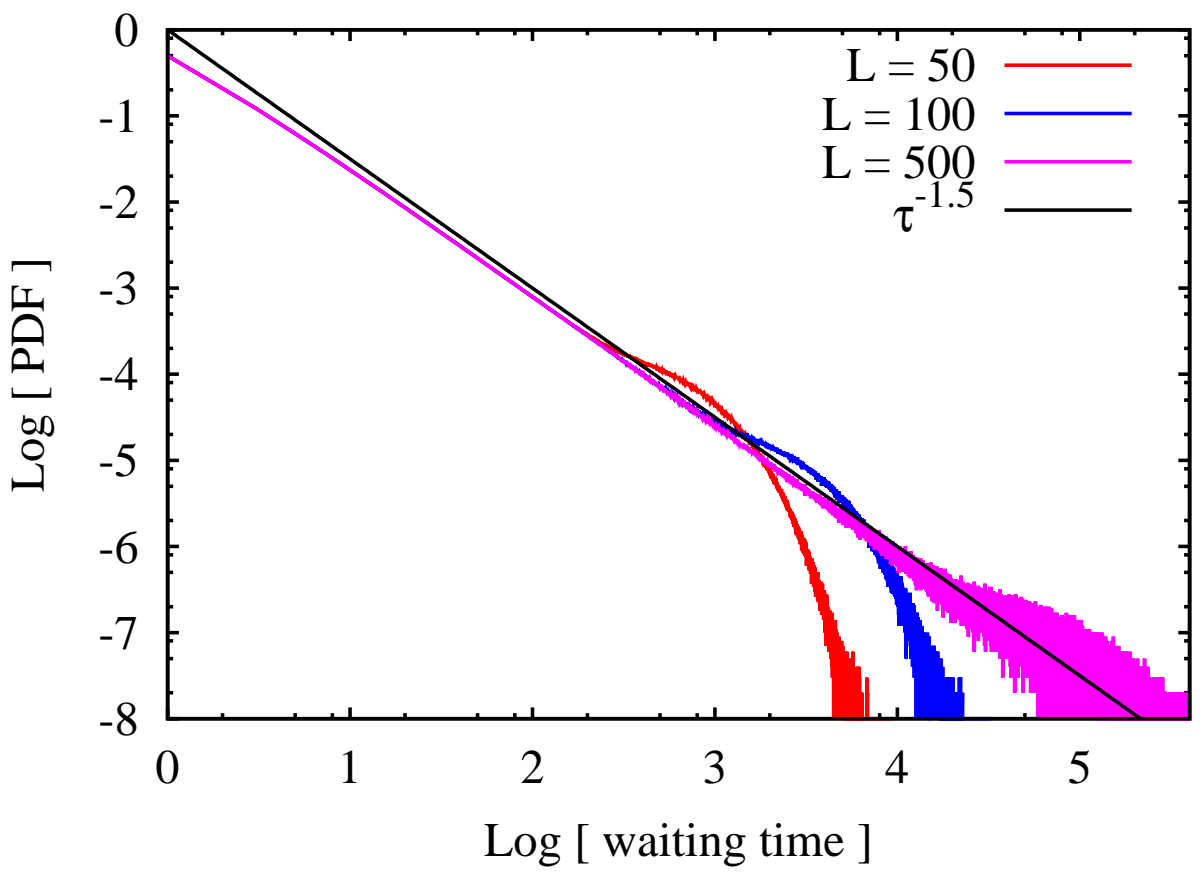

Figure 3.10: Distribution of trapping times inside a finite interval $[0, L]$. The random walk starts at $x=0$ and remain trapped unless $x<0$ or $x>L$. There is no drift and the variance of the single jumps is $\sigma^{2}=1$ so that $D=1$. The bulge that appears for higher values of $\tau$ is due to exits at $x=L$. 
of the inverse Gaussian, the mean square difference from the data points of the simulation is $1.07731 \times 10^{-8}$.

Log-normal case: $\mu=\exp \left(\nu+\bar{\sigma}^{2} / 2\right)=145.002$ and $\sigma^{2}=\left[\exp \left(\bar{\sigma}^{2}\right)-1\right] \exp \left(2 \nu+\bar{\sigma}^{2}\right)=$ 969.719. Inverse Gaussian case: $\mu=\tilde{\mu}=145.025$ and $\sigma^{2}=\tilde{\mu}^{3} / \lambda=961.681$.

A more elaborate analysis of the goodness of fit reveals a substantial deviation in the respective third and fourth standardised moments (see figure 3.12).

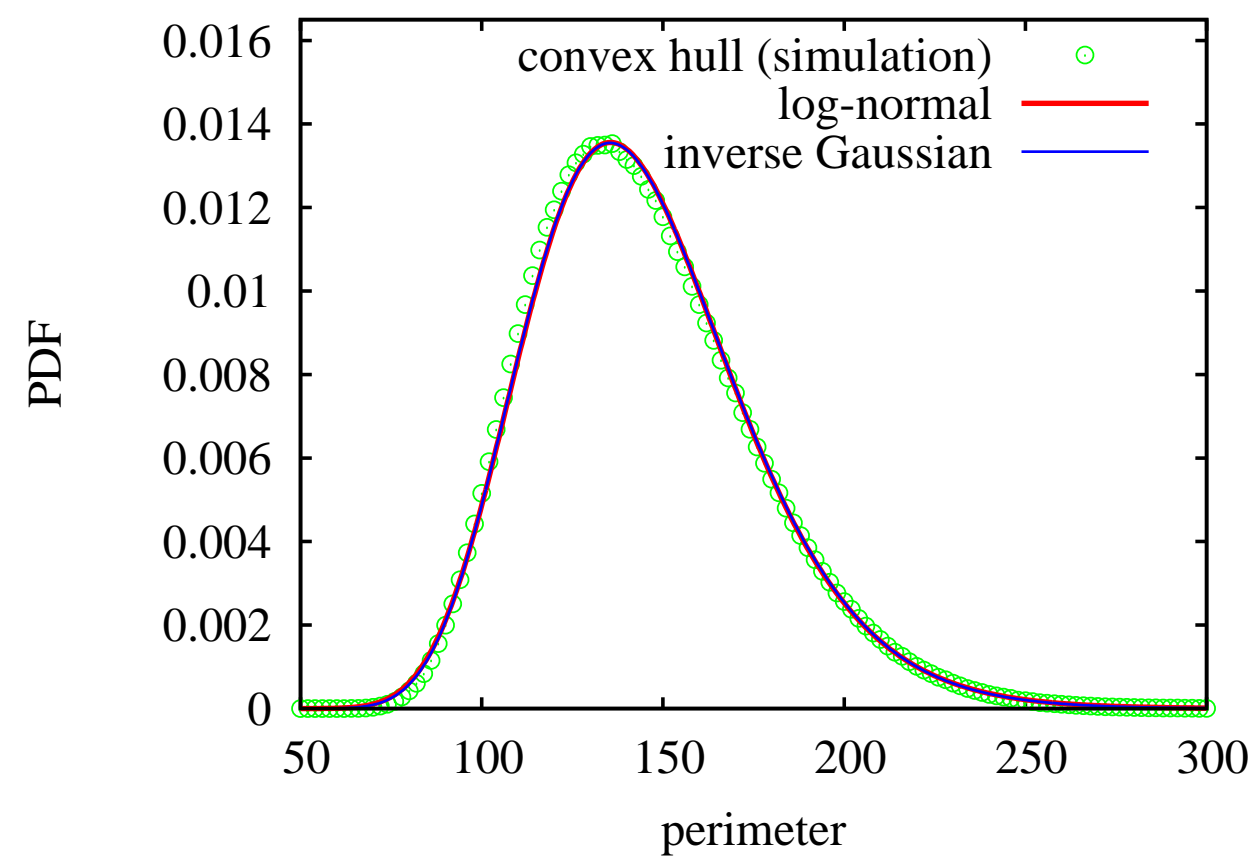

Figure 3.11: Probability density of the convex hull perimeter, L. The underlying planar random walk has 1000 steps and jump sizes of variance two (green circles). The perimeter distribution has mean $\mu=144.581$ and variance $\sigma^{2}=949.317$. The log-normal function in (3.9) fits the data with parameters $\bar{\sigma}=0.212342$ and $\nu=4.9542$ (red curve). The data can also be fitted with an inverse Gaussian with parameters $\tilde{\mu}=145.025$ and $\lambda=3171.76$ (blue curve).

\section{Comparing Brownian convex hulls to regular geometric shapes}

It is well known that the shape of a planar random walk is not spherical [47], regardless of the number of steps taken. There exists a degree of asymmetry that can be quantified using methods that are based on the radius of gyration [48] or the ordered spans of the random walk [49].

As mentioned earlier, the span of a planar random walk is the minimum rectangle that encloses the random walk in such a way that its sides are parallel to the $x$ and $y$ axes (figure 
ME A N

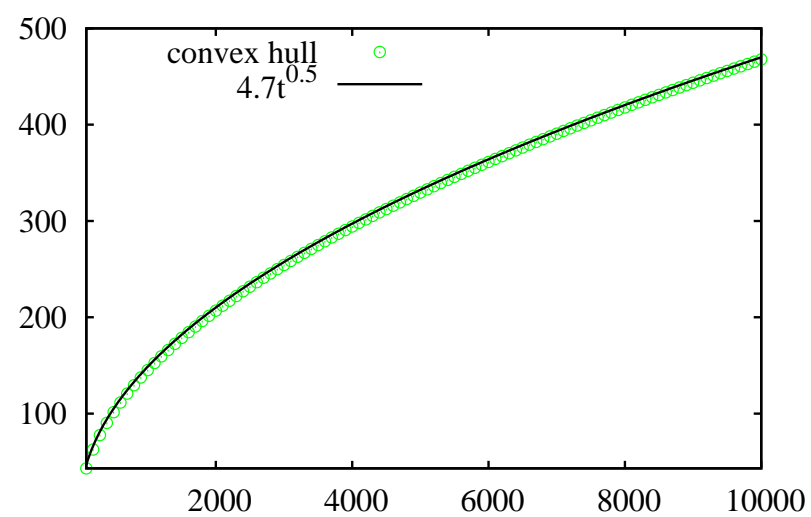

S K E W N E S S

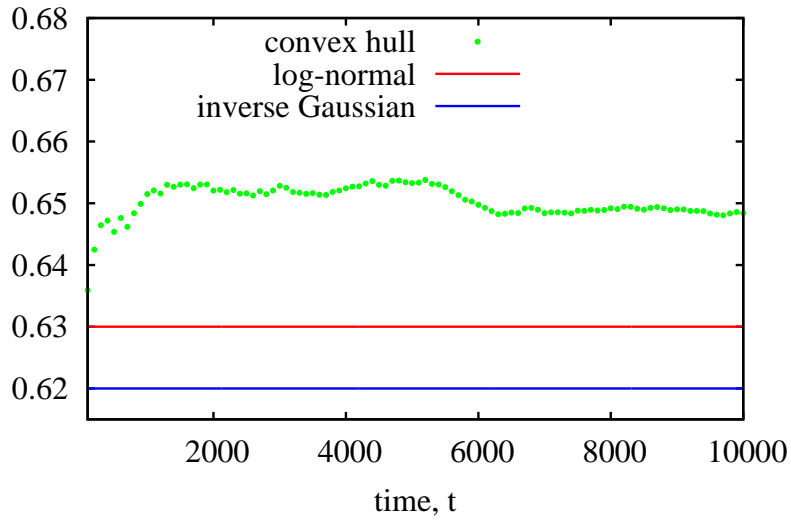

V A R I A N C E

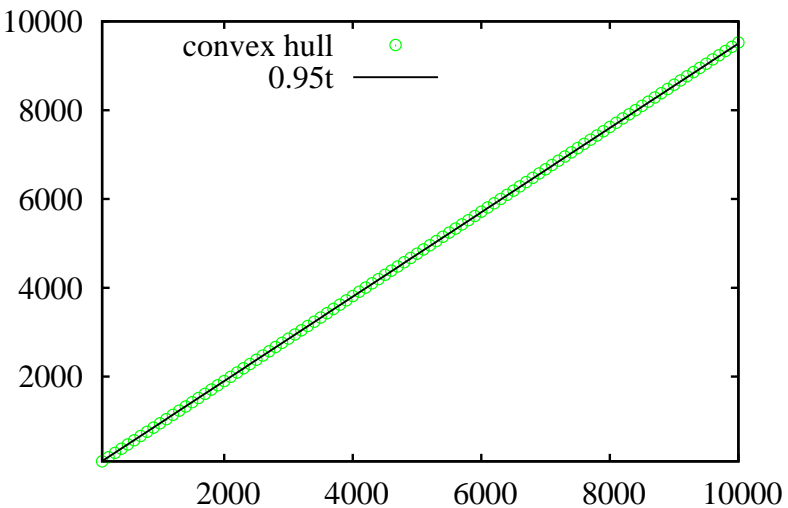

EXCESS KURTOSIS

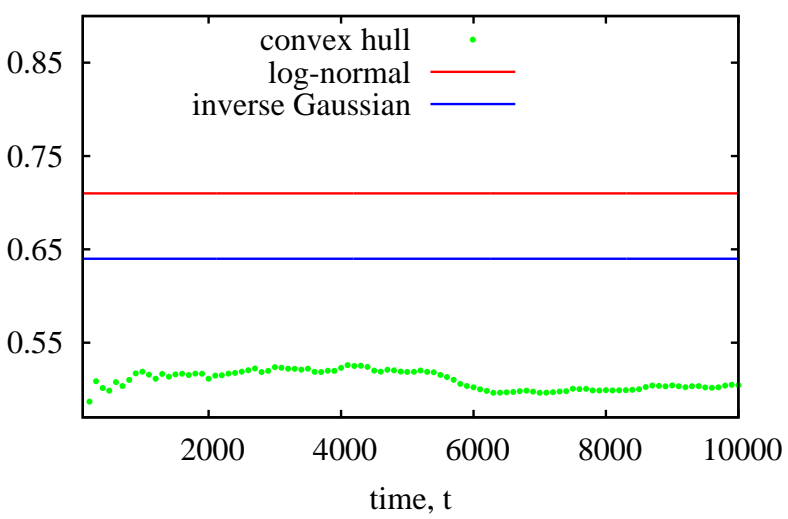

Figure 3.12: Simulation results for the time dependence of the first four standardised moments of the perimeter, $\mathbf{L}$ (green colour). In order to quantitatively demonstrate that neither the inverse Gaussian nor the log-normal distributions fit the perimeter distribution, we compare the time evolution of the respective skewness and excess kurtosis. Mean: The black curve corresponds to the analytical expression in (3.1). Variance: The black curve is a fit; the analytical expression for the variance of the perimeter is not known. The parameters $\nu, \bar{\sigma}$, $\tilde{\mu}$ and $\lambda$ were fixed so that the mean and variance of the two models coincide with the respective black curves. Bottom panels: The time dependence of the skewness and excess kurtosis of the log-normal (red) and inverse Gaussian (blue) distributions were derived using analytical expressions for the mean and variance of the perimeter (black curves). Neither the log-normal nor the inverse Gaussian moments fit well the simulated data. 
3.13). Because of the asymmetry of the shape of the random walk, the sides $a$ and $b$ of the enclosing rectangle will generally not be the same. When considered independently, the distributions $p(a)$ and $p(b)$ of the two spans will be the same. On the other hand, if we order the spans so that we always have $b>a$ (equivalent to rotating the minimum rectangle so that its longer side lies always on the same axis), then the distributions of the ordered spans will differ.

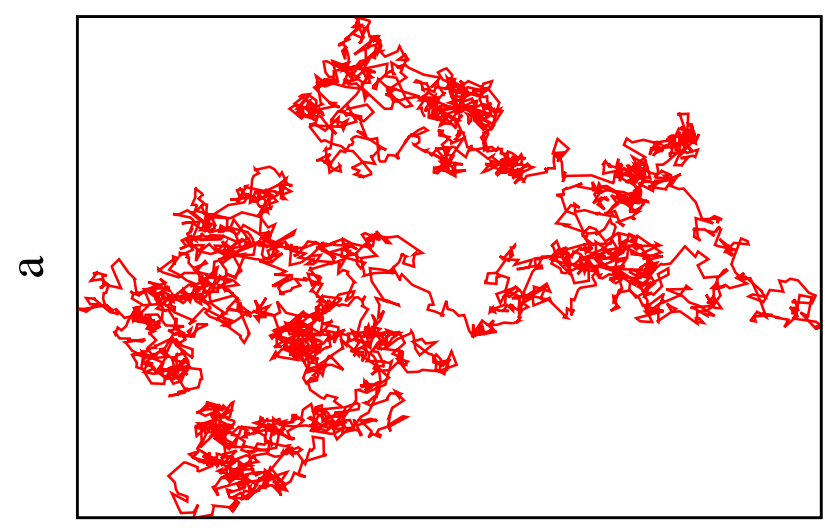

b

Figure 3.13: Span of a two dimensional random walk. When dealing with ordered spans, we will always assume that $b>a$, i.e., that the random walk is oriented such that $b$ is the longer side.

One can consider the two-dimensional span as an approximation to the convex hull. If we know what the distributions of the ordered spans are, we can determine the distribution of the perimeter and area of the minimum rectangle that encloses the entire random path. If $p_{+}(b, n)$ is the distribution of the larger spans and $p_{-}(a, n)$ the distribution of the smaller spans after $n$ jumps of the random walk, then the distribution of the length $L$ of the perimeter of the rectangle is given by

$$
p(L, n)=\int_{0}^{\infty} \mathrm{d} b \int_{0}^{b} \mathrm{~d} a p_{-}(a, n) p_{+}(b, n) \delta(L-L(a, b)),
$$

where $L(a, b)=2 a+2 b$ is the formula for the perimeter of a rectangle. Similarly, for the area we have

$$
p(A, n)=\int_{0}^{\infty} \mathrm{d} b \int_{0}^{b} \mathrm{~d} a p_{-}(a, n) p_{+}(b, n) \delta(A-A(a, b)),
$$

where $A(a, b)=a b$ is the formula for the area of a rectangle. Since it is clear that the rectangle will always overestimate the convex hull, we want to determine whether some other regular 
geometric shape approximates the hull better. We try with the ellipse and the rhombus. The area and perimeter of an ellipse are respectively

$$
A(a, b)=\frac{\pi}{4} a b
$$

and

$$
L(a, b) \simeq \frac{\pi}{2}(a+b)\left(1+\frac{3 h(a, b)}{10+\sqrt{4-3 h(a, b)}}\right)
$$

where $h(a, b)=(a-b)^{2} /(a+b)^{2}$. The area and perimeter of a rhombus are respectively

$$
A(a, b)=\frac{a b}{2}
$$

and

$$
L(a, b)=2 \sqrt{a^{2}+b^{2}} .
$$

The exact expressions for the distributions of ordered spans of a planar random have been determined by Weiss and Rubin [38]. They found that

$$
p_{-}(a, n)=2 p_{\text {span }}(a, n)\left[1-P_{\text {span }}(a, n)\right]
$$

and

$$
p_{+}(b, n)=2 p_{\text {span }}(b, n) P_{\text {span }}(b, n),
$$

where $p_{\text {span }}(r, n)$ is the distribution of the span of a one-dimensional random walk (see (3.5)) and $P_{\text {span }}(r, n)$ the corresponding cumulative distribution function.

Because of the non-trivial nature of 3.5 it is necessary to determine the integrals in 3.11 and 3.12 numerically. The results are shown in figure 3.14 from which we can deduce that neither an ellipse nor a rhombus can be used as a good approximation to the convex hull.

However, a satisfactory data collapse is obtained for the various geometric shapes using the following rescaled forms

$$
p(L, n)=\frac{1}{\sqrt{\operatorname{Var}[L]}} \Phi_{L}\left(\frac{L-E[L]}{\sqrt{\operatorname{Var}[L]}}\right)
$$

and

$$
p(A, n)=\frac{1}{\sqrt{\operatorname{Var}[A]}} \Phi_{A}\left(\frac{A-E[A]}{\sqrt{\operatorname{Var}[A]}}\right)
$$

where $\operatorname{Var}[\cdot]$ and $E[\cdot]$ are the variance and the mean respectively, both of which depend on $n$.

According to figure 3.15 the rescaled perimeter distributions of the rhombus and ellipse (obtained by numerical integration) fit remarkably well with the rescaled perimeter distribution of the convex hull (obtained by numerical simulations). This, however, is not the case for the rectangle. In the case of the area distribution, it is no surprise that the scaling functions are the same for the ellipse and the rhombus since the formulas for the area differ by a factor only. For completeness, in figure 3.16 we show how the scaling functions of the various span distributions compare with the scaling function of the perimeter of the convex hull. 

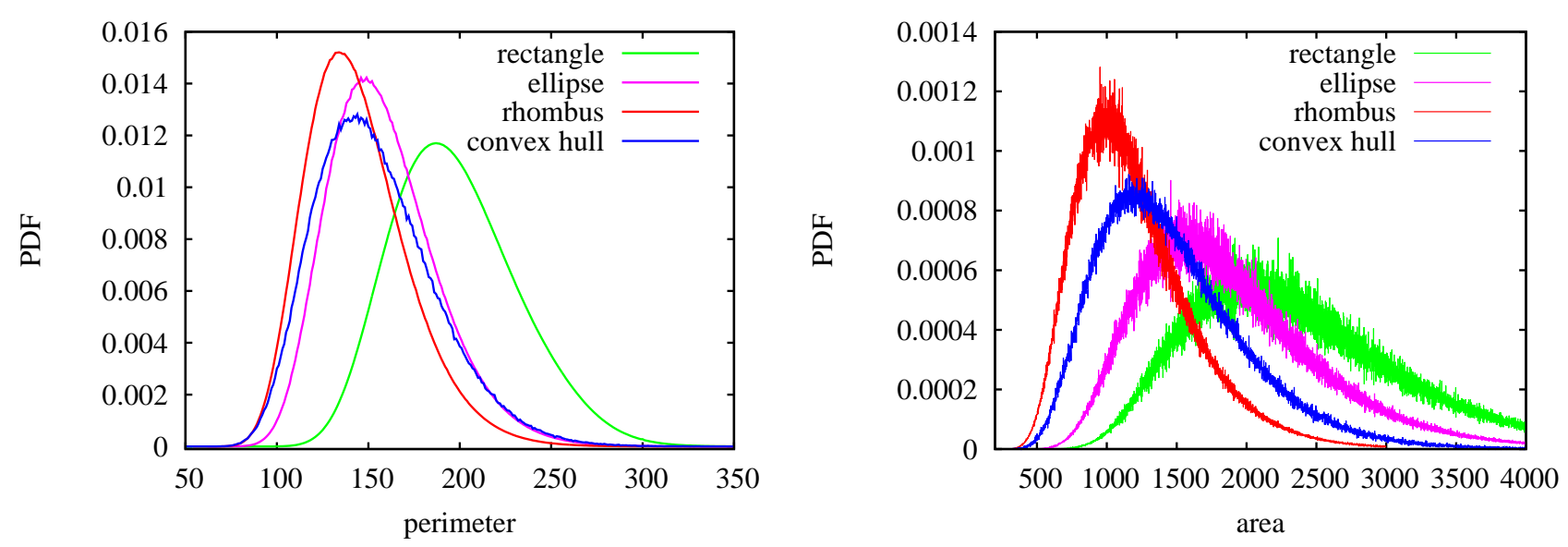

Figure 3.14: Perimeter and area of different geometric shapes compared to that of the convex hull. The underlying planar random walk has $\sigma=\sqrt{2}$ and $n=1000$ steps. The left (right) panel shows the probability distribution of the perimeter of the corresponding convex hull and how it compares to the perimeter (area) distribution of the rhombus and the ellipse models.
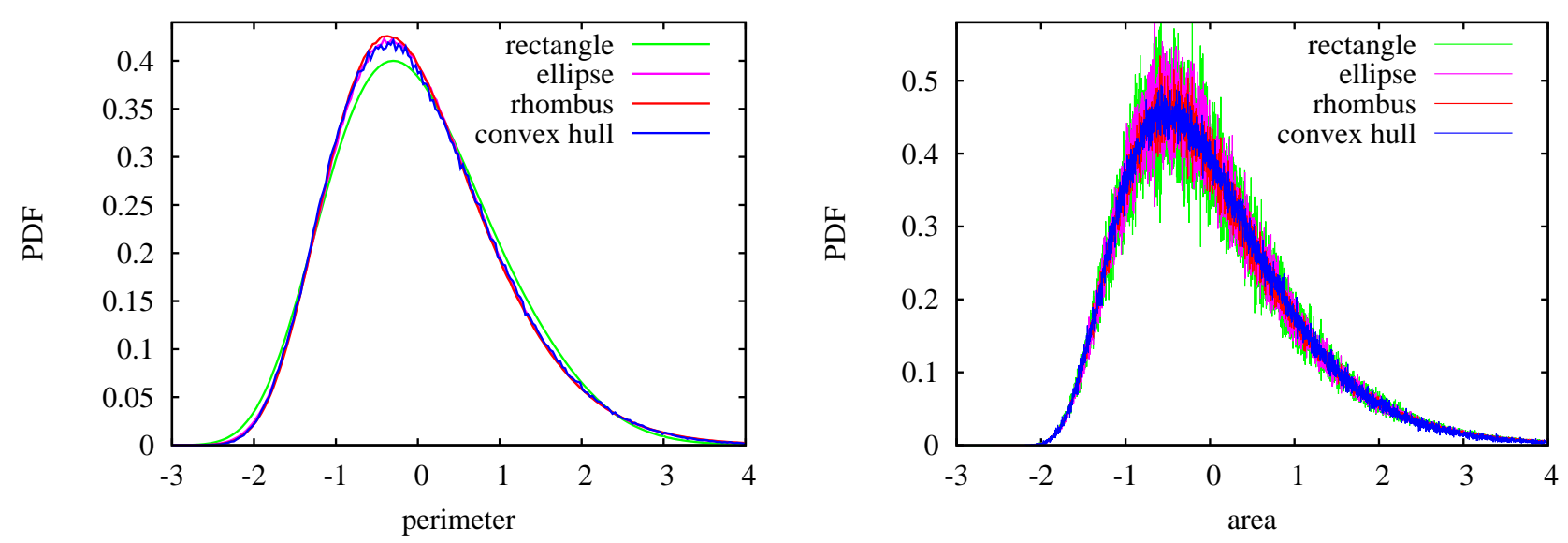

Figure 3.15: Distribution of the rescaled perimeter (left panel) and the rescaled area (right panel) for different geometric objects. The figure shows the functional form of $\Phi_{L}(\tilde{L})$ (left panel) and $\Phi_{A}(\tilde{A})$ (left panel) for the three different geometric shapes and the convex hull. The rescaled perimeter and area are respectively $\tilde{L}=(L-E[L]) / \sqrt{\operatorname{Var}[L]}$ and $\tilde{A}=(A-E[L]) / \sqrt{\operatorname{Var}[A]}$. 


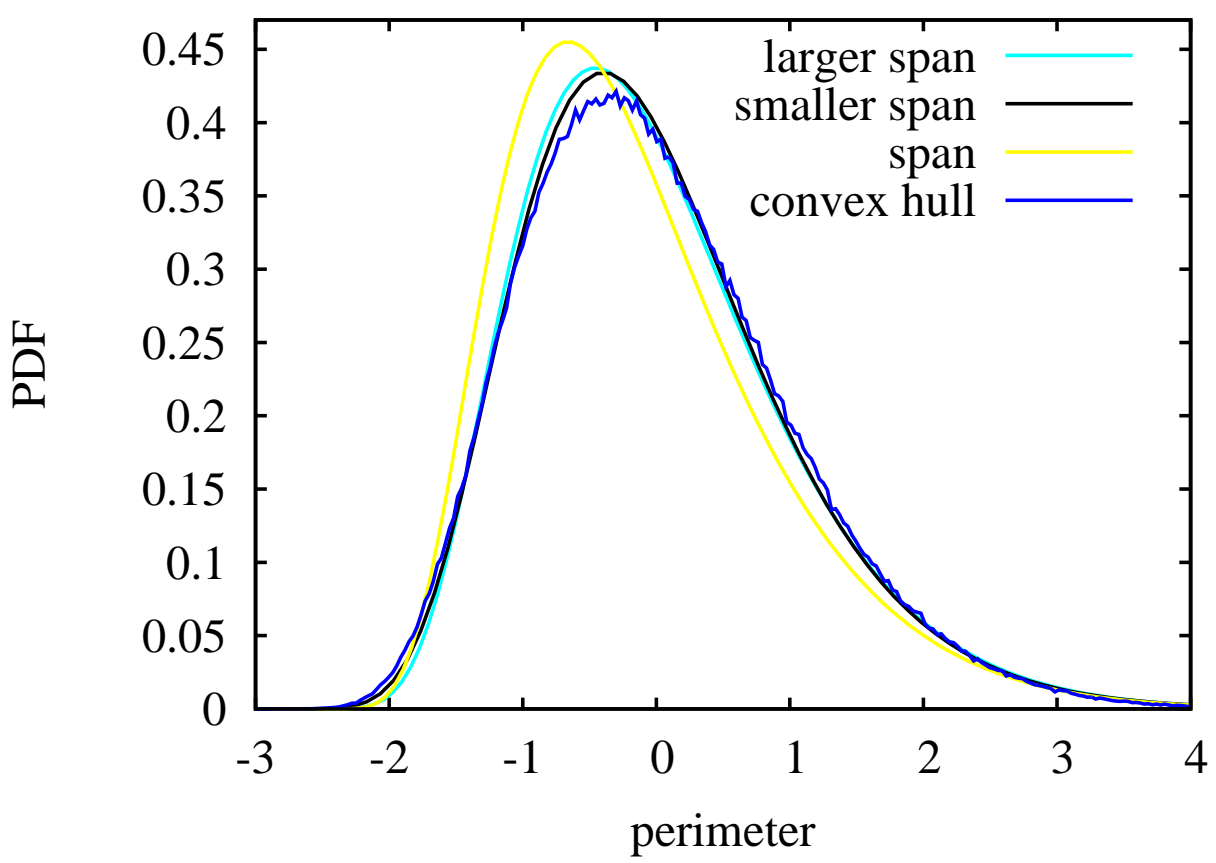

Figure 3.16: Scaling function of the different span distributions compared to the scaling function of the perimeter of the convex hull. 


\subsection{Convex Hulls of Lévy Walks}

In the previous chapter, we already described the Lévy walk as a model for super-diffusion whereby the walker moves with constant velocity $v$ between the turning points of its trajectory (see figure 3.17). The space-time coupling present in the process implies that the random waiting times and jump lengths are not independent from one another. The jump probability density has the conditional form $\psi(\Delta x, \tau)=\eta(\Delta x \mid \tau) \psi(\tau) \propto \delta(|\Delta x|-v \tau) \psi(\tau) / 2$, so that a jump of a certain length always involves a time cost. The finite velocity ensures that the walker can only travel a maximum distance in a finite time interval.

Super-diffusion is obtained by assuming that the waiting time distribution $\psi(\tau)$ follows a heavy-tailed power law $\psi(\tau) \propto \tau^{-\alpha-1}$ with $0<\alpha<2$. In case $\alpha \geq 2$ or more generally, if $\psi(\tau)$ is a distribution with a finite second moment, then the resulting process is equivalent to a simple random walk and the resulting diffusion is normal. We shall reserve the term Brownian walk for this case.
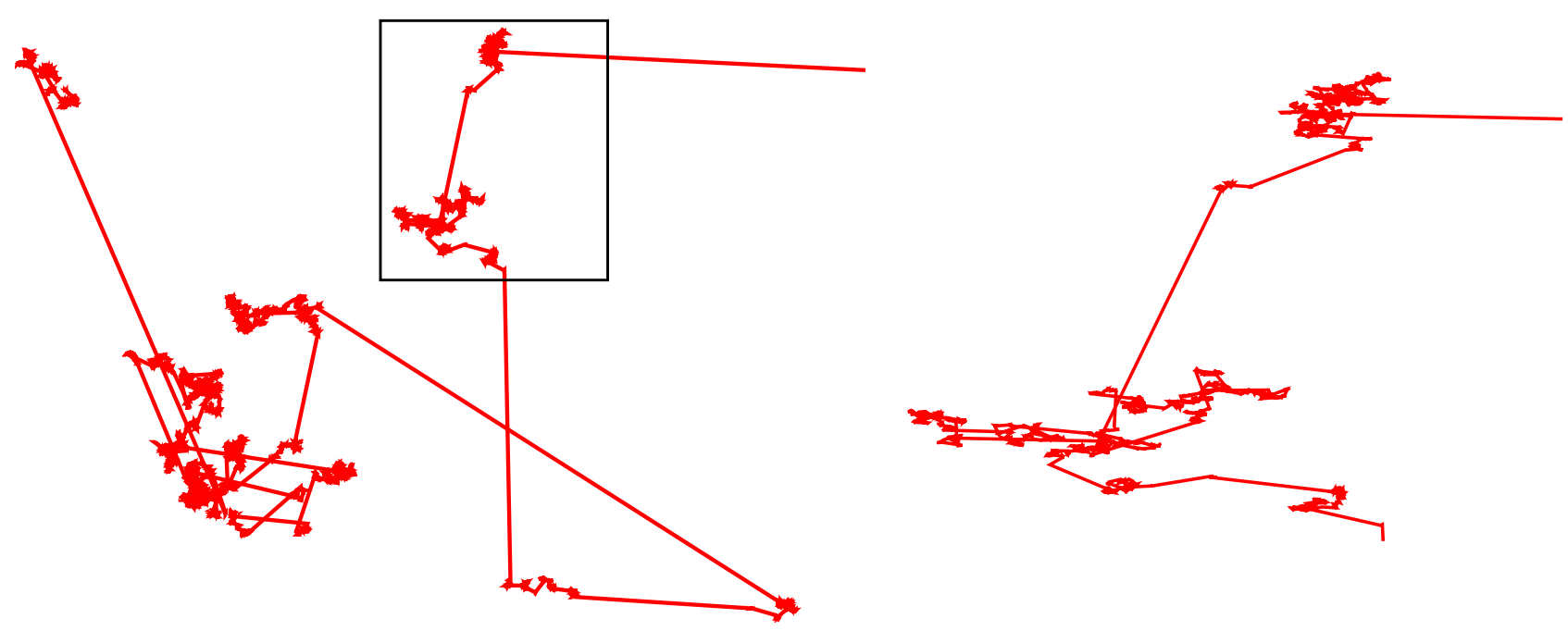

Figure 3.17: A typical trajectory of a Lévy walk. It is characterised by frequent long excursions of different length scales that produce a self-similar process. The trajectory in the right panel is part of the trajectory in the left panel enclosed by the box. They are identical from a statistical point of view, a hallmark of self-similarity.

The propagator $p(x, t)$ of the Lévy walk is a symmetric distribution that vanishes for $|x|>v t$ because of the cut-off introduced by the space-time coupling. Furthermore, it has two different forms, depending on whether $0<\alpha<1$ or $1<\alpha<2$. In the former case it has a minimum in the centre and singularities at $x= \pm v t$, much like the arcsine distribution. In the latter case, the propagator can be divided into characteristic domains: A central part with a Gaussian form, power law decaying flanks and two $\delta$-function or ballistic peaks at the extreme ends where $x= \pm v t$. The ballistic peaks represent the random walkers that maintain the same direction upto the observation time $t$. The number of such rectilinear walkers decreases with time causing 
the peaks to decay according to $t^{1-\alpha}[50]$.

When $0<\alpha<1$, the mean square displacement of the Lévy walk is quadratic in time and therefore the diffusion is said to be ballistic. On the other hand, when $1<\alpha<2$, in the asymptotic time limit, the mean square displacement grows as $t^{3-\alpha}[16]$. Since we are considering a diffusive random foraging model, the ballistic case will be ignored.

Since the convex hulls of Lévy walks are even more difficult to study than their random walk counterpart, we will use numerical simulations and heuristic methods to analyse their properties. We begin with the probability distributions of the perimeter and area of the Lévy hull. Figures 3.19 and 3.21 show the probability distribution and the complementary cumulative distribution function of the perimeter $L$ and the area $A$ for different values of the stability parameter $\alpha$. The results correspond to computer simulation in which many Lévy walk trajectories of duration $T=1000$ were generated after which their respective convex hulls were determined and perimeter and area calculated. The use of the complementary cumulative distribution helps reduce the noise that would otherwise be present in the tail of the probability distribution, usually making it difficult to observe the power-laws.

A closer inspection of figure 3.19 reveals that there is an abrupt transition from a rapidly decaying distribution when $\alpha=2$ to a broad-tailed distribution when $\alpha<2$. This comes as no surprise since the underlying random process becomes scale-free as soon as $\alpha$ becomes smaller than two.

A very useful concept is the scaling property since it enables us to obtain important insights and results without knowledge of the exact form of the probability density. Formally, when we talk about scaling, it meaningful only in the context of a probability distribution $p(x, t)$ and we can express the scaling property using the equation

$$
p(x, t)=\frac{1}{t^{\delta}} \Phi\left(x / t^{\delta}\right),
$$

where $\Phi$ can be any normalisable function. For a random walk $\delta=1 / 2$ and in the asymptotic time limit $\Phi$ is the Gaussian distribution. From the above expression we conclude that $x(t) \propto t^{\delta}$, i.e. the length evolves with time according to $x \propto t^{\delta}$.

In the particular case of a Lévy walk with $1<\alpha<2$, the overall distribution $p(x, t)$ does not scale as a whole [51]. Instead, the Lévy walk is characterized by a bimodal scaling [52]. The ballistic peaks scale with $\delta=1$, while the rest of the distribution scales with $\delta=1 / \alpha$.

If we assume that asymptotically in time, the perimeter $L(T)$ is a linear function of $x(T)$ and that the area $A(T)$ is a linear function of $x^{2}(T)$, i.e. $L \sim x$ and $A \sim x^{2}$, then for the evolution of the average values of the perimeter we have

$$
\begin{aligned}
\langle L(T)\rangle \sim \int_{0}^{\infty} x p(x, T) \mathrm{d} x & \simeq \int_{0}^{\infty} x \frac{1}{T^{1 / \alpha}} F\left(\frac{x}{T^{1 / \alpha}}\right) \mathrm{d} x+\int_{0}^{\infty} x T^{1-\alpha} \delta(x-T) \mathrm{d} x \\
& =c_{1} \cdot T^{1 / \alpha}+c_{2} \cdot T^{2-\alpha}=\mathcal{O}\left(T^{1 / \alpha}\right),
\end{aligned}
$$

where $c_{1}$ and $c_{2}$ are constants. When considering the average area, the ballistic peaks of the Lévy walk do not contribute because they correspond to a Lévy walker that does not change its 
direction during the entire observation time $T$. Therefore, the trajectory and the convex hull will be one-dimensional lines, with zero area. Consequently, for the evolution of the average area we have

$$
\langle A(T)\rangle \sim \int_{0}^{\infty} x^{2} p(L, T) \mathrm{d} x \simeq \int_{0}^{\infty} x \frac{1}{T^{1 / \alpha}} F\left(\frac{x}{T^{1 / \alpha}}\right) \mathrm{d} x=c_{3} \cdot T^{2 / \alpha}=\mathcal{O}\left(T^{2 / \alpha}\right),
$$

where $c_{3}$ is a constant. Notice that in both cases the integral is over the positive domain $[0, \infty]$ since $L$ and $A$ are strictly positive variables. Figure 3.18 shows numerical results for the evolution of the average perimeter and average area for different values of the stability parameter $\alpha$ (different shades of red). The black curves correspond to the theoretical prediction based on the scaling arguments in (3.22) and (3.23); the figure shows good agreement between the two.

The two scaling regimes present in the Lévy walk propagator suggest that this property will be conveyed to the convex hull. We therefore use the two scaling regimes separately on the perimeter and area distributions of the convex hull in order to determine whether there is a data collapse. The results are shown in figures 3.20 and 3.22.
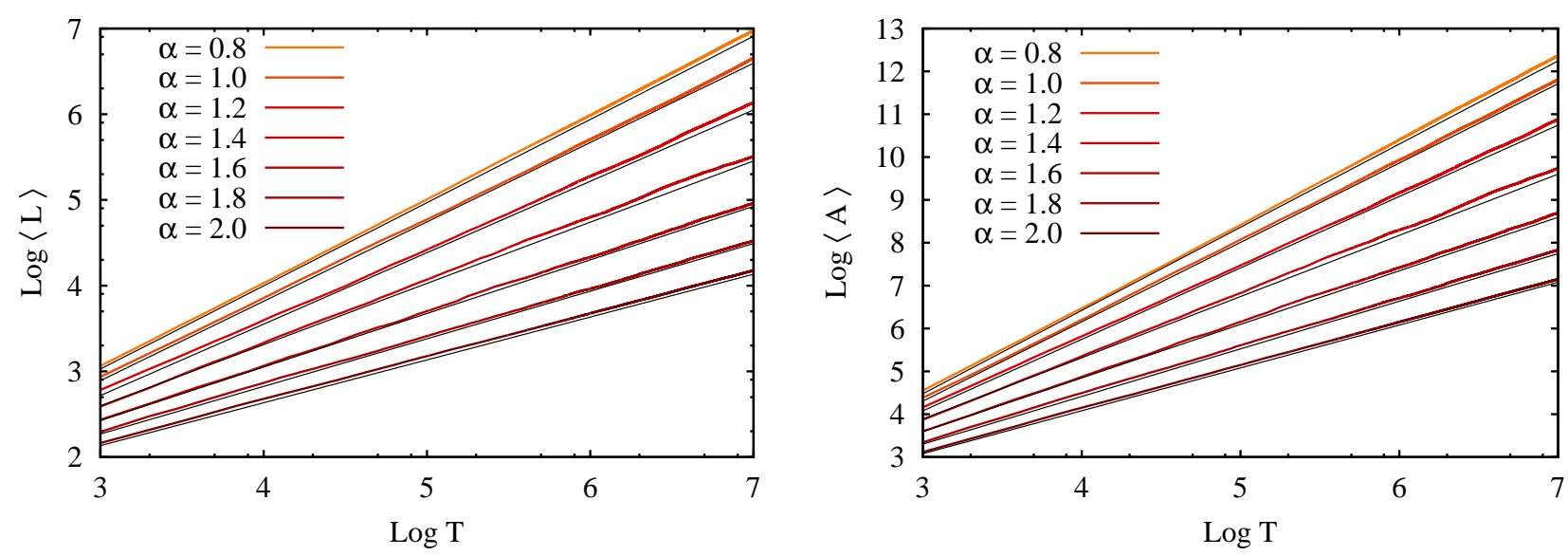

Figure 3.18: Time evolution of the average perimeter (left panel) and average area (right panel) of the convex hull of a Lévy walk with different stability parameters $\boldsymbol{\alpha}$. The black lines correspond to the functions $c_{L} T^{1 / \alpha}$ (left panel) and $c_{A} T^{2 / \alpha}$ (right panel) derived for the average values using scaling considerations (see equations (3.22) and (3.23)). The constants $c_{L}$ and $c_{A}$ depend on $\alpha$.

Since we want to study the properties of genuine Lévy walks that are space-time correlated, we construct the sample trajectories numerically as a function of a time parameter $T$ indicating the duration of the trajectory and not as a function of the number of steps.

There exist other ways to reduce the statistical errors in the power-law tail, for example by choosing logarithmically increasing bin sizes [53]. Here, we focus on studying cumulative distributions as they carry substantial information on the nature of the stochastic processes under study. In particular, we use the "survival probability" instead of the normal cumulative 
distribution function (CDF) because the latter does not show the broad tail if it exists and therefore the shape of the curve changes only slightly when analysing different types of random walks.
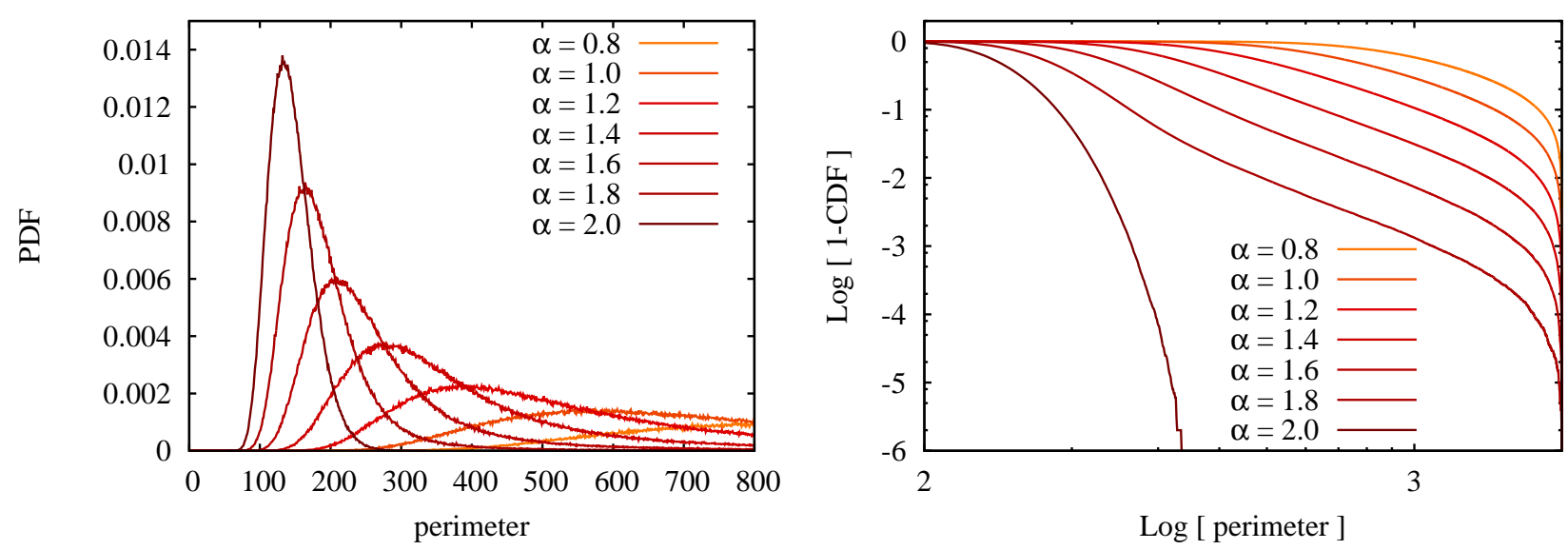

Figure 3.19: Probability distribution of the perimeter length, L of the convex hull of a Lévy walk with different values of the stability parameter $\alpha$. The total walk time is $T=1000$ units. The left panel shows the probability density and the right panel shows the complementary cumulative distribution function.

\subsection{Discriminating between normal and anomalous dif- fusion}

Recently there has been much debate in the scientific community as to whether the observed walk patterns of foraging animals are Lévy-like or not. This is mainly due to the poor accuracy of the statistical methods employed to identify Lévy behaviour from collected data samples of animal trajectories $[53,11,54]$. Since ideal power laws rarely exist in nature, current methods rely on accurately estimating stability parameter $\alpha$ of a truncated power law. There are many ways in which Lévy walks can be identified from data. The simplest one consists of collecting all the straight paths between relocation points into a histogram. If the plotted data set is well fitted by a straight line in a log-log scale, then it might be reasonable to conclude that the underlying random walk is indeed a Lévy walk. However, there are several drawbacks with such a simple approach, especially when the data set under examination is unreliable or small $[10,53,11]$. Another popular and well established method consists of analysing the jump lengths using the maximum likelihood estimates for Lévy parameters $[11,55]$. However, it has been shown that in some cases the maximum likelihood estimation method can give false positives and other pitfalls with regard to Lévy walks [9]. 

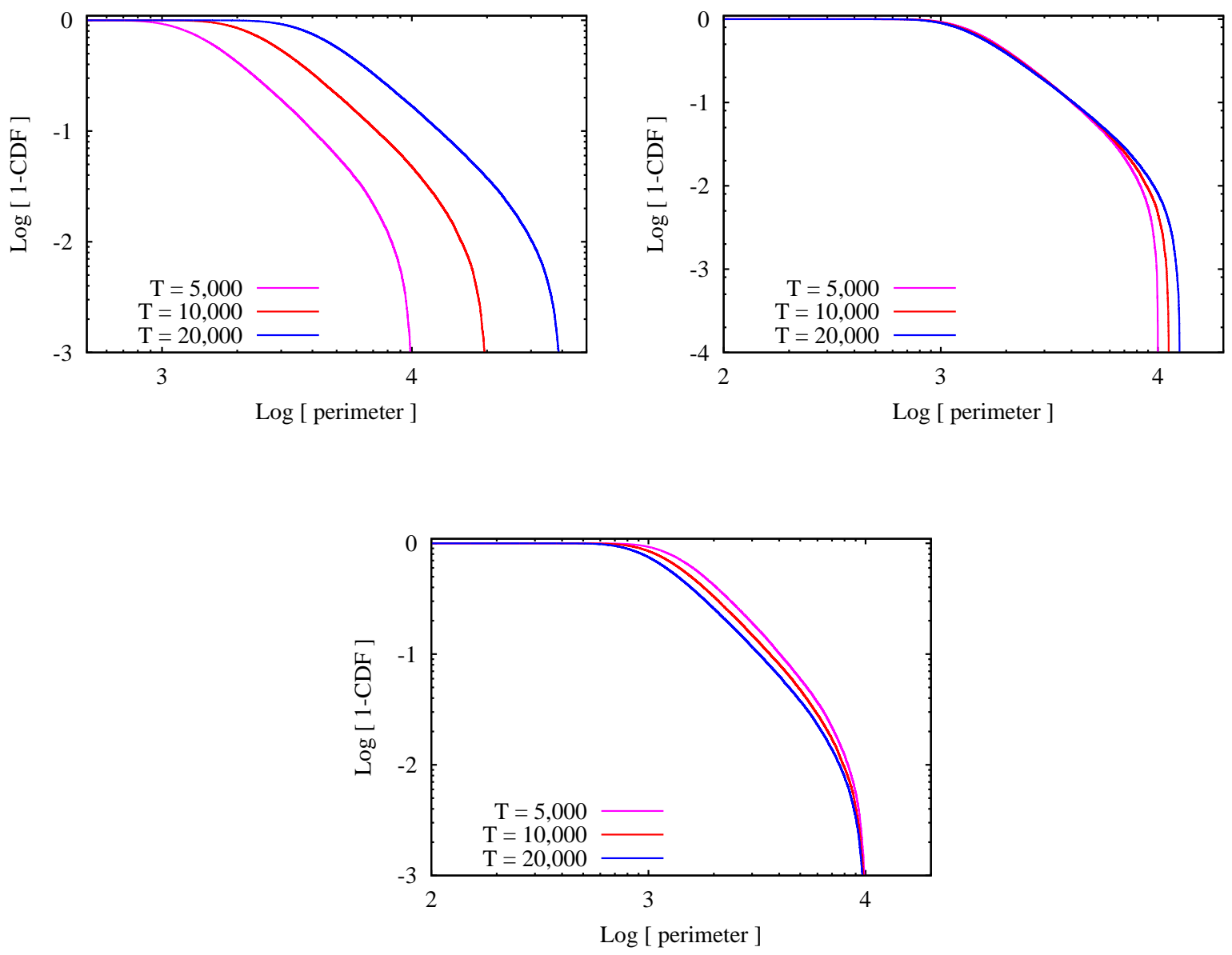

Figure 3.20: Partial collapse for the perimeter, $\boldsymbol{L}$. The results presented in this figure were obtained using Lévy walks with $\alpha=1.2$ for three different time intervals T. Top left: complementary cumulative distribution function of the perimeter. Top right: complementary cumulative distribution function for the rescaled perimeter $L / T^{1 / \alpha}$. Bottom: complementary cumulative distribution for the rescaled perimeter $L / T$. The partial collapse can be attributed to the bimodal form of the propagator $p(x, t)$ of the underlying Lévy walk. In the bottom panel, when we use the ballistic scaling $(\delta=1)$, only the tails of the distributions collapse. This is because the tails correspond to Lévy walkers that never change their direction. On the other hand, the Lévy scaling $(\delta=1 / \alpha)$ in the top right panel results in the collapse of the central parts of the distributions, which is related to the decaying power law flanks of the Lévy walk propagator. 

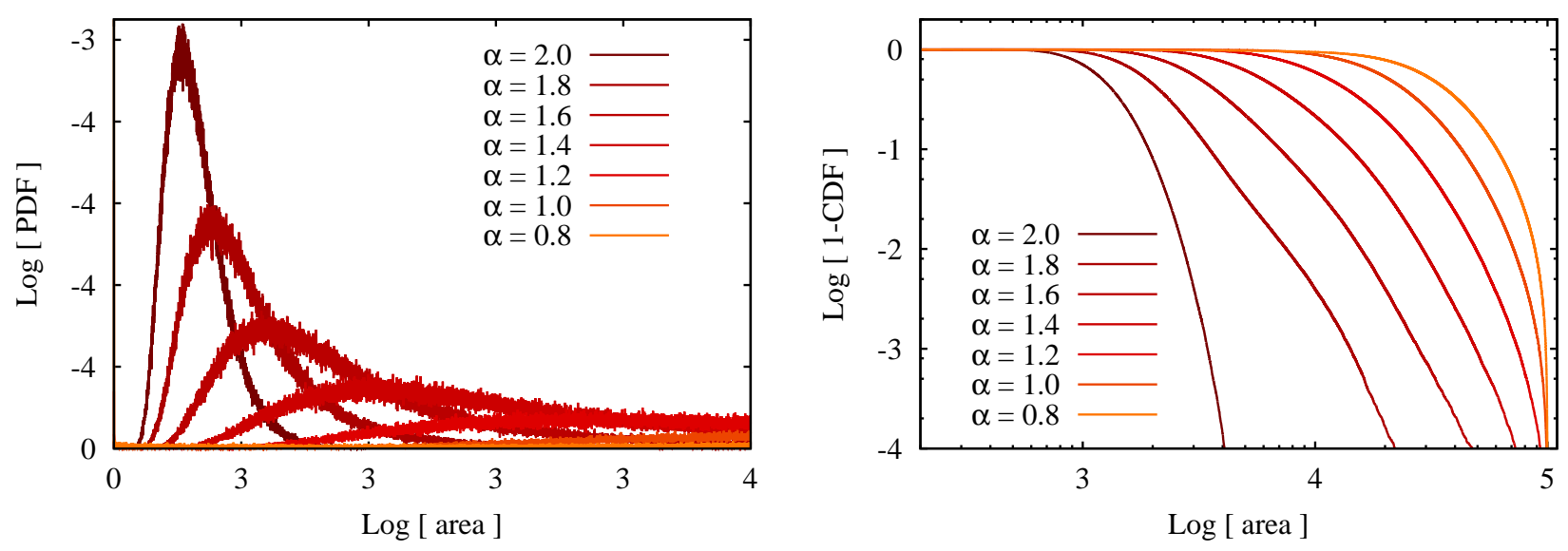

Figure 3.21: Probability density of the area A of the convex hull of a Lévy walk for different values of the stability parameter $\boldsymbol{\alpha}$. The total walk time is $T=1000$ units with $\alpha$ ranging from 0.8 to 2 (from orange to dark red) in steps of 0.2.
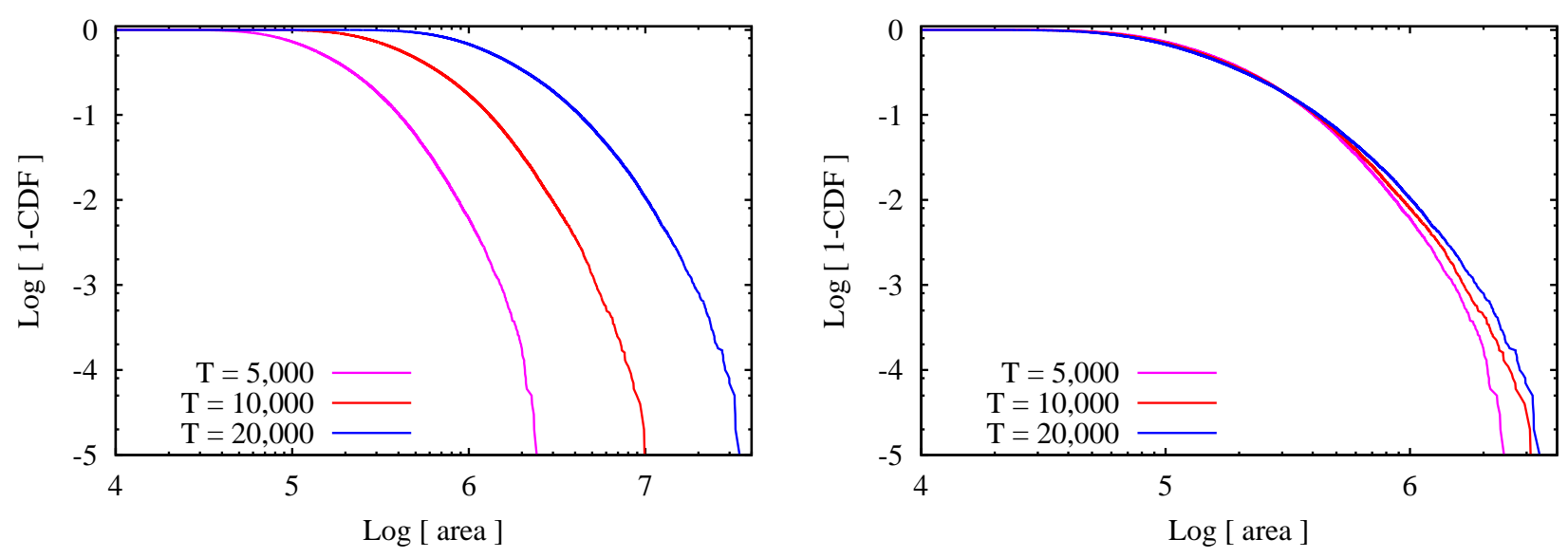

Figure 3.22: Partial collapse for the area, $\boldsymbol{A}$. The results presented in this figure were obtained using Lévy walks with $\alpha=1.2$ for three different time intervals $T$. Left: complementary cumulative distribution function of the area. Right: complementary cumulative distribution function for the rescaled area $L / T^{2 / \alpha}$. Since the area does not depend on the $\delta$-function peaks of the Lévy walk propagator, we do not expect a collapse for the corresponding ballistic scaling. 
We propose the use of convex hulls of the home range of animals as a robust and accurate way of discriminating between normally diffusive processes and those exhibiting anomalous diffusion. The method is simple and robust even in the case where data available is sparse and noisy. Since there is no need to reconstruct the trajectory, knowledge of the temporal order of the visited locations is not required.

Special care is required when attempting to determine whether a random walk is truly Lévylike. Observing a Lévy type pattern does not necessarily mean that the underlying process is a Lévy walk. For example, a composite or a correlated random walk can resemble a Lévy walk over short time scales. In such cases the mean square displacement may appear to be non-linear in certain time intervals despite the fact that the jumps are Gaussian distributed [56]. The super-diffusive behaviour is induced by the strong correlations in the direction of movement.

An ambitious goal would be to develop the convex hull method to be used by ecologists on real data. Here, we present a first step toward this goal. In particular, we focus on the analysis of artificially generated data and show that the method works under controlled conditions, which is a necessary requirement before using it on real data. Therefore, when we refer to data or data sets we mean a set of coordinates of numerically created points in the plane that are intended to resemble a set of visited locations. The points in the dataset are sampled from a simulated planar random process that starts at time $t=0$ and ends at time $t=T$. The absence of a time stamp leaves us only with a cloud of points or point-cloud in the plane emulating the points visited by the animal during a time interval of total duration $T$, which is usually the observation time or the time of data acquisition.

In cases where it is possible to attach a transponder or telemetry tag to an animal the coordinates of the trajectory are ideally obtained at regular time intervals. Depending on the sampling rate and the precision with which the position of the animal can be located, it is possible to reconstruct the trajectory of the animal. On the other hand, if a forager cannot be tracked via telemetric methods, field researchers have to rely on direct observation or on traces left behind in the environment for data collection. In such cases it might not be possible to determine when the forager occupied a certain location and the reconstruction of the most likely trajectory is often extremely difficult. For our method based on convex hulls, in contrast, temporally resolved information is not required.

Instead, we shall rely on ensembles of convex hulls of independent and statistically identical point-clouds. By statistically identical it is understood that each point-cloud is the result of sampling the same type of random process for the same time period $T$ with the same starting location, the origin (figure 3.23).

The advantage of using convex hulls for identifying Lévy-type processes are manifold. First of all, as shown earlier, it is straightforward to determine the convex hull of a set of points using one of the available algorithms. Furthermore, convex hulls depend strongly only on the extreme points, the ones on the periphery of the point cloud. Consequently, it is very robust to 


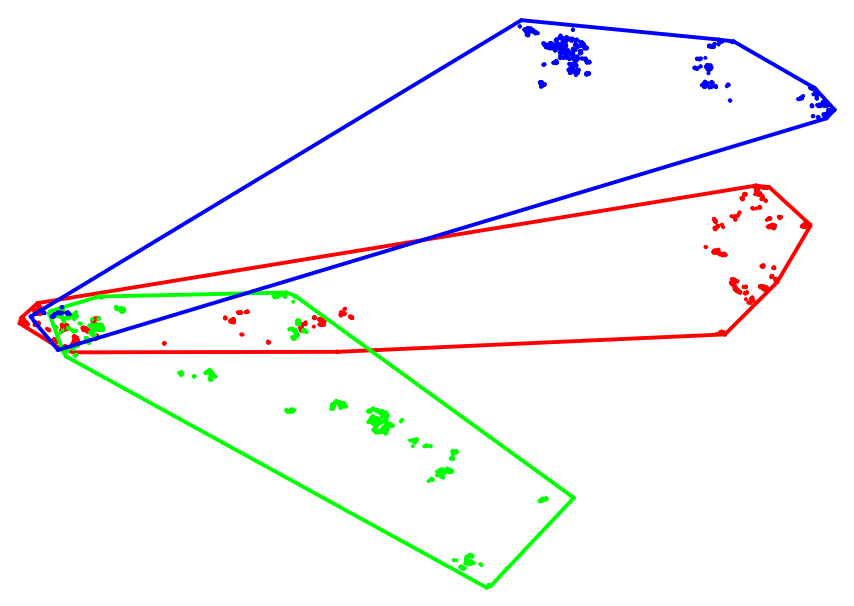

Figure 3.23: Example of the numerical data used. Shown are three statistically identical and independent point-clouds of a Lévy walk with $\alpha=1$ in the time interval $[0, T]$.

random changes in the position of the points of the underlying trajectory. If, for example, we remove a large fraction of the points from the data set, it is still possible to distinguish between random walks and Lévy walks (see figure 3.31).

Since data acquisition and processing can be rather complex, it is desirable to have a method for analysis that requires very little or no data preparation at all. Very often the recorded tracking signal is intermittent, providing an irregular sampling rate. This might be due to a temporary malfunction of the transmitting device caused by atmospheric humidity, temperature or simply by the abrupt movement of the animal. Even when the transmitting device functions perfectly, the signal might not reach the receiver because of bad weather or interferences from other radio-sources. As a consequence, the resulting data set might be very difficult to interpret. Most importantly, it might be difficult to identify the points at which the animal changes its direction of movement (see figure 3.24) [9]. In that case, a meaningful histogram of the distances between consecutive relocation points becomes an arduous task. It is in cases such as these that the convex hull method may substantially outperform traditional methods. Furthermore, Codling and Plank showed that misidentifying Lévy walks may even be due to the sampling method and the turn designation process rather than the inherent properties of the underlying random walk $[9,57]$.

In the algorithm that we propose, it is not required to identify the turning points and the sampling method does not affect considerably the result (see figure 3.31). Finally, the method also works well when the data set is without a time-stamp so that we do not need to know in which order the points in the data set were visited by the foraging animal. 


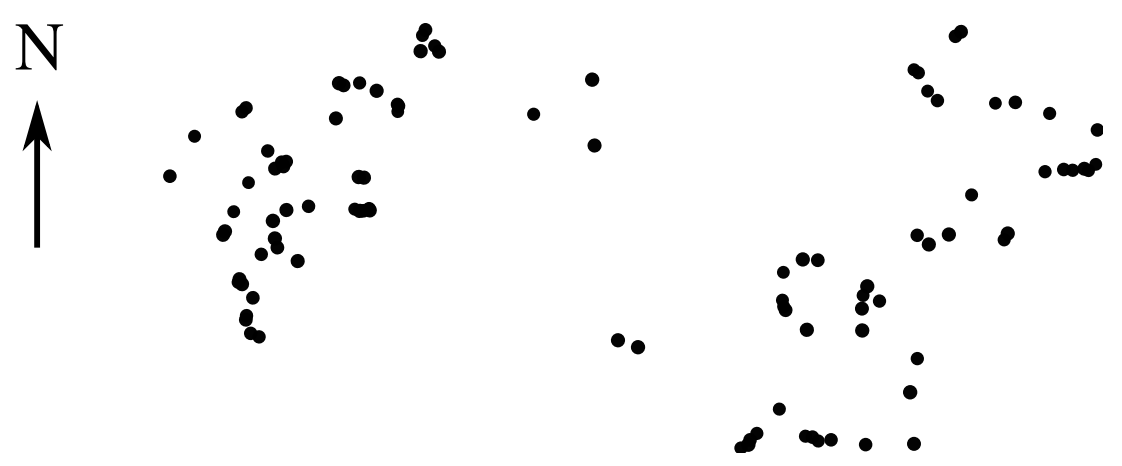

Figure 3.24: A hypothetical point-cloud obtained by sampling a planar random process such as foraging. An underlying process that is a Lévy-like will leave behind a trail that appears patchy due to its scale-free nature. In this case the point-cloud is also known as Lévy dust (see figure 3.23). Knowing the order in which the points were visited simplifies the task of reconstructing the trajectory of the random walker. However, it might still not be possible to determine which subset of points are the relocation points [9, 58].

If the data set is abundant, the complementary cumulative distribution functions of the perimeter and area of the convex hull often give an indication as to whether the underlying random process is truly Lévy-like. In figure 3.19 the rapidly decaying complementary cumulative distribution function that corresponds to a normal Brownian walk $(\alpha \geq 2)$ is easily distinguishable from the slowly decaying distribution that corresponds to a Lévy walk $(\alpha<2)$. However, the situation is more complicated for small data sets in which case the survival probability might be difficult to interpret. For example, figure 3.25 shows that for an ensemble of 100 independent Lévy walk trajectories of the same time duration, the form of the complementary cumulative distribution function associated with the correlated random walk is very similar to the one associated with the Lévy walk; the two curves have very similar shapes and cannot be distinguished. The correlated random walk can display super-diffusive behaviour over short time windows because of the presence of persistence in its direction of movement and can therefore easily be misidentified as a Lévy walk.

We propose a far more reliable measure, the ratio $\chi$ between the perimeter of the hull and its area. It performs substantially better in distinguishing Lévy walks from various other types of random processes. In order to discriminate between Lévy walks and other types of "non-anomalous" processes, we propose the following algorithm:

1. Prepare an ensemble of $N$ independent point-clouds, each collected over a fixed time period $T$.

2. Determine the convex hull of each point cloud in the ensemble.

3. Calculate the perimeter $L$ and area $A$ of each convex hull. 

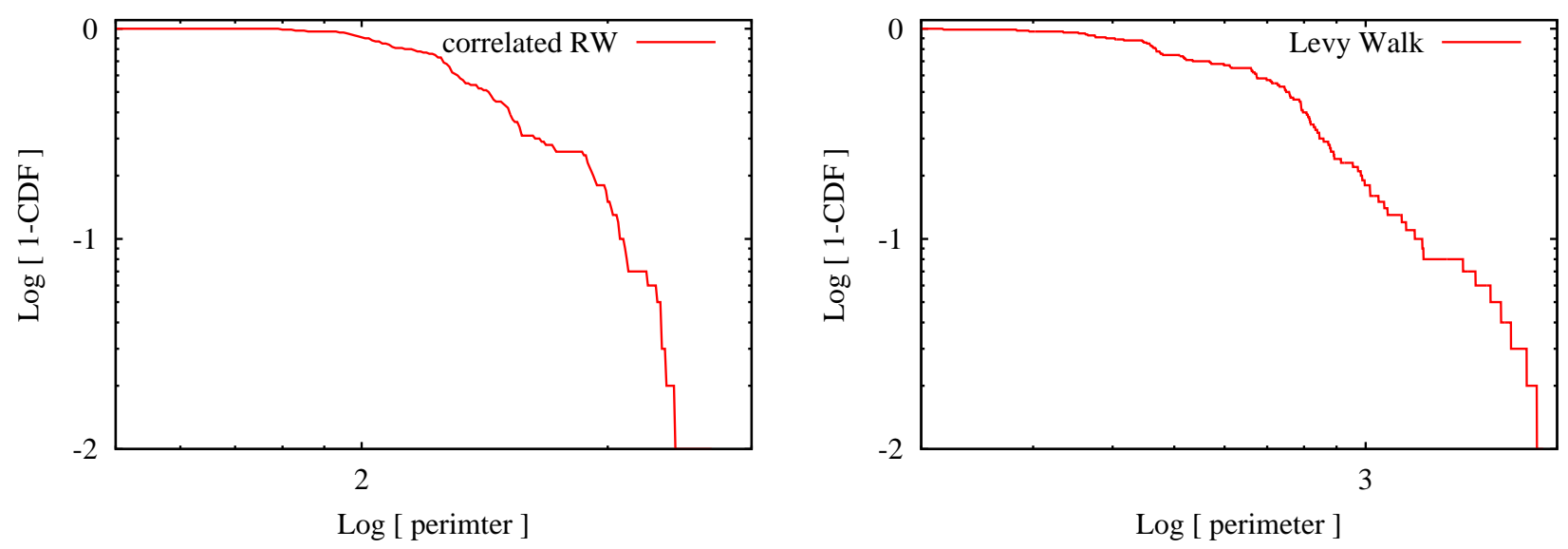

Figure 3.25: Correlated random walk vs. Lévy walk - comparison of complementary cumulative distribution functions. Shown is the correlated Brownian walk of duration $T=1000$ and $\sigma=\sqrt{2}$ (left panel) and a Lévy walk with $\alpha=1$ and a duration of $T=1000$ units (right panel). In both cases we have used an ensemble size of 100 point cloud sets. By comparing the shapes of the two curves, it is very difficult to distinguish one from the other.

4. Use the perimeter and area to construct a histogram $P(\chi)$ of their ratio $\chi=L / A$.

5. Integrate the histogram in order to obtain the complementary cumulative distribution function

$$
F(\chi)=\int_{\chi}^{\infty} P(x) d x=1-\int_{0}^{\chi} P(x) d x .
$$

The results of applying the algorithm to data sets that correspond to Lévy walks with different stability parameters $\alpha$ are shown in figure 3.26. Each curve in the figure is the result of a single application of the algorithm. As was explained earlier, the use of the complementary cumulative distribution helps reduce the noise that would otherwise be present in the tail of the probability distribution, usually making it difficult to observe the power-laws. The first important fact that can be observed from the figure is that the curves corresponding to $\alpha<2$ are comprised of two parts, a rapidly decaying regime for smaller values of $\chi$ and a slowly decaying power law tail. As $\alpha \rightarrow 2$ the tail gets smaller and smaller until it finally vanishes leading to the second important fact, that the Brownian path can be identified by a rapidly decaying curve without the power law tail. Based on this result, we can state that Lévy type walks can be identified by the presence of a power-law tail in the function $F(\chi)$ and that normal planar random walks can be identified by the absence of the broad tail. The extent of the power law tail could in principle be used as a measure of how Lévy-like the trajectory is. However, the most important prerequisite for applying this method is the availability of large data sets.

The crucial ingredient of the algorithm is the convex hull, so it is irrelevant whether the 
points in the data have a time stamp or not. What is important is that there exists an ensemble of statistically identical and independent point-clouds.
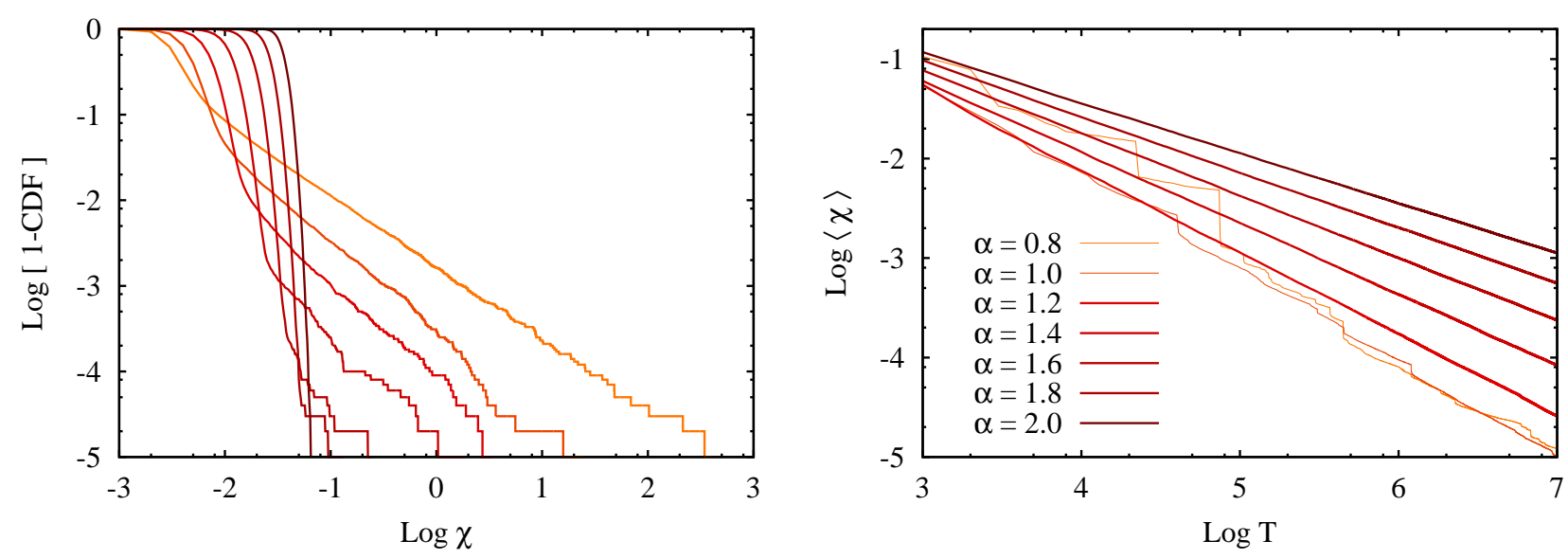

Figure 3.26: Properties of $\chi=\boldsymbol{L} / \boldsymbol{A}$. Left: The complementary cumulative distribution function of $\chi$ corresponding to Lévy walks with different stability parameters $\alpha$. The total walk time is $T=10000$ units with $\alpha$ ranging from 0.8 to 2 (from orange to dark red) in steps of 0.2. Each ensemble is made of $10^{5}$ point-clouds. Right: Time dependence of the $\langle\chi\rangle$. For the scaling of the complementary cumulative distribution function of $\chi$ see figure 3.27.

The key question is how reliable this method is. The amount of data used to produce figure 3.26 is rather unrealistic. What happens when it is considerably smaller and when we use modified versions of the random walk, such as the composite random walk or the correlated random walk? These are usually much harder to distinguish from Lévy walks. We have to ensure that the algorithm identifies them as processes displaying normal diffusion.

In his 2007 paper, Benhamou considers a the composite random walk, which is a mixture of two normal random walks, whereby one is made of sporadic exponentially distributed steps with a large mean and the other made of much more frequent exponentially distributed steps with a small mean [8]. With a simple numeric example, it is shown that the likelihood for false positives is often high, meaning that a composite Brownian walk is often confused with a Lévy walk when using step length frequency distributions with a linear binning to interpret the data.

Figure 3.28 shows the results of applying the algorithm to four different types of random processes, three displaying normal diffusion (normal random walk, composite random walk, correlated random walk) and one super-diffusive (Lévy walk). The correlated random walk is such that its $i^{\text {th }}$ jump is at an angle $\theta_{i}=\theta_{i-1}+\theta_{\varepsilon}$, where $\theta_{\varepsilon}$ is a very small angle sampled from a uniform distribution centred around zero. The composite random walk that very frequently makes jumps of a smaller length scale and from time to time makes jumps of a much larger scale.

The complementary cumulative distribution curves that correspond to the three processes 

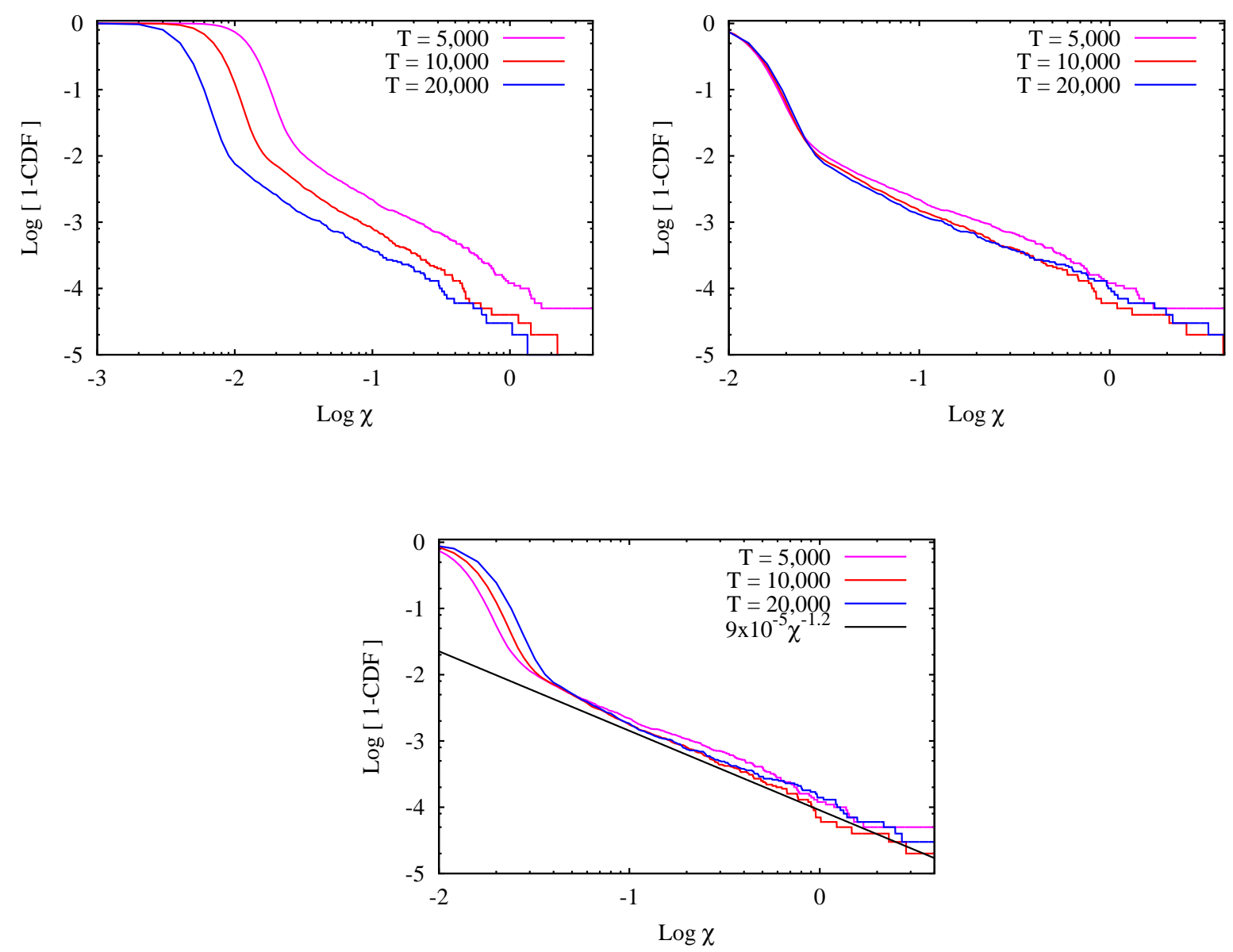

Figure 3.27: Partial collapse for $\chi=L / A$. The results presented in this figure were obtained using Lévy walks with $\alpha=1.2$ for three different time intervals T. Top left: complementary cumulative distribution function of $\chi$. Top right: complementary cumulative distribution function for the rescaled perimeter $\chi / T^{1 / \alpha}$. The Lévy scaling results in a partial collapse. Bottom: complementary cumulative distribution for the rescaled perimeter $\chi / T$. Due to statistical fluctuations in the power law tails of the distributions, we find no clear signatures of a collapse. 
displaying normal diffusion have very similar shapes with a rapidly decaying tail and show no signs of a broad tail. On the other hand, the Lévy walk can be identified by the non-trivial shape of the complementary cumulative distribution comprised of two parts, a rapidly decaying initial part and a separate slowly decaying tail. The crucial identifier or discriminant of the algorithm is the shape of the complementary cumulative distribution curve. An $F(\chi)$ with a simple single-component shape implies that the underlying motion obeys normal diffusion while a two-component shape with an extended "foot" marks the presence of a genuine super-diffusive process.

When the data set is very modest, then even $\chi$ can lead to ambiguous conclusions. An example is shown in figure 3.29. The right panel of the figure (correctly) indicates a broad tail, pointing to super-diffusive motion, however in the left panel neither case can be excluded. As a result, we roughly set a lower bound estimate for $N$ to be around 100 . We have tested other quantities and ratios instead of $\chi$, such as the area $A$ or the ratio $L^{2} / A$, etc., but none of them performed as well as $L / A$.

Finally, the numerical simulations show that the convex hull method is fairly robust to data loss. We emulated the data loss by removing a certain percentage of points from each cloud in the ensemble and then applied the algorithm (see figure 3.30). In figure 3.31 the cumulative distribution of $\chi$ corresponding to the original data is compared to the case where points were removed. The only difference observed is that the reduced dataset produces a cumulative distribution that is shifted towards higher values. The shapes of the curves remain unaffected.

\subsection{Discussion}

Using convex hulls to discriminate between different random processes might be criticised because most of the available data is disregarded. Depending on the properties we aim to determine, in certain cases it is even useful to disregard the details of the system. When dealing with data sets containing recorded animal movement, it is not always easy or even possible to reconstruct the trajectories in order to study them further. It might not be possible to identify the necessary turning and resting points of the animal. Furthermore, the data collected might not be accurate or complete, making it necessary to use interpolation and other techniques in order to make the data set usable. Because of the removal of data, the convex hull method might not be adequate for studying possible correlations in the movement patterns for animals, but we have demonstrated that it is a valid tool for identifying cases where the underlying motion is scale-free. 

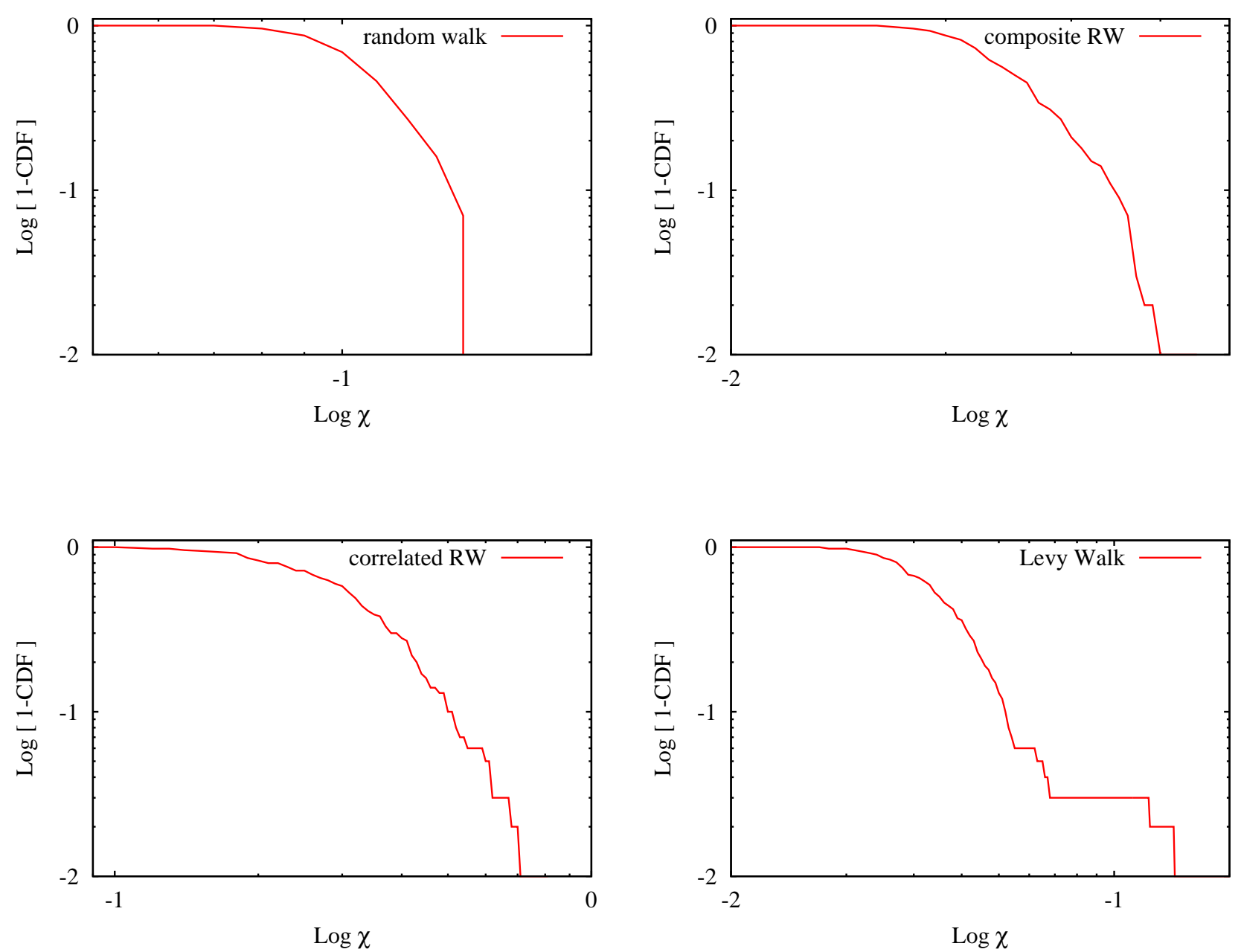

Figure 3.28: Results of the convex hull algorithm - Test for super-diffusion. The algorithm is applied to point-cloud ensembles corresponding to three variants of random walks (representing normal diffusion) and the Lévy walk with $\alpha=1$ (super-diffusion). Each ensemble consists of $N=100$ point-clouds, each recorded in the time interval [0,1000]. Only the bottomright panel shows the tail characteristic of the super-diffusive process. The other three panels show an exponentially decaying curve implying that the underlying motion is normal diffusive, irrespective of the fact whether it is correlated or composite. The correlated random walk consists of direction changes such that $\theta_{i}=\theta_{i-1}+\theta_{\varepsilon}$, where $\theta_{\varepsilon} \in[-\pi / 100, \pi / 100]$. The composite random walk consists of exponentially distributed jump lengths with mean 1 [a.u] that occur with probability 0.9 and exponentially distributed jump lengths of mean 15 [a.u] that occur with probability 0.1. The simple random walk in the top right panel has jump lengths with standard deviation $\sqrt{2}$ [a.u]. 

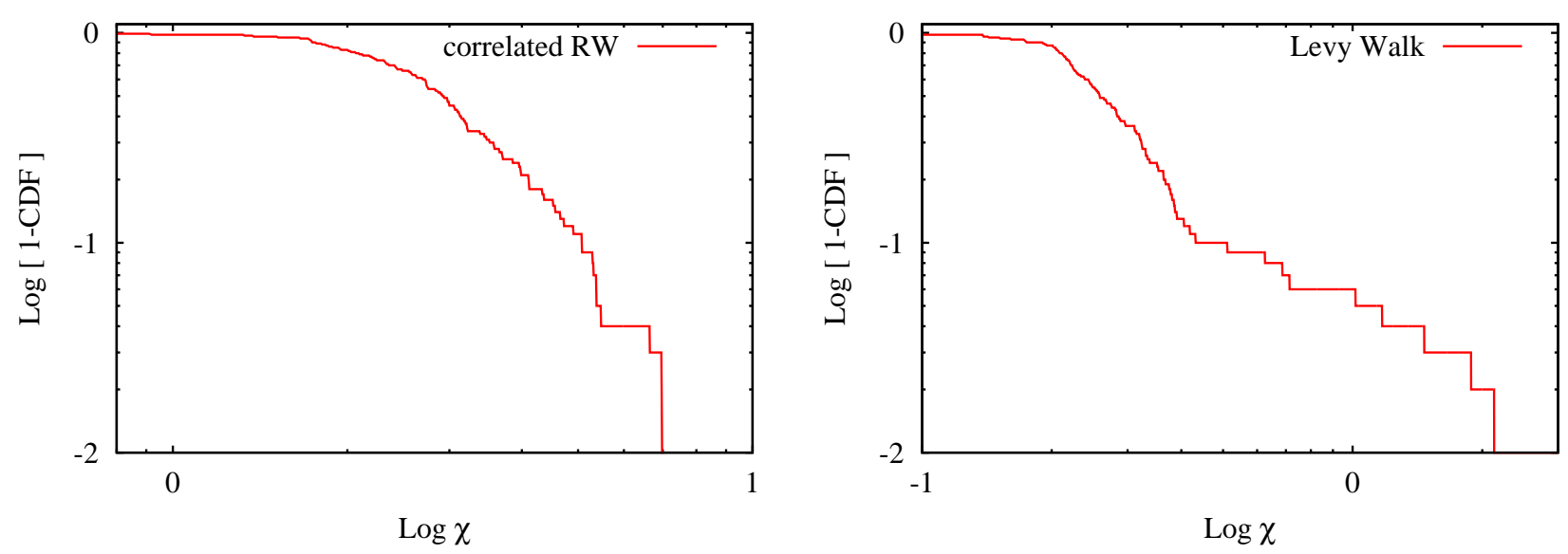

Figure 3.29: The results of the convex hull algorithm applied to a an ensemble of 100 point-clouds. Each point cloud is recorded in the time interval [0,100]. The correlated random walk is identical to the one in figure 3.28 and the Lévy walk has stability parameter $\alpha=1$. In this case, it is very likely for the correlated random walk to be mistaken for a super-diffusive process.

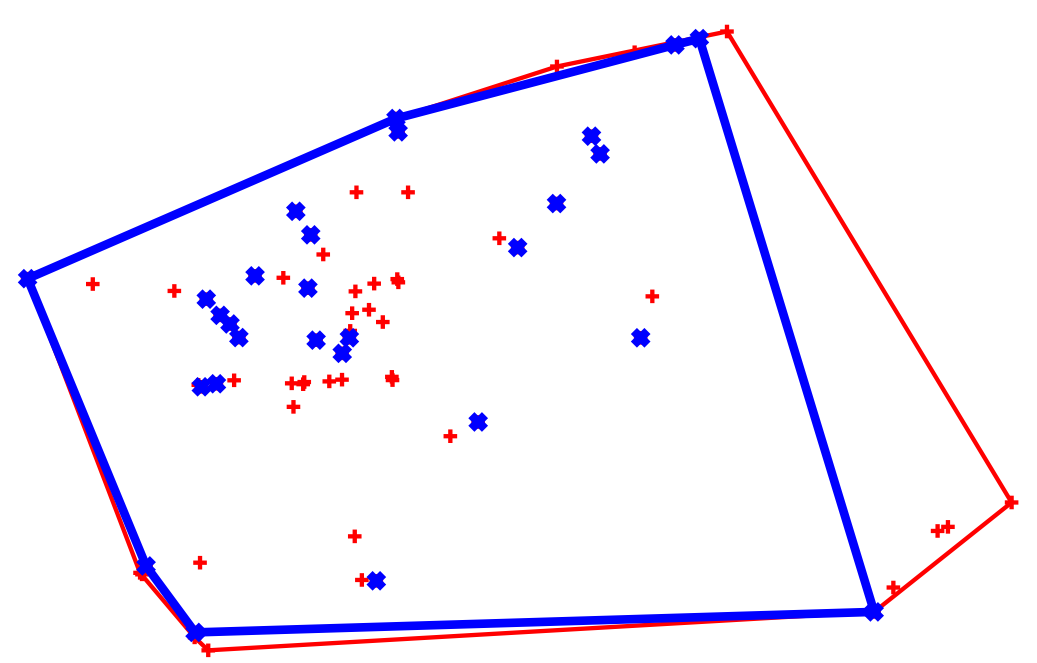

Figure 3.30: Cartoon for the robustness of the method. Reduced data set. The complete point-cloud is represented by the red points. Removing 50\% of the points results in the blue convex hull. 

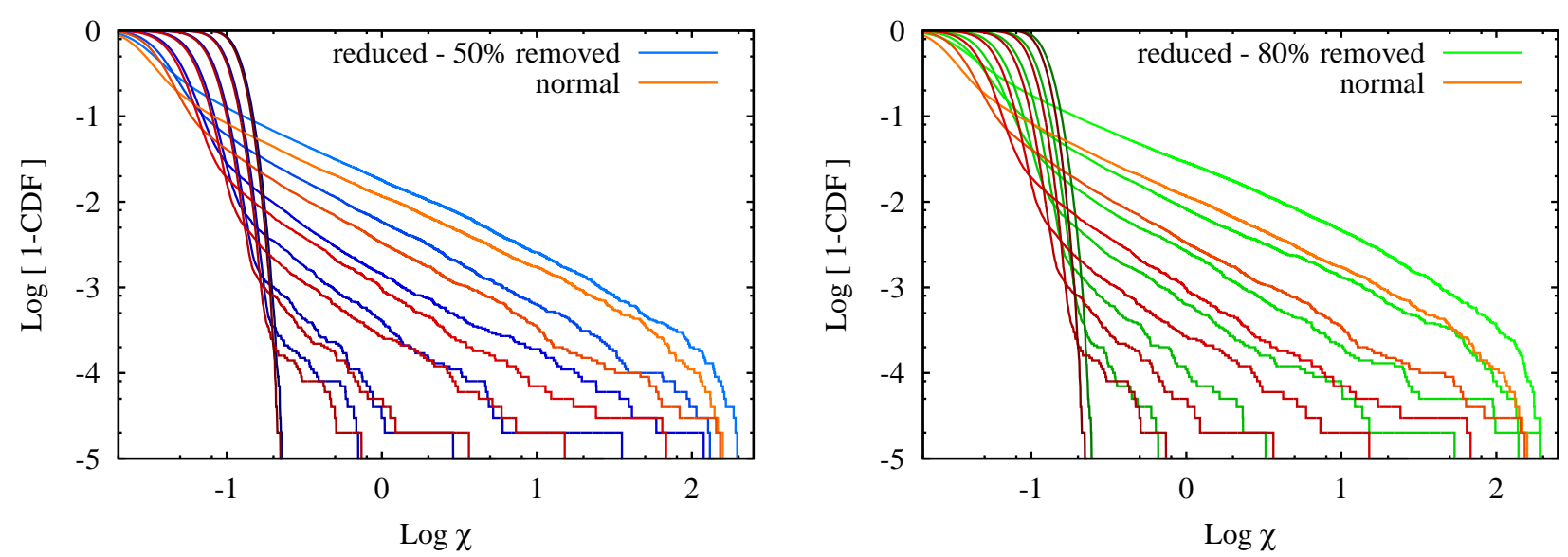

Figure 3.31: Robustness of the method. Reduced datasets - The complementary cumulative distribution function of $\chi=L / A$ corresponding to Lévy walks with different stability parameters $\alpha$. The total walk time is $T=10000$ units with $\alpha$ ranging from 0.8 to 2 (from darker shades to lighter shades) in steps of 0.2. Each ensemble is made of $10^{5}$ point-clouds. The red curves are the result of using all complete point-clouds. By randomly removing 50\% of the points results in the blue curves and removing $80 \%$ of the total points results in the green curves. 


\section{Chapter 4}

\section{Convex Hulls of Continuous Time Random Walks}

In its general form, a continuous time random walk (CTRW) is a random walk where the waiting times between the successive displacements are randomly distributed $[59,60,50]$. Recently the CTRW has been applied to describe anomalous transport in a variety of different complex systems $[61,62,63,64]$. While the bulk of the research focuses on one-dimensional quantities such as the MSD, little is known about two-dimensional properties of CTRWs.

In the present chapter we present an analysis of the convex hull of a CTRW in the plane. To this end we determine analytical expressions for the time-evolution of the average perimeter and area of convex hulls of such processes [65]. It is important to note that, except for the degenerate case of a fixed waiting time, the CTRW is only a Markovian process if the waiting time between the displacements are exponentially distributed. Here we focus on the case of a heavy-tailed waiting time distribution with an infinite mean. Thus for the first time we provide analytical calculations for the convex hull of a class of genuinely non-Markovian processes.

The continuous time random walk can be considered as an example of an intermittent search model. Our results can be applied to model the home range of foraging animals that perform a saltatory, intermittent search strategy for their prey. Such an intermittent locomotion can be advantageous for a variety of reasons. The pausing times between displacements help animals recover from fatigue, search more accurately for prey or evade predators more efficiently [66]. An example are rattlesnakes, which remain in the same position for extended periods of time waiting to ambush a potential prey [67].

Furthermore, there is growing evidence that human activity is drastically changing the foraging habits of animals, forcing them to adopt sub-diffusive search strategies. An example for such an induced change of a behavioural pattern are the effects of human fishing on seabirds such as the Balearic shearwater and the Cory's shearwater in the Mediterranean [68]. Due to the presence of trawlers these birds start showing strong site fidelity to certain foraging areas, thus making the overall foraging process sub-diffusive. Since the CTRW is a model of diffusion with trapping events, our considerations are also of interest in the context of ground water 
pollution in porous layers where the diffusion is known to be anomalous [64].

\subsection{Subordinated Brownian Motion}

A simple random walk in one dimension is characterised by a sequence of jumps of random length $\lambda$. For the sake of simplicity, we assume that the jump lengths are independent identically distributed random variables sampled from a symmetric distribution function $\varphi(\lambda)$ with finite variance. We shall use $p_{n}(x)$ to denote the probability of finding a random walker in position $x=\lambda_{1}+\lambda_{2}+\ldots+\lambda_{n}$ exactly after $n$ jumps.

As mentioned earlier, the CTRW is a generalization of the random walk whereby random waiting times $\{\tau\}$ are assumed to take place between the random jumps. In order to preserve causality, the $\{\tau\}$ have to be greater than zero. Furthermore we assume the waiting times to be independent identically distributed positive random variables sampled from a distribution $\psi(\tau)$ that is independent of $\varphi(\lambda)$. In that case, the probability of finding the random walker at $x$ after a time $t$ is given by $p(x, t)=\sum_{n=0}^{\infty} K_{n}(t) \cdot p_{n}(x)$. Here $K_{n}(t)$ denotes the probability that exactly $n$ jumps occurred up to the time $t$ which reads in the Laplace domain $\tilde{K}_{n}(u)=\tilde{\psi}^{n}(u) \cdot \tilde{\Psi}(u)$, where $\Psi(\tau)$ is the survival function denoting the probability that no jump occurs upto time $\tau$ $[60,50]$. Here and in the following $\tilde{f}(u)=\mathcal{L}\{f(t)\}$ denotes the Laplace transform.

The function $K_{n}(t)$ can be considered as the kernel of a transform that maps a probability density from the domain of an operational time $n$ to that of the physical time $t$. In the mathematical literature one refers to the random walk $x(n)$ as the parent process and the CTRW as the process $x(t)=x[n(t)]$ subordinated to $x(n)$. Figure 4.1 shows a graphical representation of subordination.

In this paper we consider the scaling limit of the CTRW which is called subordinated Brownian motion. Since Brownian motion is equivalent to the diffusive limit of a random walk, the series representation of the CTRW shown above has to be substituted, using proper scaling relations, by an integral form [69]. Here we use an intuitive, albeit not so formal, approach introduced by Fogedby [70]. He considered the scaling limit of a CTRW via a set of coupled Langevin equations of the form

$$
\frac{\mathrm{d} x}{\mathrm{~d} s}=\xi(s), \quad \frac{\mathrm{d} t}{\mathrm{~d} s}=\eta(s),
$$

where $\xi(s)$ and $\eta(s)$ are random noise sources independent of each other and $s$ the continuous equivalent of the operational time $n$ which is sometimes referred to as internal time. Under these circumstances, the equation on the left in (4.1) is the parent process and the one on the right relates the physical time to the operational time. Analogous to the discrete case, the values of $\eta$ have to be strictly positive in order to insure causality. Furthermore, the continuous equivalent of the kernel function $K_{n}(t)$, defined as $K(s, t)$, is the probability density associated to $s(t)$, the inverse of the stochastic process $t(s)$. For this reason, the existence of $s(t)$ is essential, in which case $t(s)$ must be a non-decreasing right-continuous function. 


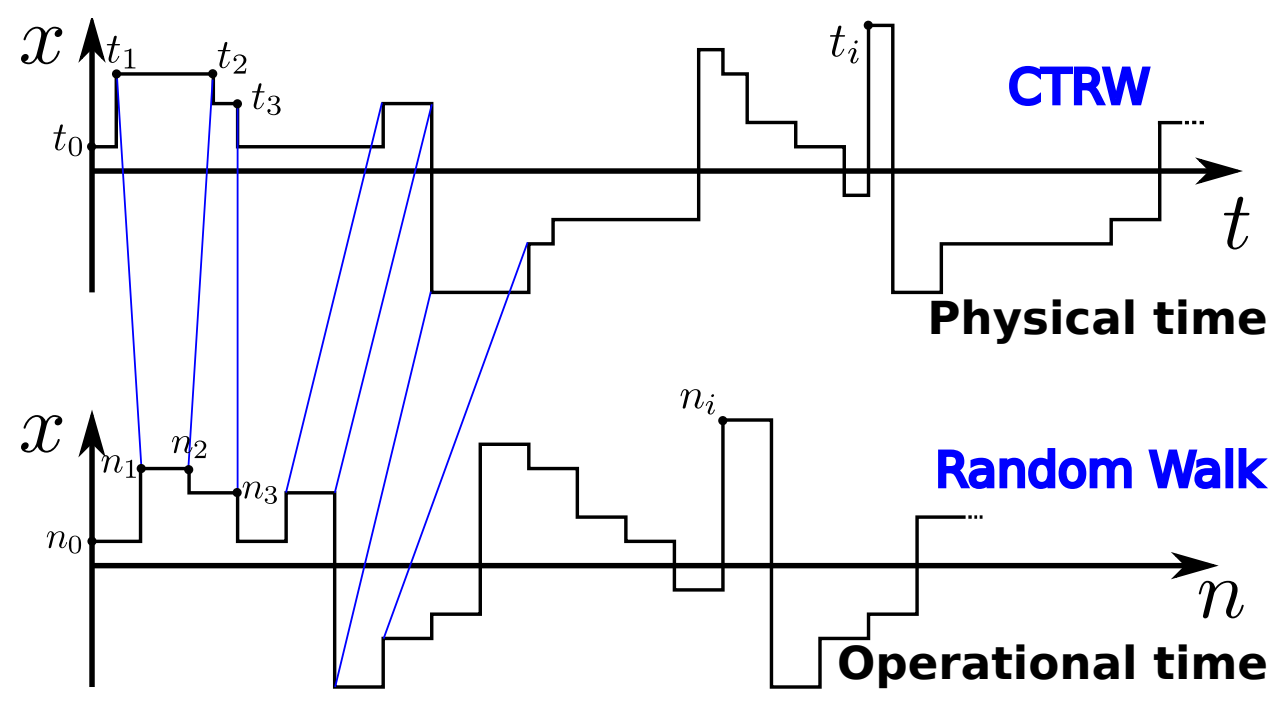

Figure 4.1: A schematic representation of subordination. The figure shows how a continuous time random walk can be obtained from a normal random walk. The discrete time intervals of the normal random walk are replaced by a random variable sampled from an inverse power law.

It can be shown that the set of coupled Langevin equations (4.1) leads to a time-fractional diffusion equation if the random variable $\eta$ is sampled from a heavy-tailed probability density such as the one-sided $\alpha$-stable distribution and if $\xi(s)$ is assumed to be white noise [70]. In other words, if we choose a waiting time probability density with asymptotic behaviour $\psi(\eta) \sim$ $\alpha b_{\alpha} \eta^{-1-\alpha} / \Gamma(1-\alpha)$ where $0<\alpha<1$ and assume $x(s)$ to be a Wiener process, then the resulting stochastic process $x(t)$ in the physical time domain that emerges from (4.1) is non-Markovian and sub-diffusive. Note that $b_{\alpha}$ is a constant with units $\left[b_{\alpha}\right]=\left[T_{t}^{\alpha}\right]$ in physical time $t$.

In the Laplace domain the waiting time distribution has the asymptotic behaviour $\tilde{\psi}(u) \simeq$ $1-b_{\alpha} u^{\alpha}$ so that in the scaling limit one obtains $K_{\alpha}(s, u)=c_{\alpha} u^{\alpha-1} \exp \left(-s c_{\alpha} u^{\alpha}\right)$, where $c_{\alpha}=b_{\alpha} \cdot r$ with the constant $r$ being the number of steps per unit operational time [70]. We therefore have $\left[c_{\alpha}\right]=\left[T_{t}^{\alpha}\right] /\left[T_{s}\right]$. Laplace inversion then yields $[69,71]$

$$
K_{\alpha}(s, t)=\frac{1}{c_{\alpha}^{1 / \alpha}} \frac{t}{\alpha s^{1+1 / \alpha}} L_{\alpha}\left(\frac{t}{c_{\alpha}^{1 / \alpha} s^{1 / \alpha}}\right),
$$

where $L_{\alpha}(t)$ is the one-sided Lévy-stable distribution with stability parameter $0<\alpha<1$ whose Laplace transform is given by $\tilde{L}_{\alpha}(u)=\exp \left(-u^{\alpha}\right)[72]$.

Combining the distributions corresponding to the two processes $x(s)$ and $s(t)$, i.e. $p(x, s)$ and $K_{\alpha}(s, t)$ respectively, we can eliminate the internal time to finally obtain the propagator for the subordinated process:

$$
p_{\alpha}(x, t)=\int_{0}^{\infty} \mathrm{d} s K_{\alpha}(s, t) \cdot p(x, s) .
$$


This result is central to subordination theory and will be used frequently in the following. Note that $p_{\alpha}(x, t)$ can be considered as the solution of a non-Markovian diffusion equation, connected to its standard Markovian counterpart, $p(x, s)$, through (4.3). This equation is valid in general as long as the two functions in the integrand remain non-negative [73]. In other words, as formulated by Metzler and Klafter, the transformation (4.3) guarantees the existence and positivity of $p_{\alpha}(x, t)$ if (and only if) the Brownian counterpart, $p(x, s)$, is a proper probability density function [74].

\subsection{Geometric properties}

Since the focus of this letter is on the properties of convex hulls of CTRW processes, we shall shortly summarize some important results of the theory of random hulls which are especially well suited to treat correlated stochastic processes. It is known that the perimeter $L(T)$ and area $A(T)$ of the convex hull of a single path can be determined using the Cauchy functionals $[13,37]:$

$$
L(T)=\int_{0}^{2 \pi} \mathrm{d} \theta M(\theta)
$$

and

$$
A(T)=\frac{1}{2} \int_{0}^{2 \pi} \mathrm{d} \theta\left[M^{2}(\theta)-(\mathrm{d} M(\theta) / \mathrm{d} \theta)^{2}\right]
$$

where $M(\theta)$, which is referred to as the support function, is the maximum extent of the projection of the given stochastic path in the direction of the angle $\theta \in[0,2 \pi]$. For any planar stochastic path $(x(t), y(t))$ in continuous time $t \in[0, T]$ the support function has the form

$$
M(\theta)=\max _{t \in[0, T]}\{x(t) \cos \theta+y(t) \sin \theta\}
$$

Figure 4.2 gives a geometric interpretation of the support function and its derivative. A concise derivation of these results is provided in [13].

We shall now proceed to calculate the properties of the random convex hull enclosing the stochastic path $\mathbf{r}(t)=(x(t), y(t))$ traced by a CTRW in the $x y$ plane in the time interval $0<t<T$. In order to calculate the average perimeter and area of such a process, we have to determine the support function associated with it. As shown in (4.6), the support function depends on the angle $\theta$ with respect to the $x$-axis and an arbitrarily chosen origin, for which we will use the starting point of the stochastic process.

With (4.6) in mind, we introduce $z_{\theta}(t)=x(t) \cos \theta+y(t) \sin \theta$ so that the support function can be written as $M(\theta)=\max _{t \in[0, T]}\left\{z_{\theta}(t)\right\}$. Furthermore, let us denote $h_{\theta}(t)$ to be the derivative of $z_{\theta}(t)$ with respect to $\theta$. At some point within the time interval $[0, T]$ the planar CTRW will reach its maximum excursion in the direction $\theta$. Let us denote this time with $\tau_{m}$ 


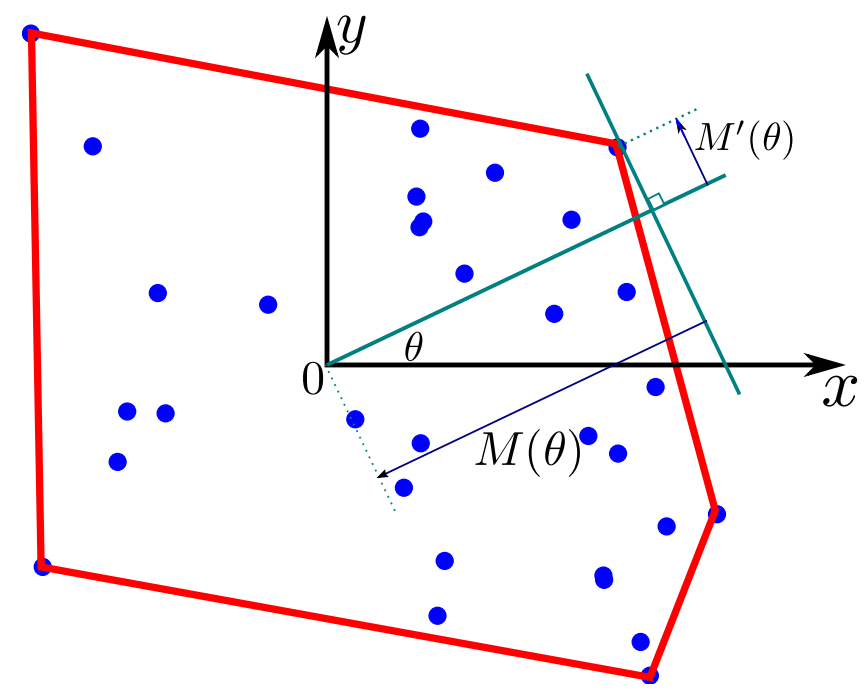

Figure 4.2: Support function and the convex hull of a set of randomly distributed points. $M(\theta)$ is the support function and $M^{\prime}(\theta)$ its derivative. The support function is the maximum extent of the set of random points along the direction of $\theta$ from the origin $O$.

and use $\rho_{\alpha}\left(\tau_{m}, T\right)$ for the corresponding probability density function. The support function and its derivative can then be written as

$$
\begin{aligned}
M(\theta) & =z_{\theta}\left(\tau_{m}\right)=x\left(\tau_{m}\right) \cos \theta+y\left(\tau_{m}\right) \sin \theta \\
M^{\prime}(\theta) & =h_{\theta}\left(\tau_{m}\right)=-x\left(\tau_{m}\right) \sin \theta+y\left(\tau_{m}\right) \cos \theta .
\end{aligned}
$$

The quantity $M^{\prime}(\theta)$ gives the value of the projection of the planar CTRW onto the direction perpendicular to $\theta$ attained at time $\tau_{m}$. In the particular case where $\theta=0$ we have that $z_{0}(t)=x(t)$ and $h_{0}(t)=y(t)$ so that the support function reduces to $M(0)=z_{0}\left(\tau_{m}\right)=x_{m}$ while its derivative is given by $M^{\prime}(0)=h_{0}\left(\tau_{m}\right)=y_{m}$.

Calculating the distributions of the hull perimeter $L(T)$ and area $A(T)$ is very difficult in the Brownian case, let alone for CTRWs. Therefore, in this paper we settle with the task of calculating the average values of these quantities. Since we neglect any external biases, the process under consideration is isotropic in space. Thus, we can take $\theta$ to be zero without loss of generality and write down the expressions for the average perimeter and area respectively as

$$
\langle L(T)\rangle=2 \pi\left\langle x_{m}\right\rangle
$$

and

$$
\langle A(T)\rangle=\pi\left(\left\langle x_{m}^{2}\right\rangle-\left\langle y_{m}^{2}\right\rangle\right) .
$$

where $\langle\cdot\rangle$ denotes an ensemble average. 


\subsubsection{Average perimeter}

According to (4.9), the average perimeter of the convex hull of a planar CTRW can be determined using the average maximum excursion of the one-dimensional stochastic process $z_{0}(t)$ in the interval $[0, T]$. Hence we need to calculate the density function $f_{\alpha}\left(x_{m}, T\right)$ for the maximum positive-valued excursion of the process $z_{0}(t)$. In the case of Brownian motion, it is well known that the probability density of the maximum positive excursion from the origin achieved in the time interval $[0, S]$ is given by $[75]$

$$
f\left(x_{m}, S\right)=(\pi D S)^{-1 / 2} \exp \left(-x_{m}^{2} /(4 D S)\right),
$$

where $D=r\left\langle\lambda^{2}\right\rangle / 2$ is the diffusion constant of the underlying Brownian motion with units $[D]=$ $\left[L^{2}\right] /\left[T_{s}\right]$. This result, together with the subordination concept can be employed to calculate the maximum excursion density $f_{\alpha}\left(x_{m}, T\right)$ of a CTRW in the physical time $T$. Substituting $f\left(x_{m}, S\right)$ into (4.3) one gets

$$
f_{\alpha}\left(x_{m}, T\right)=\int_{0}^{\infty} \mathrm{d} S K_{\alpha}(S, T) \cdot f\left(x_{m}, S\right) .
$$

Laplace transforming (4.12) yields $\tilde{f}_{\nu}(z, u)=\left(c_{2 \nu} D\right)^{-1 / 2} \tilde{K}_{\nu}(z, u)$, where $\nu=\alpha / 2$ and $z=$ $\left|x_{m}\right| / \sqrt{c_{2 \nu} D}$. Back transformation then provides the distribution of the maximum

$$
f_{\nu}(z, T)=\frac{\left(c_{2 \nu} D\right)^{-1 / 2}}{\nu c_{2 \nu}^{1 / \nu}} \frac{T}{z^{1+1 / \nu}} \mathcal{L}_{\nu}\left(\frac{T}{\left(c_{2 \nu} z\right)^{1 / \nu}}\right),
$$

which, for $D=1$, confirms the result obtained by Schehr et al. with the real space renormalisation group method [76]. This result was also obtained by Carmi et al. using functionals of sub-diffusive CTRWs [77].

Having the analytical expression for $f_{\alpha}\left(x_{m}, T\right)$, the first moment $\left\langle x_{m}(T ; \alpha)\right\rangle$ can be calculated (see 3.6 of [50]) and we obtain for the average perimeter of a planar CTRW:

$$
\langle L(T)\rangle=\frac{2 \pi \sqrt{D_{\alpha}}}{\Gamma(1+\alpha / 2)} T^{\alpha / 2},
$$

where $D_{\alpha}=D / c_{\alpha}$ is the generalised diffusion constant with units $\left[D_{\alpha}\right]=\left[L^{2}\right] /\left[T_{t}^{\alpha}\right]$.

\subsubsection{Average Area}

The determination of the average area of the convex hull of a planar CTRW is slightly more involved. From (4.10) it is apparent that we need to calculate the moments $\left\langle x_{m}^{2}\right\rangle$ and $\left\langle y_{m}^{2}\right\rangle$. While $\left\langle x_{m}^{2}\right\rangle$ can be extracted directly from the probability density function (4.13), giving

$$
\left\langle x_{m}^{2}(T ; \alpha)\right\rangle=\int_{0}^{\infty} \mathrm{d} x_{m} x_{m}^{2} \cdot f_{\alpha}\left(x_{m}, T\right)=\frac{2 D_{\alpha}}{\Gamma(1+\alpha)} T^{\alpha},
$$

the calculation of $\left\langle y_{m}^{2}\right\rangle$ is not so straightforward. In principle we need to know $g_{\alpha}\left(y_{m}, T\right)$ i.e. the probability density of the value of $y\left(\tau_{m}\right)$ attained at the instance when the process $x\left(\tau_{m}\right)$ 
reaches its maximum excursion in the positive direction in the time interval $[0, T]$. However, the difficulties of calculating $g_{\alpha}\left(y_{m}, T\right)$ arise due to the fact that the two one-dimensional projections $x(t)$ and $y(t)$ of the two-dimensional CTRW are not independent. In contrast to the Markovian case, when a planar CTRW is projected onto the $x$ and $y$ direction, there always remains a correlation in the time of the "jumps". The two one-dimensional projections always change direction simultaneously, no matter how the decomposition is done.

The way around this problem is again to use subordination. Therefore we note that the parent process can be decomposed into two independent one-dimensional Brownian motions. The trick is then to subordinate these two processes to the same subordinator, i.e. we have to consider the Langevin system

$$
\dot{x}(s)=\xi_{x}(s), \quad \dot{y}(s)=\xi_{y}(s), \quad \dot{t}(s)=\eta(s),
$$

where $\xi_{x}(s)$ and $\xi_{y}(s)$ are two independent realizations of the same white noise source and $\eta(s)$ is chosen as before (see figure 4.3).

Discrete time, $\mathrm{n}$

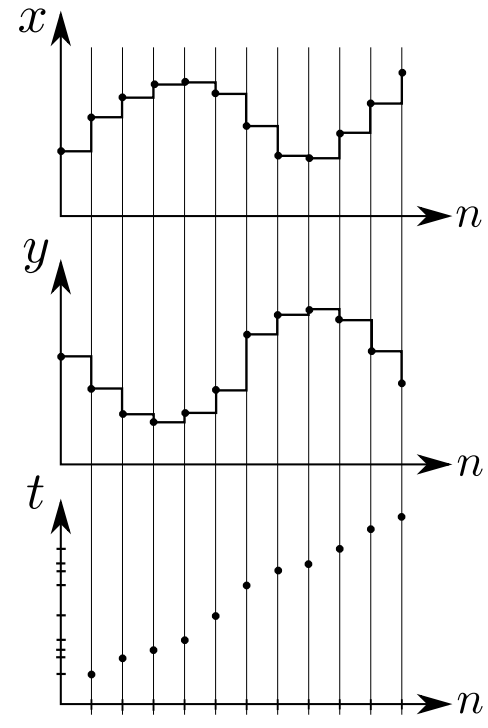

Continuous time, $\mathrm{s}$

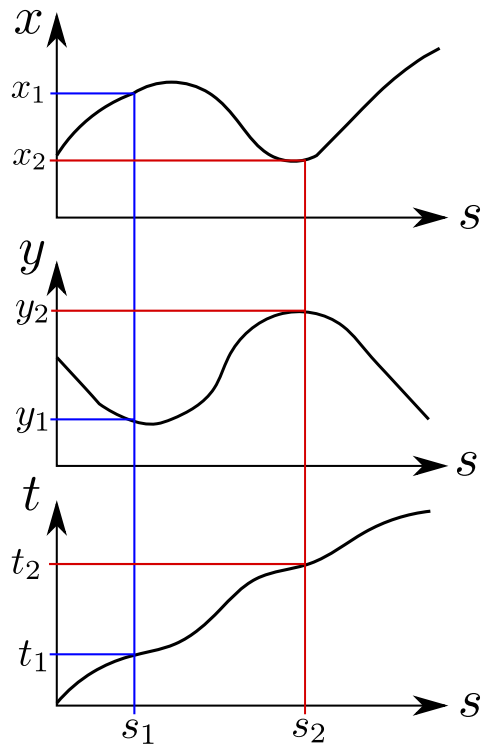

Equation

$$
\dot{x}(s)=\xi_{x}(s)
$$$$
\dot{y}(s)=\xi_{y}(s)
$$$$
\dot{t}(s)=\tau(s)
$$

Figure 4.3: Schematic representation of subordination in continuous time. A cartoon of a single realisation of the Brownian motion and its discrete counterpart is shown.

On their own, the first two equations in (4.16) constitute a planar Brownian motion whose two components $x(s)$ and $y(s)$ are independent and are governed by the same propagator $p(\cdot, s)$. Therefore, in operational time it is legitimate to express the probability density governing the random variable $y_{m}$ as

$$
g\left(y_{m}, S\right)=\int_{0}^{S} \mathrm{~d} \sigma_{m} p\left(y_{m}, \sigma_{m}\right) \cdot \rho\left(\sigma_{m}, S\right),
$$


where $\sigma_{m} \in[0, S]$ is the time when the process $x(s)$ reaches its maximum whose probability density $\rho\left(\sigma_{m}, S\right)=\left[\sigma_{m}\left(S-\sigma_{m}\right)\right]^{-1 / 2} / \pi$ is given by the famous arcsine law [75], and $p(y, s)$ is the propagator associated to $y(s)$. It is important to observe that such a decomposition of $g$ is not possible for the CTRW, due to the correlations between $x(t)$ and $y(t)$ in the physical time $t$. Since $y(s)$ is the trajectory of a Brownian motion and therefore $p\left(y_{m}, \sigma_{m}\right)=\left(4 \pi D \sigma_{m}\right)^{-1 / 2} \exp \left(-y_{m}^{2} /\left(4 D \sigma_{m}\right)\right)$, we can determine the integral in (4.17) in terms of the zeroth order modified Bessel function of the second kind, $\mathcal{K}_{0}(\cdot)$ :

$$
g\left(y_{m}, S\right)=\frac{\mathcal{K}_{0}\left(\frac{y_{m}^{2}}{8 D S}\right)}{2 \pi^{3 / 2} \sqrt{D S}} \exp \left(-\frac{y_{m}^{2}}{8 D S}\right) .
$$

By linking the probability density functions governing $y_{m}$ in the two time domains we are now able to determine $g_{\alpha}\left(y_{m}, T\right)$. Substituting $g\left(y_{m}, S\right)$ into (4.3) one obtains

$$
g_{\alpha}\left(y_{m}, T\right)=\int_{0}^{\infty} \mathrm{d} S K_{\alpha}(S, T) \cdot g\left(y_{m}, S\right) .
$$

Having this equation in mind, the formal expression for the second moment of the random variable $y_{m}$ in the physical time domain is given by

$$
\left\langle y_{m}^{2}(T ; \alpha)\right\rangle=\int_{-\infty}^{\infty} \mathrm{d} y_{m} y_{m}^{2} \int_{0}^{\infty} \mathrm{d} S K_{\alpha}(S, T) \cdot g\left(y_{m}, S\right)
$$

The second moment can be evaluated by first applying the Laplace transform to (4.20) and then integrating over $y_{m}$ and finally anti-Laplace transforming the result into the $T$-domain. The Laplace transform of (4.19) yields

$$
\begin{aligned}
\tilde{g}_{\alpha}\left(y_{m}, u\right) & =\int_{0}^{\infty} d S \frac{\mathcal{K}_{0}\left(\frac{y_{m}^{2}}{8 D S}\right)}{2 \pi^{3 / 2} \sqrt{D S}} \exp \left(-\frac{y_{m}^{2}}{8 D S}\right) \cdot c_{\alpha} u^{\alpha-1} \exp \left(-S c_{\alpha} u^{\alpha}\right) \\
& =b \int_{0}^{\infty} d S \mathcal{K}_{0}\left(\frac{y_{m}^{2}}{8 D S}\right) \exp \left(-\frac{y_{m}^{2}}{8 D S}-S c_{\alpha} u^{\alpha}-\ln (S) / 2\right),
\end{aligned}
$$

where $b=\frac{1}{2 \pi^{3 / 2}} \frac{c_{\alpha} u^{\alpha-1}}{\sqrt{D}}$. A further simplification is obtained by noticing that the Bessel function can be expressed as the integral,

$$
\mathcal{K}_{0}(x)=\int_{0}^{\infty} d t \exp (-x \cosh (t))
$$

Substituting (4.22) into (4.21) gives

$$
\begin{aligned}
\tilde{g}_{\alpha}\left(y_{m}, u\right) & =b \int_{0}^{\infty} d t \int_{0}^{\infty} d S \exp \left(-\frac{y_{m}^{2}}{8 D S}(1+\cosh (t))-S c_{\alpha} u^{\alpha}-\ln (S) / 2\right) \\
& =b \int_{0}^{\infty} d t \sqrt{\frac{\pi}{c_{\alpha} u^{\alpha}}} \exp \left(-\sqrt{\frac{c_{\alpha} u^{\alpha}}{D}}\left|y_{m}\right| \cosh (t / 2)\right) \\
& =2 b \sqrt{\frac{\pi}{c_{\alpha} u^{\alpha}}} \mathcal{K}_{0}\left(\sqrt{\frac{c_{\alpha} u^{\alpha}}{D}}\left|y_{m}\right|\right) .
\end{aligned}
$$


Therefore, with the help of any standard text on integrals of special functions, we have

$$
\left\langle y_{m}^{2}(u ; \alpha)\right\rangle=\frac{1}{u \pi} \sqrt{\frac{c_{\alpha} u^{\alpha}}{D}} \int_{-\infty}^{\infty} \mathrm{d} y_{m} y_{m}^{2} \mathcal{K}_{0}\left(\sqrt{\frac{c_{\alpha} u^{\alpha}}{D}}\left|y_{m}\right|\right)=D_{\alpha} u^{-1-\alpha} .
$$

Applying the Tauberian theorem on Laplace transforms [50], we obtain

$$
\left\langle y_{m}^{2}(T ; \alpha)\right\rangle=\frac{D_{\alpha}}{\Gamma(1+\alpha)} T^{\alpha}
$$

Finally, by substituting the last two results into (4.10), one obtains as the second central result of this letter for the average area of the convex hull of a CTRW

$$
\langle A(T)\rangle=\frac{\pi D_{\alpha}}{\Gamma(1+\alpha)} T^{\alpha} .
$$

For $\alpha=1$, both, the perimeter (4.14) and the area (4.26) reduce to the well known result for the Brownian case [13].

\subsubsection{Discussion}

One might argue that the results in (4.14) and (4.26) could have been obtained by applying the subordination transformation directly to the mean perimeter and area relative to the Brownian case. However, when dealing with the subordination method, the only way to be sure of obtaining meaningful results is to work with probability densities [73, 74].

To verify our analytical results (4.14) and (4.26) we have performed numerical simulations. To this end an ensemble of two-dimensional CTRWs was created and the convex hulls around them were constructed using the Graham scan [39] (see appendix for details). Figure 4.4 shows a perfect agreement of the analytical results with the simulations.

\subsection{Subordinated Lévy flights}

So far we only considered the case where the distribution of the displacements has a finite variance. Some of the results, however, can be generalised to the case of Lévy Flights which are characterised by a heavy-tailed jump distribution of the form $\varphi(\lambda) \sim B_{\mu} /|\lambda|^{1+\mu}$, where $B_{\mu}$ is a constant. In particular, we will consider jump distributions whose characteristic function has the form $\hat{\varphi}(k)=\exp \left(-a_{\mu}|k|^{\mu}\right) \simeq 1-a_{\mu}|k|^{\mu}$. For $1<\mu<2$ this distribution has a finite mean but a diverging variance and the Lévy Flight exhibits super-diffusive behaviour. On the other hand, $\mu=2$ recovers the Gaussian distribution with standard deviation $\sigma=\sqrt{2 a_{2}}$.

According to (4.4), in order to calculate the mean perimeter of a subordinated Lévy Flight we need to know the mean value of the maximum excursion of the corresponding one-dimensional process which is given by

$$
\left\langle x_{m}(T ; \alpha, \mu)\right\rangle=\int_{-\infty}^{\infty} \mathrm{d} x_{m} x_{m} \int_{0}^{\infty} \mathrm{d} S K_{\alpha}(S, T) \cdot f_{\mu}\left(x_{m}, S\right),
$$



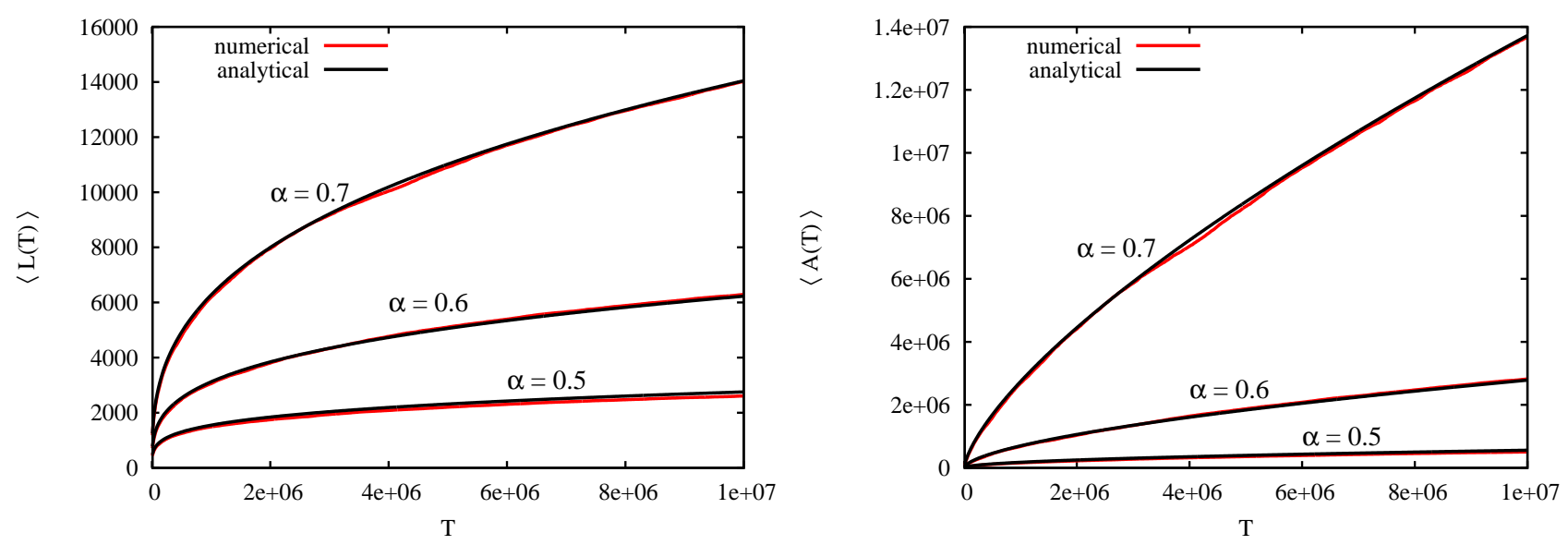

Figure 4.4: Time evolution of the average perimeter (left panel) and area (right panel) of the convex hull of a CTRW for different values of $\boldsymbol{\alpha}$. We observe perfect agreement of the analytical results (4.14) and (4.26) and the numerical simulations.

where $f_{\mu}\left(x_{m}, S\right)$ is the probability distribution of the maximum excursion of a Lévy Flight after time $S$. At first glance this imposes a problem since, to the best of our knowledge, the exact expression for $f_{\mu}\left(x_{m}, S\right)$ is not known. It is difficult to calculate analytically the pdf of the maximum excursions of Lévy flights mainly because the method of images does not apply due to the presence of non local jumps. Nevertheless, the leading order behaviour of the mean maximum $\left\langle x_{m}(S ; \mu)\right\rangle$ of a Lévy Flight after $S$ steps can be alternatively obtained by employing an asymptotic expansion of the Pollaczek-Spitzer formula as was shown by Comtet and Majumdar in [78]. They obtained

$$
\left\langle x_{m}(S ; \mu)\right\rangle=\left(D_{\mu}\right)^{1 / \mu} \frac{\mu}{\pi} \Gamma(1-1 / \mu) S^{1 / \mu}+\mathcal{O}(1)
$$

where $D_{\mu}=a_{\mu} \cdot r$ is the generalised diffusion coefficient of Lévy flights in operational time with units $\left[D_{\mu}\right]=\left[L^{\mu}\right] /\left[T_{s}\right]$ and $a_{\mu}^{1 / \mu}$ the scale parameter of the jump distribution. Since averaging is a linear operation we can exchange the orders of integration in (4.27) yielding

$$
\left\langle x_{m}(T ; \alpha, \mu)\right\rangle=\int_{0}^{\infty} \mathrm{d} S K_{\alpha}(S, T) \cdot\left\langle x_{m}(S ; \mu)\right\rangle .
$$

Observe that a similar expression has recently been obtained in [79]. Inserting (4.28) into (4.29) and applying (4.4) we finally obtain for the mean perimeter of a subordinated Lévy Flight

$$
\langle L(T)\rangle=2\left(\frac{D_{\mu}}{c_{\alpha}}\right)^{1 / \mu} \frac{\Gamma(1-1 / \mu) \Gamma(1 / \mu)}{\Gamma(1+\alpha / \mu)} T^{\alpha / \mu} .
$$

In this case we also find excellent agreement between the analytical results and the simulations (see figure 4.5). Moreover, the result in (4.30) reduces to (4.14) for $\mu=2$ as it should. Note that in the Gaussian case $D=\sigma^{2} r / 2$ and that $\sigma=\sqrt{2 a_{2}}$ when $\mu=2$. 


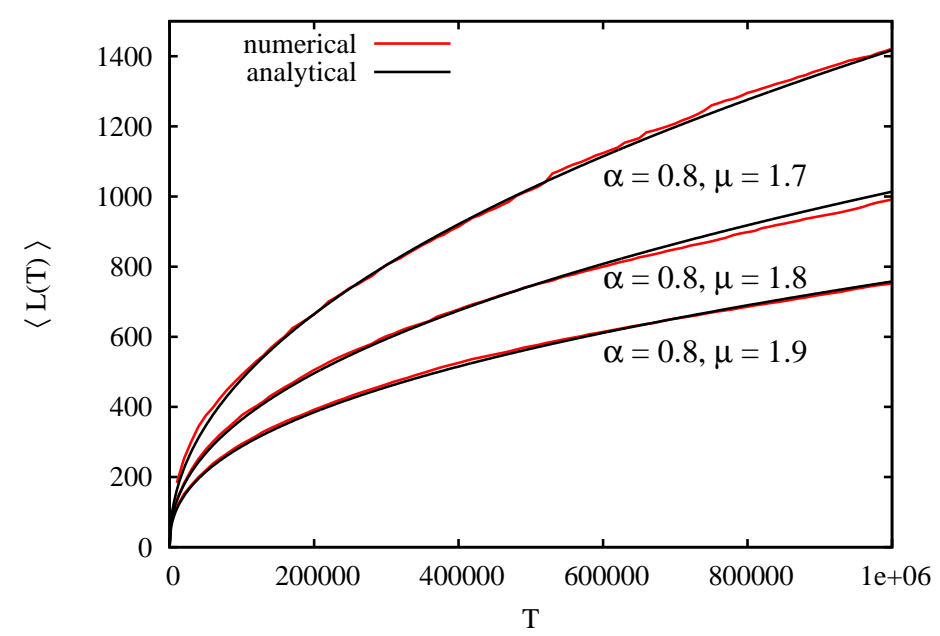

Figure 4.5: Time evolution of the average perimeter of the convex hull of a subordinated Lévy flight. The three red curves are the numerical results obtained using Lévy flights with the same time stability parameter $\alpha=0.6$ and three different values of the spacial stability parameter $\mu$. The simulations agree well with the analytical solution (4.30) (black lines).

The average area of the convex hull of a subordinated Lévy Flight is divergent since already the mean square displacement of a Lévy Flight diverges, i.e. $\left\langle x^{2}(t)\right\rangle=\infty$ for all times.

It is well-known that a Lévy Flight can also be obtained by the subordination of a normal random walk [80]. Intuitively, this means that we can obtain a Lévy flight by irregularly sampling trajectories of Brownian motion (see figure 4.6). Specifically, the sampling is done randomly according to an inverse power law probability distribution with exponent $1<\gamma<2$.

Analogous to the case of the continuous time random walk (see 4.2), a kernel of a transform $K(s, t)$, which maps probability densities from the domain of operational time $s$ to that of physical time $t$, can be derived also for Lévy flights. It is given by [80]

$$
K_{\beta}(s, t)=\frac{1}{t^{1 / \beta}} L_{\beta}\left(\frac{s}{t^{1 / \beta}}\right),
$$

where $L_{\beta}$ is again the one-sided Lévy-stable distribution with stability parameter $0<\beta<1$. The propagator $p_{\alpha}(x, t)$ of symmetric Lévy flight with $1<\alpha<2$ can therefore be expressed in the form of a transform [81]:

$$
p_{\alpha}(x, t)=\frac{1}{t^{1 / \alpha}} L_{s y m}\left(\frac{x}{t^{1 / \alpha}} ; \alpha\right)=\int_{0}^{\infty} \mathrm{d} s \frac{1}{\sqrt{4 \pi s}} \exp \left(-\frac{x^{2}}{4 s}\right) \cdot K_{\alpha / 2}(s, t),
$$

where $L_{\text {sym }}(x ; \alpha)$ is the symmetric Lévy stable distribution.

The propagator $p(x, s)$ of Brownian motion differs only by a factor of one half from the corresponding maximum excursion distribution $f\left(x_{m}, S\right)$ (see (4.11)). Therefore, using the 

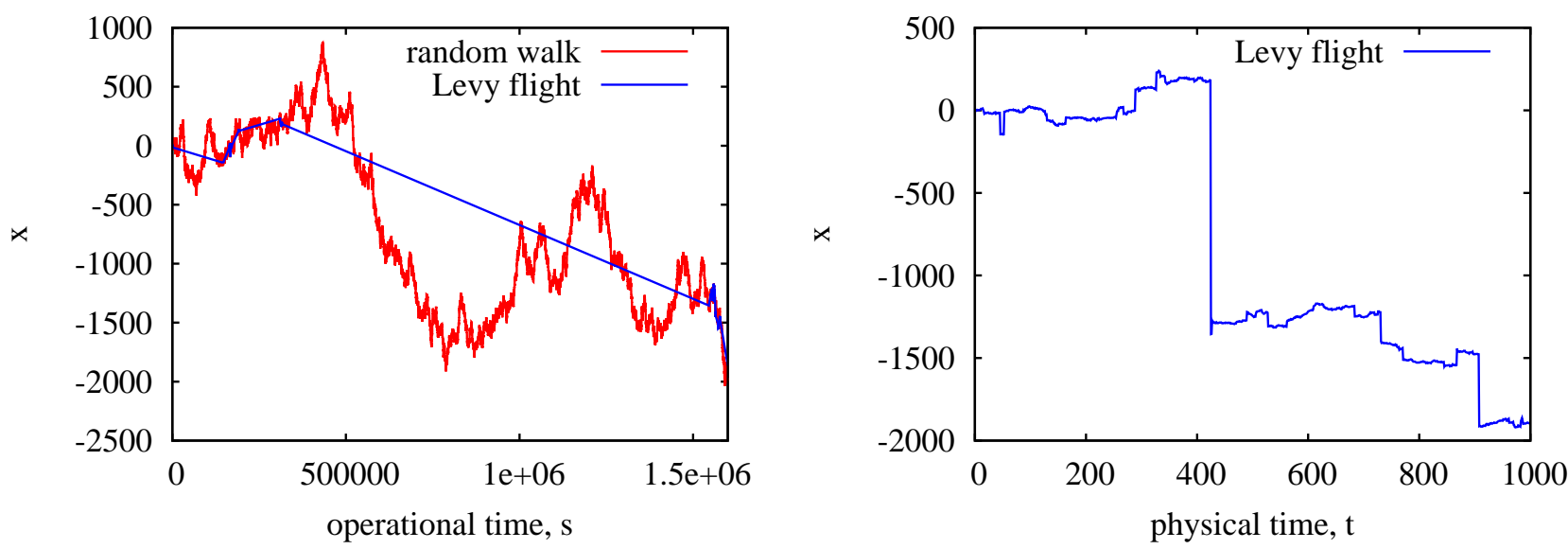

Figure 4.6: Lévy flight subordinated to a random walk. Left panel: The blue line connects the points obtained by irregularly sampling a random walk (red) in operational time s. Right panel: When the irregularly sampled points are transformed into the physical time by "coarse-graining" (removal of the excess, non-sampled points of the random walk trajectory), a Lévy flight process is obtained.

result in (4.32) it is straight-forward to arrive at the expression

$$
f_{\alpha}\left(x_{m}, T\right)=\int_{0}^{\infty} \mathrm{d} S K_{\alpha / 2}(S, T) \cdot f\left(x_{m}, S\right)=2 \cdot p_{\alpha}\left(x_{m}, T\right) .
$$

However, the form of the maximum excursion $f_{\alpha}\left(x_{m}, T\right)$ obtained in (4.33) does not agree with the numerical simulations (see figure 4.7).

The subordination method yields the correct scaling behaviour for the mean perimeter of the convex hull, but it interestingly overestimates the pre-factor. This discrepancy is due to an underestimation of the maximum distribution near its peak at zero, while the tail behaviour of the maximum distribution is accounted for correctly by the subordination method (see figure 4.7). As mentioned earlier, the subordinated Lévy flight is obtained by sampling points from a Brownian trajectory at irregularly spaced time intervals that are distributed according to a broad-tailed inverse power law. Therefore, the presence of large sampling intervals implies that it is relatively likely that the underlying Brownian motion will make small excursions above zero that will be completely missed by the sampling procedure. Consider figure 4.6 for example. In the left panel, we see that the maximum positive excursion achieved by the simple random walk is double that of the subordinated Lévy flight.

\subsection{Conclusion}

In this chapter we have considered two-dimensional properties of anomalous diffusion processes. Based on the method of subordination we have analytically calculated the mean perimeter 

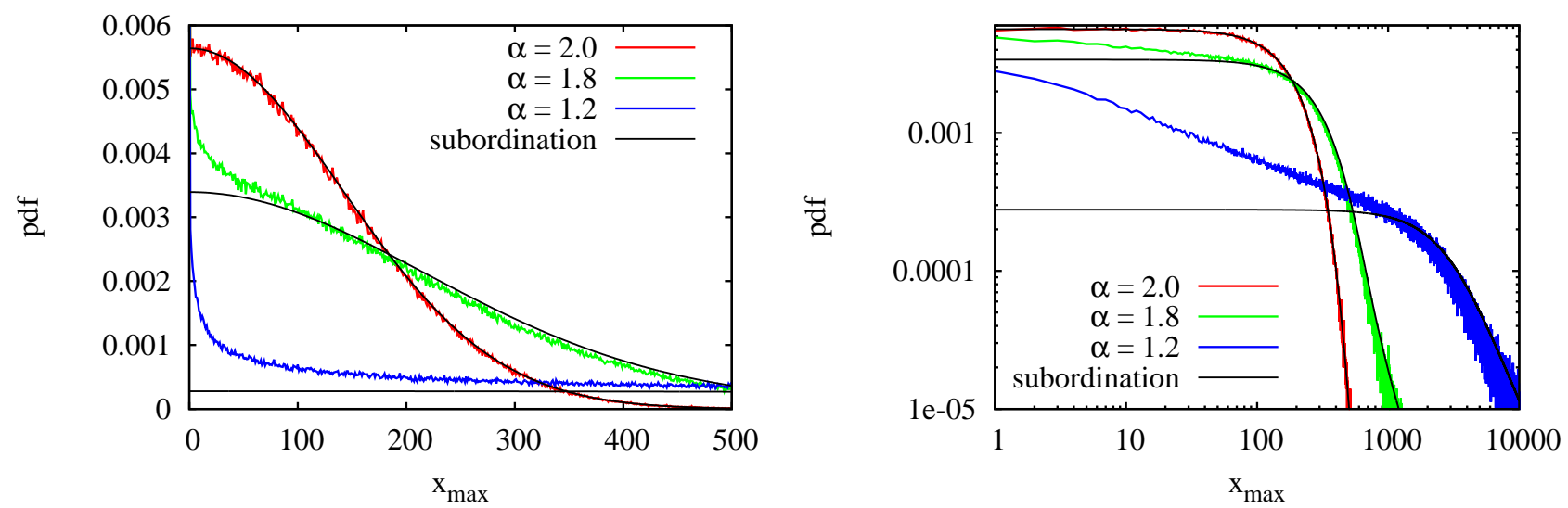

Figure 4.7: Probability distribution of the maximum excursion $x_{m}$ of a Lévy flight. The tree curves were obtained numerically using Lévy flights with different stability parameters, $\alpha=2.0$ (Red), 1.8 (Green) and 1.2 (Blue). The three different colours show the numerical result and the the black lines correspond to the subordinated version obtained from equation (4.33).

and average area of the convex hull for a class of non-Markovian processes. The analytical results were found to agree perfectly with numerical simulations. For the mean perimeter, we generalised our results to the case of subordinated Lévy Flights. Thus for the first time we obtained two-dimensional geometric properties of CTRW processes. We were also able to determine the exact expression for the distribution of the maximum excursion of the CTRW using subordination theory.

Keeping in mind the broad range of disciplines, where the CTRW is employed as a stochastic model, our findings are valuable whenever information about the area or the perimeter of such a two-dimensional process is of interest. 


\section{Summary \& Outlook}

In this dissertation we studied the geometric properties of anomalous diffusion processes.

We have developed a method based on properties of the convex hull that discriminates between normal diffusive processes and super-diffusive processes, based only on the points visited by a random walker. Since time ordering of the data is not required, our method is well suited for the analysis of trajectories in cases where time-ordered data collection is not possible, such as in the standard method for home range measurements. An important next step is to analyse real ecological data, with the goal to contribute to the ongoing debate regarding whether or not certain foraging animals truly perform a Levy walk. Future work should establish methods for hypothesis testing based on the method of convex hull discrimination. This remains challenging since even in the simplest of cases the analytical expression of the required probability distribution is unknown.

The study of convex hulls is important in the context of geometric properties of random processes. We have given two contributions in this regard. First, we have presented some new insights regarding the shape of the distribution of the perimeter and area of convex hulls of random walks. This goes beyond the usual considerations of average properties of convex hulls. In particular, we have used statistical properties of the waiting times between successive hull increments to illustrate why the probability distribution of the perimeter does not satisfy the central limit theorem. Second, by using the concept of subordination we determined the exact analytical expressions for the average perimeter and area of the convex hulls of a class of nonMarkovian processes, namely continuous time random walks. Where possible, we generalised our results to Levy flights. We also use the concept of subordination to develop an alternative and simpler approach to calculating the maximum excursion distribution of a continuous time random walk.

Taken as a whole, these results demonstrate the diversity of approaches that must be attempted when treating non-Markovian, stochastic processes, which require a treatment that lies at the very edge of our knowledge. Since this edge pushes up against reality, the contributions in this thesis should be taken as an indication that, while challenging, theoretical approaches can nevertheless aid us in better understanding real stochastic processes in complex environments. 


\section{Bibliography}

[1] Burt W H (1943) Journal of Mammalogy 24346

[2] Powell R A and Mitchell M S (2012) Journal of Mammalogy 93948

[3] Berg H C (2004) E. Coli in Motion (Springer, New York)

[4] Viswanathan G M, da Luz M G E, Raposo E P and Stanley H G (2011) The Physics of Foraging: An Introduction to Random Searches and Biological Encounters (Cambridge University Press)

[5] Codling E A, Plank M J and Benhamou S (2008) J. R. Soc. Interface 5813

[6] Bartumeus F, da Luz M G E, Viswanathan G M and Catalan J (2005) Ecology 863078

[7] Shlesinger M F and Klafter J (1986) Lévy walks versus Lévy flights in On Growth and Forms NATO ASI Series Volume 100, 1985, pp 279-283 (Martinus Nijhoff Publishers, Amsterdam)

[8] Benhamou S (2007) Ecology 881962

[9] Codling E A and Plank M J (2011) Theor. Ecol. 4397

[10] Edwards A M, Philips R A, Watkins N W, Freeman M P, Murphy E J, Afanasyev V, Buldyrev S V, da Luz M G E, Raposo E P, Stanley H E and Viswanathan G M (2007) Nature 4491044

[11] Edwards A M (2008) J. Animal Ecology 771212

[12] White E P, Enquist B J and Green J L (2008) Ecology 89905

[13] Majumdar S N, Comtet A and Randon-Furling J (2010) J. Stat. Phys. 138955

[14] Boyle S A, Lourenço W C, da Silva L R and Smith A T (2009) Folia Primatol 8033

[15] Mandelbrot B B (1982) The Fractal Geometry of Nature (San Francisco: Feeman)

[16] Geisel T, Nierwetberg J and Zacherl A (1985) Phys. Rev. Lett. 54616 
[17] Cole B J (1995) Animal Behaviour 501317

[18] Viswanathan G M, Afanasyev V, Buldyrev S V, Murphy E J, Prince P A and Stanley H E (1996) Nature 381413

[19] Sims D W et al. (2008) Nature 4511098

[20] N. E. Humphries et al. (2010) Nature 4651066

[21] Ramos-Fernández G, Mateos J L, Miramontes O, Cocho G, Larralde H and Ayala-Orozco B (2004) Beh. Ecol. Sociobiol. 55223

[22] Bénichou O, Loverdo C, Moreau M and Voituriez R (2011) Rev. Mod. Phys. 8381.

[23] Berkolaiko G, Havlin S, Larralde H and Weiss G H (1996) Phys. Rev. E 535774

[24] Berkolaiko G and Havlin S (1997) Phys. Rev. E 551395

[25] G M Viswanathan et al. (1999) Nature 401911

[26] Santos M C, Raposo E P, Viswanathan G M and da Luz M G E (2004) Europhys. Lett 67 734

[27] Shlesinger M F (2007) Nature 45040

[28] Tammero L F and Dickinson M H (2002) The Journal of Experimental Biology 205327

[29] Reynolds A M and Frye M A (2007) PLOS ONE 2(4) e354

[30] Wade A R and Xu C (2013) Arxiv math.PR 1301.4059v1

[31] Clemmons J R and Buchholz R (1997) Behavioral Approaches to Conservation in the Wild (Clemmons J R, Buchholz, eds.) Linking Conservation and Behavior pp 3-22 (Cambridge, Cambridge University Press)

[32] Caro T (1998) Behavioral Ecology and Conservation Biology (Caro T, ed.) The significance of behavioral ecology for conservation biology pp 3-26 (New York, Oxford University Press)

[33] Murphy D D, and Noon B R (1992) Ecol. Appl. 23

[34] Powell R A (2000) Research Techniques in Animal Ecology (Boitani L, Fuller T K, eds.) Animal home ranges and territories and home range estimators pp 65-110 (New York, Columbia University Press)

[35] Börger L, Dalziel B D and Fryxell J M (2008) Ecology Lett. 11637

[36] Dumonteil E, Majumdar S N, Rosso, A and Zoia A (2013) Proc. Nat. Acad. Sci. 1104239 
[37] Randon-Furling J, Majumdar S N and Comtet A (2009) Phys. Rev. Lett. 103140602

[38] Weiss G H and Rubin R J (1976) J. Stat. Phys. 14333

[39] Graham R L (1972) Info. Processing Lett. 1132

[40] Barber C B, Dobkin D P and Huhdanpaa H (1996) ACM Transactions on Mathematical Software 22469

[41] Takács L (1980) Amer. Math. Month. 8742

[42] El Bachir M (1983) L'enveloppe convexe du mouvement brownien Ph.D thesis, Université Paul Sabatier, Toulouse, France

[43] Spitzer F and Widom H (1961) Proc. Amer. Math. Soc. 117506

[44] Redner S (2001) A Guide to First-Passage Processes (Cambridge University Press)

[45] Daniels H E (1941) Proc. Camb. Phil. Soc. 37244

[46] Rubin R J (1972) J. Chem. Phys. 565747

[47] Kuhn W (1934) Koll. Z. 682

[48] Rudnick J and Gaspari G (1987) Science 237384

[49] Weiss G H and Weissman H (1988) J. Stat. Phys. 52287

[50] Klafter J and Sokolov I M (2011) First Steps in Random Walks: From Tools to Applications (Oxford University Press)

[51] Ebeling W and Sokolov I M (2005) Statistical Thermodynamics and Stochastic Theory of Nonlinear Systems Far from Equilibrium (World Scientific Publishing Company)

[52] Allegrini P, Bellazzini J, Bramanti G, Ignaccolo M, Grigolini P and Yang J (2002) Phys. Rev. E. 66 015101(R)

[53] Sims D W, Righton D and Pitchford J W (2007) J. Animal Ecology 76222

[54] Edwards A M et al. (2007) Nature 4491044

[55] Newman M E J (2005) Contemporary Physics 46323

[56] Viswanathan G M, Raposo E P, Bartumeus F, Catalan J and da Luz M G E (2005) Phys. Rev. E 72011111

[57] Plank M J and Codling E A (2009) Ecology 903546

[58] Calenge C, Dray S and Royer-Carenzi M (2009) Ecological Informatics 434 
[59] Metzler R and Klafter J (2000) Phys. Rep. 3391.

[60] Weiss G H (1994) Aspects and Applications of the Random Walk (North Holland)

[61] Fedotov S and Iomin A (2007) Phys. Rev. Lett. 98118101

[62] Olla P (2012) Phys. Rev. E 85021125

[63] Sahimi M (2012) Phys. Rev. E 85016316

[64] Berkowitz B, Cortis A, Dentz M and Scher H (2006) Rev. Geophys. 44 RG2003

[65] Luković M, Geisel T and Eule S (2013) New J. Phys. 15063034

[66] Kramer D L and McLaughlin R L (2001) Amer. Zool. 41137

[67] O’Brien W J, Browman H I and Evans B I (1990) Amer. Scient. 78152

[68] Bartumeus F, Giuggioli L, Louzao M, Bretagnolle V, Oro D and Levin S A (2010) Current Biology 20215

[69] Gorenflo R, Mainardi F and Vivoli A (2007) Chaos, Solitons and Fractals 3487

[70] Fogedby H C (1994) Phys. Rev. E 501657

[71] Barkai E and Silbey R J (2000) J. Phys. Chem. B 1043866

[72] Penson K A and Górska K (2010) Phys. Rev. Lett. 105210604

[73] Sokolov I M (2002) Phys. Rev. E 66041101

[74] Metzler R and Klafter J (2004) J. Phys. A: Math. Gen. 37 R161

[75] Feller W (1968) An Introduction to Probability Theory and Its Applications vol 1 (John Wiley \& Sons)

[76] Schehr G and Le Doussal P (2010) J. Stat. Mech. P01009

[77] Carmi S, Turgeman L and Barkai E (2010) J. Stat. Phys. 1411071

[78] Comtet A and Majumdar S N (2005) J. Stat. Mech. P06013

[79] Franke J and Majumdar S N (2012) J. Stat. Mech. P05024

[80] Sokolov I M (2000) Phys. Rev. E 63011104

[81] Brockmann D and Sokolov I M (2002) Chemical Physics 284409 


\section{Acknowledgements}

First of all I would like to thank Theo Geisel for his support in every respect and his supervision during the fantastic time I had since Elena and I moved to Göttingen. The scientific environment could not be better.

I also thank the entire thesis committee (Theo Geisel, Marc Timme and Fred Wolf) for their guidance and the time they devoted to me during my $\mathrm{PhD}$ studies.

Second I would like to thank Stephan Eule for his supervision, patience and advice. I also would like to thank Jan Nagler for countless scientific suggestions and inspiring ideas that greatly helped me to advance things in many respects. Equally, uncountable many thanks go to Marc Timme for his open-mindedness, support, positivity and for always strongly constructive feedback.

I am thankful and grateful for the invaluable discussions I had with Max Puelma Touzel, David Hofmann, Mario Modena and Humberto Sandmann.

Grazie infinite!

I thank Elena, my love, for her understanding and patience in stressful times and everything she did to support me. Not only for good times but also for the naked survival is it necessary to have friends and family around. I am deeply indebted to them and hope that some day I can return at least a little. 


\section{Curriculum Vitae}

Name: Mirko Luković

Place and date of birth: Belgrade, $9^{\text {th }}$ July, 1981.

\section{Education}

Ph.D. Degree in Physics obtained from the Georg-August-Universität, Göttingen Graduate School for Neurosciences, Biophysics, and Molecular Biosciences (GGNB) and the International Max Planck Research School (IMPRS).

Defence: February 6, 2014.

Thesis: "Anomalous Diffusion in Ecology".

Key words: Convex Hulls, Non-Poissonian systems, inverse power law distributions, Lévy walk, continuous time random walks, subordination.

Adviser: Prof. Dr. Theo Geisel.

Master's Degree in Solid-State Physics obtained from the University of Pisa.

Defence: May 26, 2008.

Thesis: "Towards a Theory of Complexity Matching".

Key words: Non-Poisson systems, inverse power law distributions, renewal events, beyond stochastic resonance, definition of a complex system, perennial aging, $1 / f$-noise, ergodicity breakdown.

Adviser: Prof. Paolo Grigolini.

Bachelor's Degree in Physics obtained from the University of Pisa.

Defence: September 17, 2004.

Thesis: "Some Applications of Group Theory to Elementary Quantum Mechanics".

Adviser: Prof. Emilio D'Emilio

\section{Publications Related to the Dissertation}

Area and perimeter covered by anomalous diffusion processes - M. Luković, T. Geisel and S.

Eule, New J. Phys. 15063034 (2013). 